Portland State University

PDXScholar

Winter 4-6-2018

\title{
Health Risks in Medical Homes and their Effects on Emergency Department and Inpatient Expenditures: a Focus on Patient-Centered Primary Care Homes in Oregon
}

Kweku Nyameyepa Wilson

Portland State University

Follow this and additional works at: https://pdxscholar.library.pdx.edu/open_access_etds

Part of the Medicine and Health Sciences Commons

Let us know how access to this document benefits you.

\section{Recommended Citation}

Wilson, Kweku Nyameyepa, "Health Risks in Medical Homes and their Effects on Emergency Department and Inpatient Expenditures: a Focus on Patient-Centered Primary Care Homes in Oregon" (2018). Dissertations and Theses. Paper 4254.

https://doi.org/10.15760/etd.6138

This Dissertation is brought to you for free and open access. It has been accepted for inclusion in Dissertations and Theses by an authorized administrator of PDXScholar. Please contact us if we can make this document more accessible: pdxscholar@pdx.edu. 
Health Risks in Medical Homes and Their Effects on Emergency Department and Inpatient Expenditures:

A Focus on Patient-Centered Primary Care Homes in Oregon

by

Kweku Nyameyepa Wilson

A dissertation submitted in partial fulfillment of the requirements for the degree of

Doctor of Philosophy

in

Health Systems and Policy

Dissertation Committee:

Neal T. Wallace, Chair

Jill Rissi

Ana Quiñones

Matthew Carlson

Portland State University

2018 
(C) 2018 Kweku Nyameyepa Wilson 


\begin{abstract}
The fragmented approaches to delivering health care services in the United States, along with the associated structural inefficiencies and unsustainable increases in health care costs affecting all payers, compel the need for reform. Various federal and state-level delivery system reform models have emerged in response.

The Medical Home (MH) is one of such reform models. In 2004 a national initiative entitled "The Future for Family Medicine Project" identified the lack of emphasis on comprehensive primary care, especially for chronic care patients, and proposed the introduction of MHs to improve comprehensive primary care delivery for every patient. Oregon's MH variant, the Patient-Centered Primary Care Home (PCPCH), was introduced in 2009 as part of a state-wide health reform initiative ushered in by the passage of House Bill 2009 to promote the Triple Aim.

Since 2011, over 600 primary care clinics have been recognized as PCPCHs. Proponents of the model argued that it will help improve comprehensive primary care services upstream and reduce inappropriate utilization of Emergency Department (ED) and Inpatient (IP) care and expenditures downstream. Evidence on the model's application to reduce ED and IP utilization and expenditures have so far been mixed (Friedberg et al., 2014; Jackson \& Williams, 2015; Baseman et al., 2016). Based on growing interests in the effects of the model's application to provide care for different types of patients, this research was designed to evaluate the policy effects of the application of PCPCHs, with a focus on PCPCHs that treat greater proportions of chronic care patients, to answer the following questions:
\end{abstract}


(1) What is the average chronic disease burden of PCPCHs, and how does their average chronic disease burden compare to the communities PCPCHs are in pre-post PCPCH recognition?

(2) How do primary care expenditures change based on the chronic disease burden of PCPCHs?

(3) Do PCPCHs that engage more high chronic disease burden patients have more reductions in ED and IP expenditures?

For this research, a chronic disease burden measure was developed from 10 markers of chronic conditions. This measure was then used to stratify PCPCH clinics and their comparators into high and low chronic disease burden clinics. The research was designed as a natural experiment, utilizing difference-in-difference methods to measure outcome differences pre-post $\mathrm{PCPCH}$ policy implementation and comparing outcome differences between PCPCHs and their control groups. The unit of analysis was PCPCH clinics. The theoretical perspectives that informed this research were Risk Selection and Complex Adaptive Systems (CAS). Data from Oregon's All Payer All Claims (APAC) data system, which included 16 quarters of claims and eligibility data from fourth quarter 2010 to third quarter 2014, as well as $\mathrm{PCPCH}$ attestation data on 525 clinics were utilized for this research.

The results suggest that the chronic disease burden for PCPCHs was significantly lower than their comparator groups before clinics recognition as PCPCHs, but the chronic disease burden did not change after clinics recognition as PCPCHs. Average primary care expenditures did not change after PCPCH 
recognition. Average ED and average IP expenditures for high chronic disease burden PCPCHs did not change but rather decreased significantly for low chronic disease burden PCPCHs.

The results imply that the distribution of chronic disease burden in PCPCHs is important and related to ED and IP expenditures, but in a different direction than expected. The results also suggest that focusing on low chronic disease burden patients in PCPCHs could help reduce ED and IP expenditures in the short and medium terms. Policies to engage a broader mix of chronic disease burden patients in PCPCHs could help increase savings from ED and IP utilization. The results also suggest the need for more research to improve current understanding of how PCPCHs are impacting health care trajectories in the current delivery system environment. 


\section{Dedication}

I dedicate this dissertation to both Mrs. Wilsons in my life: my dear mother, Emelia Araba Wilson, and my dear wife, Diana Ameyo Wilson. I could not have done this without your continuous love and encouragement. This dissertation is also dedicated to the blessed memory of John Charles Wilson my father, Ralph Hull, and Rex and Carole Parnell, I miss you all and wish you were here to share this moment with me. You touched my life in many ways, and I could not have done this without your help and support. Thank you. 


\section{Acknowledgements}

To God be the glory for this accomplishment, which means so much to me and my family. The journey to complete my doctoral dissertation was long but stimulating and fulfilling. I heard from many who have travelled this path that pursuing a Ph.D. is a quest to become "a knowledge creator." It is my hope that the conclusion of this research marks the beginning of the next journey: to use the knowledge I have acquired to help advance the cause for access to affordable and quality health care services.

I stood on many broad and stable shoulders to arrive at this stage and could not have completed this research without support from my unwavering dissertation committee, my family, and many people from all walks of life. I therefore agree with Whitehead's observation that "No one who achieves success does so without the help of others. The wise and confident acknowledge this help with gratitude." With that in mind, I would like to express my sincerest gratitude to several very special people. To my dissertation committee members, I am thankful for your time, advice, and many contributions to this research. Most importantly, to Dr. Neal Wallace, my chair and mentor, you are everything I hoped for and more. I will forever be grateful for your invaluable support for my dissertation and your friendship. I also look forward to the opportunity of working with you as both my mentor and friend on many research endeavors. To Dr. Sherril Gelmon, I cannot find words to express my gratitude for your encouragement, and many contributions towards the completion of my dissertation. I could not have completed this research without your invaluable 
support. I am also impressed with the work you and Neal, and with the support of many faculty members, have invested in developing the Health Systems and Policy program. I have no doubt that the program will grow and receive national recognition under your esteemed leadership. To Drs. Jill Rissi, Ana Quiñones, and Matthew Carlson, I am grateful and happy that you agreed to work with me on this dissertation. Thank you.

To my dear mother Emelia Araba Wilson, your son got it done! You have always been my biggest cheerleader, the values you taught me have brought me this far and I am very grateful for all your sacrifices for me. Thank you. To Diana Ameyo Wilson, my dear wife and best friend, thank you for your friendship, many sacrifices, and meaningful support for me throughout this journey. I could not have done this without your unconditional support. Thank you. To my brothers and sisters, you mean so much to me. Thank you for inspiring me to get this far. To Wilma Lee Hull, you are my angel, and I am deeply grateful for your friendship, timely and countless acts of kindness for me and my family for which I will forever be grateful. Thank you. To my dear sister Patty Parnell, Dr. Vivian Djokotoe, Hilda Djokotoe and Ernestina Djokotoe, I am grateful for all your love, help, and support throughout this journey. Thank you.

I must confess that while I looked forward to writing my acknowledgements, the actual writing unleashed a range of mixed emotions. From the joy and realization that this chapter of my life is concluding successfully, concerns about the bonds and friendships I leave behind at Portland State University, to the cherished memories that 
will be with me forever. To my colleagues and friends still in the Health Systems and Policy program, you are in good hands, I wish you all the best and hope our paths cross many more times. To the faculty and staff of the Hatfield School of Government, thank you for your contributions to my academic success. To all who have been helpful to me along this journey but were not mentioned individually in my acknowledgements because of time and space constraints, thank you for your help and may God richly bless you all. 


\section{Table of Contents}

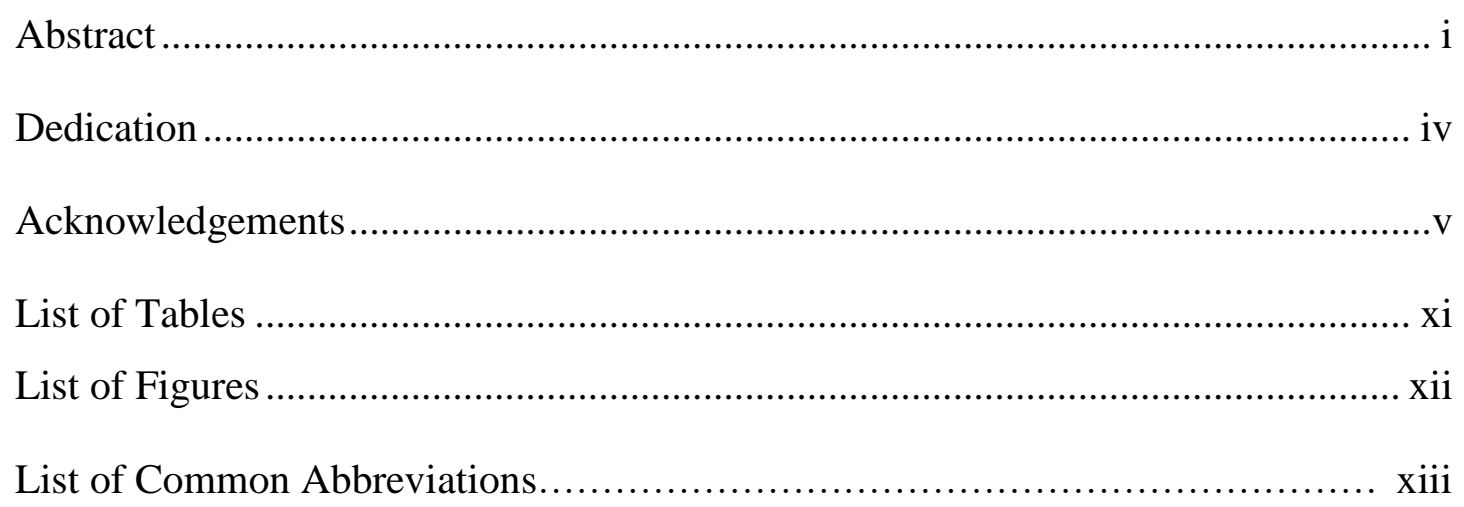

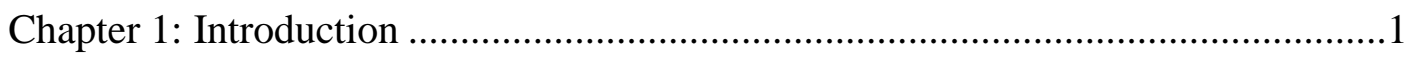

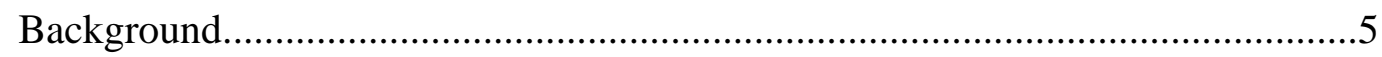

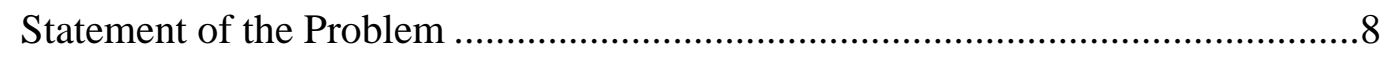

Purpose and Significance of the Study ....................................................... 10

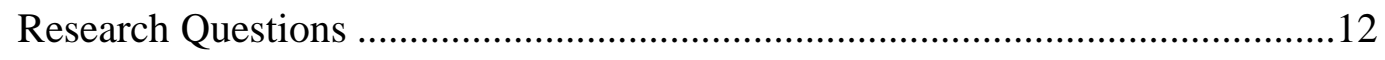

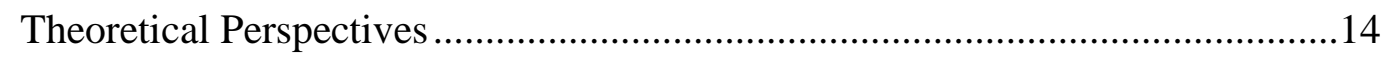

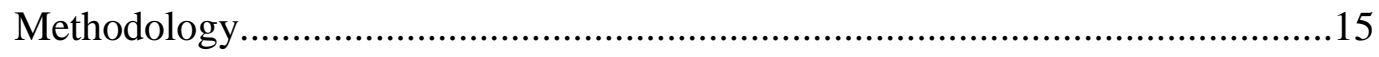

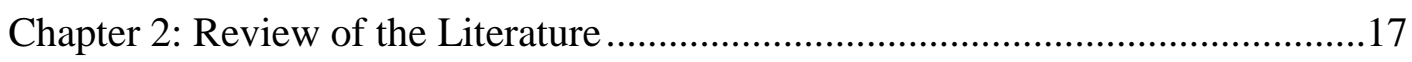

History of the Medical Home Model.............................................................17

The Patient-Centered Medical Home (PCMH) Model.....................................20

Oregon's Patient-Centered Primary Care Home (PCPCH) Model ......................25

PCPCH and other Medical Home Models..................................... 27

PCPCH and other State's Medical Home Programs.......................... 28

Evidence of Improvements in Cost-Sensitive Outcomes of Care................ 33

The Cost-Sensitive Benefits of Primary Care............................... 34

The Cost-Sensitive Outcomes from Chronic Disease Management..............38 
Improved Cost-Sensitive Outcomes from Pediatric Populations.............. 43

Improved Cost-Sensitive Outcomes from Adult Populations................ 44

Improved Cost-Sensitive Outcomes from Integrated Delivery Systems (IDS)...46

Improved Cost-Sensitive Outcomes from State Programs ................................48

The Importance of Health Risks to this Research ...........................................53

Association between Health Risks and Outcomes in Medical Homes .................55

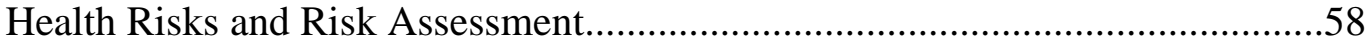

Theoretical Perspectives .........................................................................6

Grand Summary of the Evidence ...........................................................67

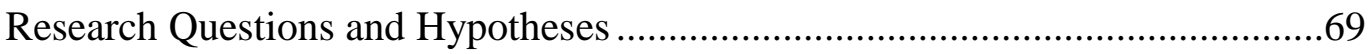

Chapter 3: Research Methods ..................................................................... 71

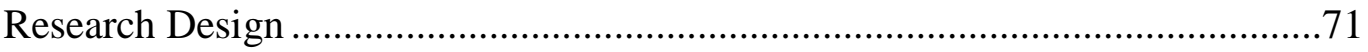

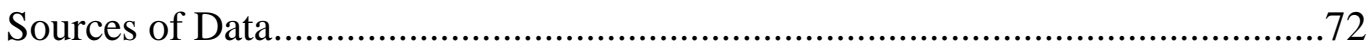

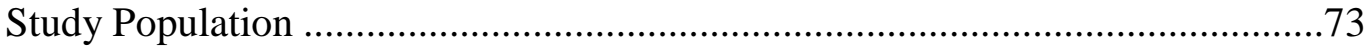

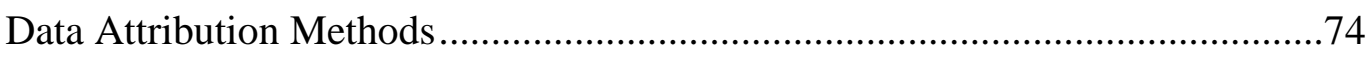

Aggregate PCPCH and Comparator Measures..................................................76

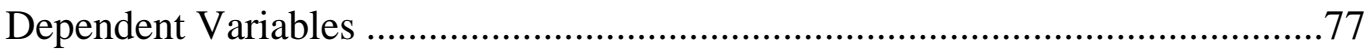

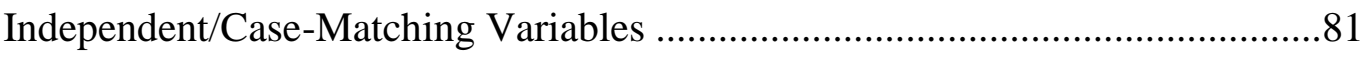

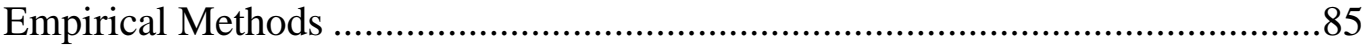

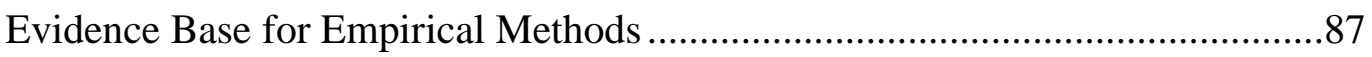

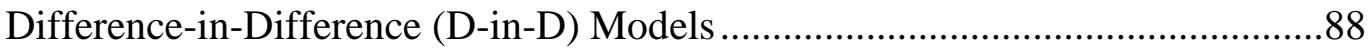

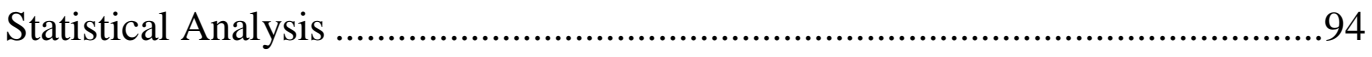




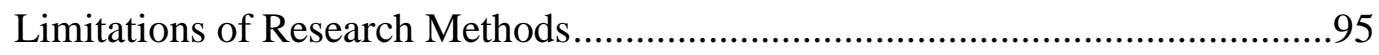

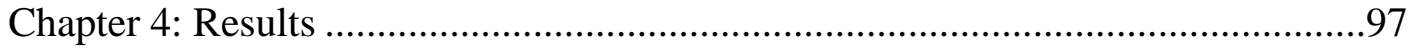

Summary of Data Analysis.................................................................... 97

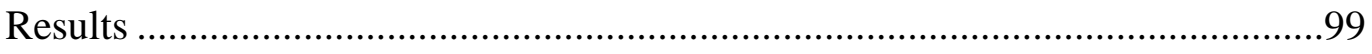

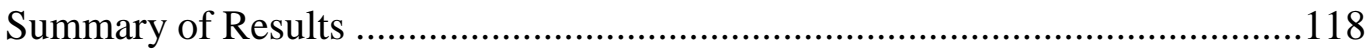

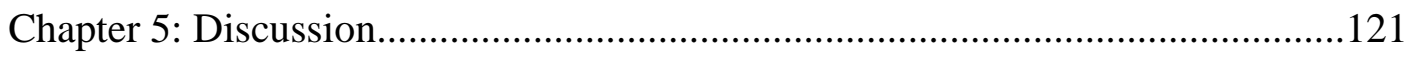

Chronic Disease Burden Levels in PCPCHs .................................................123

Primary Care Expenditures in PCPCHs ..................................................... 126

Emergency Department and Inpatient Expenditures in PCPCHs......................128

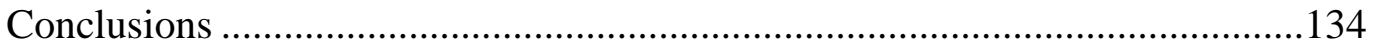

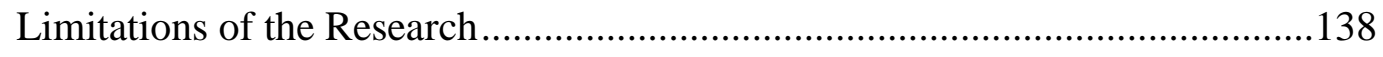

Implications for Policy Development ........................................................141

Recommendations for Further Research ................................................. 145

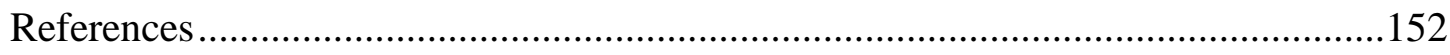




\section{List of Tables}

Table 2.1: Primary Differences between Patient-Centered Medical Homes and Health

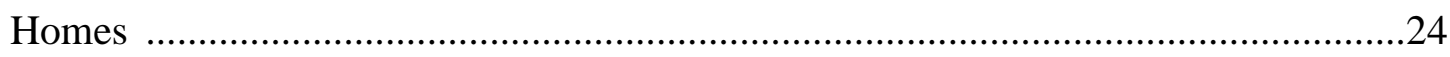

Table 2.2: Primary Standards for Patient-Centered Medical Home Certification ......26 Table 4.1: Patient Characteristics in PCPCHs and Their Community Comparators 101 Table 4.2: Average Changes in Chronic Disease Burden by PCPCHs .....................102 Table 4.3: Patient Characteristics in High and Low Chronic Disease Burden PCPCHs 105

Table 4.4 Average Changes in Primary Care Spending by PCPCHs .......................107 Table 4.5: Average Changes in Emergency Department Spending by PCPCHs .....110 Table 4.6: Average Changes in Inpatient Spending by PCPCHs

Table 4.7: Average Changes in Emergency Department and Inpatient Spending by PCPCHs

Table 4.8: Average Changes in Emergency Department, Inpatient, and Primary Care Spending by PCPCHs .118 


\section{List of Figures}

Figure 2.1: Total Health Expenditures for $2012=1.35$ Trillion .................. 57

Figure 3.1: Social Determinants of Health \& Equity............................ 84 


\section{List of Common Abbreviations}

AAP American Academy of Pediatrics

ACSC Ambulatory Care Sensitive Conditions

AHRQ Agency for Healthcare Research and Quality

APAC All Payer All Claims Data Systems

BPH Blueprint for Health

CAS Complex Adaptive Systems

CCM Chronic Care Model

CCO Coordinated Care Organizations

CCNC Community Care of North Carolina

CDMC Chronic Disease Management Companies

CHIP Children's Health Insurance Program

CMS Center for Medicare and Medicaid Services

CPCI Comprehensive Primary Care Initiative

CSHCN Children with Special Health Care Needs

D-in-D Difference in Difference

ED Emergency Department Care

FFS Fee-For-Service

GHC Group Health Cooperative

GHS Geisinger Health Systems

HB House Bill

HH Health Homes

HIT Health Information Technology

HMO Health Maintenance Organization

ICD International Statistical Classification of Diseases and Related Health Problems

IDS Integrated Delivery Systems 
IOM Institute of Medicine

IP Inpatient Care

MEdpAC Medicare Payment Advisory Commission

MH Medical Homes

MHCP Medical Home Certification Process

MHI Medical Home Index

NCQA National Committee for Quality Assurance

OHA Oregon Health Authority

OHP Oregon Health Plan

PACT Patient Aligned Care Team

PC Primary Care

PCP Primary Care Practitioner

PCMH Patient-Centered Medical Home

PCPCH Patient-Centered Primary Care Homes

PCMHI Patient-Centered Medical Home Initiative

PHN Proven Health Navigator

PPACA Patient Protection and Affordable Care Act

PCPCC Patient-Centered Primary Care Collaborative

QALY Quality Adjusted Life Years 
Health Risks in Medical Homes and Their Effects on Emergency Department and Inpatient Expenditures: A Focus on Patient-Centered Primary Care Homes in Oregon

\section{Chapter 1: Introduction}

"Health care today is marked by structural inefficiencies, unprecedented costs, and fragmented care delivery, all of which place increasing pressure and burden on individuals and families, providers, businesses, and entire communities. The consequent health shortfalls are experienced across the whole population, but disproportionately impact our most vulnerable citizens due to their complex health and social circumstances" (Dzau et al., 2017).

While the effects of the above-mentioned problems on health care delivery systems and outcomes have become almost passé, their direct and indirect impacts compel reform to improve the organization, delivery, and payments for health care services. Among the proposed reform models for improving the delivery and outcomes of health care is the Medical Home (MH) model. This model focuses on comprehensive primary care and the use of provider teams for coordinating and integrating care continuously for all patients. This approach to care is expected to improve comprehensive primary care upstream, especially for patients with chronic physical and behavioral health conditions, thereby helping reduce downstream demand for Emergency Department (ED) and Inpatient care (IP) utilization and expenditures.

An environmental scan of the burden of chronic care in this country suggests that the MH model's approach to delivering care can be helpful: About 133 million 
people, including 10 percent of children and adolescents, are living with a minimum of one chronic health condition (Kaiser Family Foundation [KFF], 2012, p. 6). In Oregon, about 27 percent of the non-elderly population have chronic conditions (Claxton, Cox, Damico, Levitt, \& Pollitz, 2016). Chronic care is a significant driver of health care expenditures because it manifests in disproportionate utilization of health care resources. It is estimated that 84 percent of health care spending is driven by patients with chronic conditions (Davis, Schoen, \& Stremikis, 2010; Bipartisan Policy Center [BPC], 2012). About 5 percent of the population with complex chronic medical conditions account for 50 percent of total health care expenditures (Anderson, 2010).

The Chronic Care Model (CCM) was introduced to help manage care for such patients, and while some research suggests that the introduction of the CCM has improved the management of chronic conditions (Wagner et al., 2001; Coleman, Austin, Brach, \& Wagner, 2009), the country's changing demographic structure and the association between aging and chronic health conditions suggest that the burden of chronic care and its associated costs may continue to rise without support for delivery models that emphasize upstream care through comprehensive primary care, care coordination and integration, and the alignment of value-based payment systems to incentivize such care as well as improve outcomes.

MHs and their variants are reform models that continue to gain national attention and support for delivering such improvement in care and outcomes. In Oregon, this model is referred to as the Patient-Centered Primary Care Home 
(PCPCH) model. At their core, PCPCHs emphasize the integration of comprehensive primary care into health care delivery. Conceptually, the model is designed to deliver patient-and family-centered care through continuously coordinating and integrating care, along with value-based payments to incentivize integration across provider teams (Rittenhouse, Thom, \& Schmittdiel, 2010; Starfield, 2011). Proponents of MH's argue that such care will be beneficial for all patients, especially chronic physical and behavioral health condition patients who need comprehensive primary care through care coordination, integration, and continuity through relationships with provider teams. Such upstream care is expected to reduce downstream utilization of ED, IP care, and expenditures (Berwick, Nolan, \& Whittington, 2008; Medicare Payment Advisory Commission [MEdpAC], 2008; Oregon Health Authority [OHA], 2010; Peikes, Dale, Lundquist, Genevro, \& Meyers, 2011).

The American Academy of Pediatrics (AAP) and the Institute of Medicine (IOM) consider the model transformational for the delivery of quality patientcentered care: "Ultimately, system-wide changes will need to ensure high-quality health care for all Americans; such changes should include taking steps to ensure that every American has a personal MH” (Martin, Avant, \& Bucholtz, 2004, p. S.3-4). Almost all states in the United States currently have varying levels of integration and emphasis on MH's as part of broader health care delivery and payment reforms (Takach, Gauthier, Sims-Kastelein, \& Kaye, 2010). While it can be argued that the MH model in theory can improve chronic care and reduce health care expenditures through increased upstream use of primary care and reduced downstream use of ED 
and IP, the complex health care delivery system environment suggests that the effectiveness of the model must be continuously evaluated to determine whether its application continues to reduce ED and IP utilization and expenditures, and under what conditions such improvements are realized.

The purpose of this research, therefore, is to evaluate the application of the Patient-Centered Primary Care Homes (PCPCH) in Oregon with a focus on the model's application in practices with different mixes and types of chronic condition patients. A chronic disease burden measure ${ }^{1}$ will be developed and used as a proxy health-risk measure for this research, and it will be developed consistent with prior health services literature on proxy health-risk measures (Ash \& Ellis, 2012; Hornbrook \& Goodman,1996; Ash et al., 2000). Individual chronic disease burden will be measured as the sum of 10 markers of chronic conditions. Primary care practice level chronic disease burden will be measured as the average of practices' patient-level chronic disease burden measures. The practice level measure will be used to compare practices within their communities as well as to identify PCPCH practices with higher versus lower average patient chronic disease burden. This evaluation will specifically determine the relative effectiveness of the application of PCPCHs, based on the average chronic disease burden of PCPCH practices, the average primary care expenditures by chronic disease burden levels (high versus low)

\footnotetext{
${ }^{1}$ The Centers for Disease Control and Prevention define chronic diseases as diseases that are prolonged and not spontaneously resolved, and for which a complete cure is rarely achieved (CDCP, 2015). For this research, the diagnosis codes for 10 of such patient conditions are aggregated and weighted to construct a chronic disease burden measure. The average chronic disease burden, therefore, refers to the average of the 10 chronic conditions.
} 
in PCPCHs, and the average ED and average IP expenditures by chronic disease burden levels (high versus low) of PCPCHs. The results of this research will contribute to the broad-based evidence on the different implementations of the model, and relative effectiveness of the model's application to reduce ED and IP expenditures, especially for chronic care patients.

\section{Background}

Cognizant of the unsustainable upward trajectory of health care costs as well as the direct and indirect system pressures on state budget priorities, Oregon embarked on a health system reform initiative to transform the organization, delivery, and payment for health care services. Among the policy recommendations of the state's health care reform law (HB 2009) were emphasizing comprehensive primary care and expanding access to primary care services through the PCPCH model, and the use of coordinated care organizations (CCOs) as delivery platforms for integrating care for all patients, including those in the Oregon Health Plan. These reforms were designed to help promote the state's Triple Aim: improved patient's experience of care, reduced per capita costs, and improved health of the state's population (OHA, 2010).

Prior to HB 2009, the landscape of health care needs in the state suggested that more than 1.5 million people (61 percent of adults) had at least one of the following chronic conditions: arthritis, asthma, diabetes, heart disease, high blood pressure, high cholesterol, or stroke. In addition, about 89 percent of Oregonian adults had at least one of the following risk factors for the development of additional chronic 
conditions: current smoker; overweight or obese; or physically inactive (OHA, 2012). Over $\$ 2.2$ billion annually was spent on hospitalization for chronic care related health issues, some of which for Ambulatory Care Sensitive Conditions (ACSC) (OHA, 2012). The growing number of patients with chronic physical and behavioral health conditions suggested that coordinating and integrating primary care services in PCPCHs could reduce downstream utilization, expenditures, and overall health care costs.

The recognition of PCPCHs in Oregon began in October 2011; over 600 PCPCH clinics and providers have since been recognized as PCPCHs by the state (OHA, 2012a). This model is being implemented as part of an integrated but stepwise plan to promote the state's Triple Aim. The momentum for implementing PCPCHs in Oregon was accelerated at the federal level by the passage of the Patient Protection and Affordable Care Act (PPACA) in 2010, the Comprehensive Primary Care Initiative (CPCI) in 2011, and the State Innovation Models grant in 2013 (OHA, 2013). These federal initiatives provided funding and technical assistance for Oregon's transformation efforts. Specifically, the PPACA provided incentives under sections 2703 and 3502 for MHs, and for Health Homes for Medicaid recipients who have at least two chronic conditions (PPACA, 2010). The step-wise approach to health system transformation in Oregon culminated in the creation of CCOs to coordinate care across different regions of the state; there are currently 15 recognized CCOs in Oregon. CCOs' capacity to effectively coordinate care at the community level depends on working closely with PCPCHs and other primary care providers. 
Achieving the Triple Aim in Oregon will therefore depend on how well CCOs coordinate care across PCPCHs and other provider settings to engage ${ }^{2}$ the state's Medicaid patients, i.e. Oregon Health Plan (OHP) patients, and reduce expenditures associated with the overuse and misuse ${ }^{3}$ of ED and IP.

The available evidence on $\mathrm{PCPCH}$ recognition in the state suggests that primary care clinics that have been recognized as PCPCHs are diverse. The clinics vary based on their payer type, product mix, and geographic location. These differences could influence the patient mix in PCPCH clinics. If we assume that most health care encounters begin with primary care in ambulatory settings such as PCPCHs, then the coordination and integration of care for patients who have chronic conditions in PCPCHs will be effective in improving ED and IP utilization along with their associated health care expenditures. Evaluating the average levels of patient chronic conditions in PCPCHs in comparison to the communities they reside, and their relationships to expected $\mathrm{PCPCH}$ outcomes such as reductions in ED and IP expenditures, will provide important evidence regarding the relative effectiveness of the model's application.

\footnotetext{
${ }^{2}$ Patient engagement broadly refers to the role of patients in their care, practice improvement, policy design and implementation, and communications. For this research, engagement refers to the delivery of appropriate and patient-centered primary care services.

${ }^{3}$ Overuse and misuse are also referred to as inappropriate use in health services literature and in this study. The terms refer to the use of emergency department and inpatient care services by patients whose healthcare needs are non-urgent or could be appropriately treated or managed in primary care settings. This definition recognizes that in some communities and in some instances, the shortage of primary care providers and access to primary care services leaves patients with no options other than the emergency department and inpatient care services.
} 
It can be argued from a systems perspective that coordinating and integrating care in PCPCHs for patients who have chronic physical and behavioral health conditions will be an effective approach to promoting the Triple Aim outcomes in Oregon. While all patient populations can benefit from receiving care in PCPCHs, patients who have various levels of chronic physical and behavioral conditions may have more benefits (Patient-Centered Primary Care Collaborative, 2016; Pikes et al., 2011). The focus of this research on the levels of chronic physical and behavioral health conditions in PCPCH clinics will be important for evaluating this argument.

\section{Statement of the Problem}

Historical evidence suggests that MHs were first applied to the care of children with special health care needs (Sia, Tonniges, Osterhus, \& Toba, 2004; Robert Graham Center, 2007; Epstein, Fiscella, Lesser, \& Stange, 2010). The benefits of the model led to renewed suggestions for its broad application. The mixed evidence for the broad application of the model as part of ongoing delivery system reforms around the country (Gunterman, 2007; Rittenhouse, Thom, \& Schmittdiel, 2010), however, has compelled some researchers to argue for cautious approaches to implementing the model (Fisher, 2008; Kilo \& Wasson, 2010; Pikes et al., 2011). Proponents of this view argue for more research evidence on the model's application, especially for different patient groups in different delivery systems and the environmental impacts of the model's applications. A recent evaluation of the PCPCH model suggested the following among its conclusions: "[T]he analysis indicates that, in most cases while the PCPCH attributes clearly influence cost and 
utilization measures, they rarely do so in ways that are independent and can be attributed to a specific attribute; the cumulative effect of the $\mathrm{PCPCH}$ attributes has more impacts than the independent effects" (Gelmon, Wallace, Sandberg, Petchel, \& Bouranis, 2016, p. 56).

The relationship between health risks and outcomes suggests that higher health risks may be correlated to downstream expenditures through the overuse and misuse of ED and IP (Frank, McGuire, \& Newhouse, 1995; Reinhardt, 1998). The emphasis on MHs is intended to help reduce such overuse and misuse of ED and IP through effective care coordination and integration for patients who have chronic physical and behavioral health conditions. Ascertaining the average chronic disease burden of PCPCHs, along with the relationship between high and low chronic disease burden PCPCHs and reductions in primary care (PC), ED, and IP expenditures, will provide further insights into the relative effectiveness of the model's application.

Evaluating the levels of health risks in PCPCHs is important because the heterogeneity of health risks (Newhouse, 1996a, b) and the market-based approach to providing health care services make risk selection possible in health care services delivery. Risk selection in health care delivery is influenced by a delivery system environment that is characterized by fragmentation in the organization, delivery, and payment systems for health care (Arrow, 1963; Newhouse, 1996a). It can be inferred that the potential benefits of the model's application can be attenuated when providers select risks or when payment incentives are not well aligned for PCPCHs to provide care to patients whose health risks disproportionately drive health care expenditures. 
Risk selection can be positive or adverse when providers choose healthier or sicker patients than the average population risk. Risk selection can be initiated by patients or providers, but while risk selection in $\mathrm{PCPCH}$ has not been studied, the complex and adaptive nature of the delivery system environment as well as the effects of risk selection on outcomes and payment incentives for providers make it imperative to investigate the levels of health risks in PCPCHs. The state-wide implementation of PCPCHs provides opportunities for investigating the relative effectiveness of the model's application in Oregon.

Plesk (2001) argued that changes in the organization of health care delivery occurs "in the zone of complexity and have nonlinear effects" (p.12) because of the interactions among different system elements in the delivery environment. It can be argued, by extension, that the relative effectiveness of the PCPCH model's application to reduce ED and IP expenditures may be affected by this zone of complex interactions. The thesis of this research is that the relative effectiveness of the PCPCH model's application to reduce ED and IP expenditures will depend on engaging patients whose chronic disease burden (chronic physical and behavioral health conditions) disproportionately drive up utilization and expenditures.

\section{Purpose and Significance of the Study}

The main objectives of this relative effectiveness evaluation of PCPCHs are to determine the following:

1. Does the average chronic disease burden in $\mathrm{PCPCH}$ change pre-post PCPCH recognition? 
2. Do primary care expenditures change based on the chronic disease burden of PCPCHs?

3. Do PCPCHs that engage more high chronic disease burden patients have more reductions in ED and IP expenditures?

This research will focus on short-term outcomes because of data concerns. PCPCH recognition in Oregon commenced October 2011; therefore, the availability of data will only permit a short-term study. Focusing on the short term is also important because resource constraints and the competitive health care delivery environment suggest that the transformational path and long-term sustainability of any reforms, including those for PCPCHs, will depend on demonstrable short-term improvements in containing or reducing health care costs. The results of this research will therefore improve existing knowledge on the PCPCH model's application. The emphasis on practices' average chronic disease burden levels, combined with how average chronic disease burden levels affect short-term outcomes that policymakers focus on to determine the relative effectiveness of PCPCHs, will help ascertain whether PCPCHs that engage patients with a higher chronic disease burden achieve more reductions in ED and IP expenditures. This is important because healthy patients may have comparatively lower utilization, while patients who have higher chronic physical and behavioral health conditions may have higher utilization (Davis, Schoen, \& Stremikis, 2010; BPC, 2012).

The evidence of risk selection in health care delivery and the effects of selection on delivery system effectiveness (Frank, McGuire, \& Newhouse, 1995) 
highlight this research's importance. Because differences in chronic disease burden levels may translate into differential utilization and expenditures, economic agents such as health care providers may focus on low chronic disease burden patients. The changing demographics of the state's population and the growing proportion of people living with chronic conditions suggest that reform models that do not engage such patients may not be sustainable or value adding over time. Insights from this research can further help determine whether chronic disease burden levels in PCPCHs are a stable measure for evaluating the relative effectiveness of the model's application.

The results could also shape incentive policies for recognizing and rewarding PCPCHs that engage health risks in proportion to their community presence. Most importantly, this study can help guide state policymakers on whether and under what conditions PCPCHs contribute to reducing ED and IP expenditures. Such evidence will be important for policy decisions because effective system transformation will require simultaneous reforms of delivery processes and payment mechanisms (Davis, 2007; Devers \& Berenson, 2009; Luft, 2009).

\section{Research Questions}

This research proposes that engaging patients who have higher levels of chronic physical and behavioral conditions in PCPCHs will be necessary for the comparative effectiveness of the model's application. Therefore, it will be important to evaluate chronic disease burden levels in PCPCHs and the association between high and low chronic disease burden levels and outcomes. 
The development of the chronic disease burden measure for this research will be based on 10 markers of chronic conditions that persist and are different from acute conditions. The 10 markers of chronic conditions are cardiovascular disease, chronic heart failure, coronary heart disease, chronic kidney disease, asthma/COPD, diabetes, obesity, affective disorder, other behavioral health, and schizophrenia. The chronic disease burden measure will be developed consistent with prior health services literature on proxy health risk measures (Ash \& Ellis, 2012; Hornbook \& Goodman, 1996; Ash et al., 2000). Individual chronic disease burden will comprise the sum of the 10 markers of chronic conditions, while the average chronic disease burden of PCPCH practices will comprise the average of PCPCH practices' patient level chronic disease burden measures. The average chronic disease burden measure will be stratified into high and low average chronic disease burden levels and compared pre-post PCPCH recognition to their respective non-PCPCH comparators.

This relative effectiveness research will explore the following three questions:

1. What is the average chronic disease burden of PCPCHs, and how does their average chronic disease burden compare to the communities PCPCHs are in pre-post PCPCH recognition?

2. How do primary care expenditures change based on the chronic disease burden of PCPCHs?

3. Do PCPCHs that engage more high chronic disease burden patients have more reductions in ED and IP expenditures? 


\section{Theoretical Perspectives}

The theories guiding the development of this research are Risk selection in health care services delivery and considering the organization and delivery of health care as Complex Adaptive Systems (CAS). The theory of selection postulates that information asymmetry, heterogeneity of health risks, and the average-cost approach to reimbursement make it economically beneficial for agents to select 'good risks' based on the health conditions of patients (Arrow, 1963; Newhouse, 1996; Reinhardt, 1998). Evidence on the chronic disease burden levels in PCPCHs will provide insights on the engagement of chronic care patients in PCPCHs.

Health care delivery organizations including Medical Homes have been characterized as Complex Adaptive Systems. CAS organizations self-organize and adapt to changes based on internal models (rules) and signals from their environments (Crabtree, McDaniel, Miller, \& Stange, 1998; Litaker, Tomolo, Liberatore, Stange, \& Aron, 2006). Since most health care services are delivered in a 'market-based' environment, CAS suggests that PCPCHs will self-organize and adapt based on incentive systems in the delivery environment. The location of PCPCH providers, the level of health risks in their panels, and the primary care services offered will all be important to evaluating how primary care clinics that become PCPCHs are adapting to the policy changes. The results of this research will be analyzed from these theoretical perspectives. 


\section{Methodology}

This research is a quantitative outcome study and will be based on a natural experiment. Conceptually, the assignment to treatment and control groups in natural experiments arises from changes to policies, programs, or laws, rather than a formally randomized assignment to treatment and control groups. The effects of the exogenous changes to policies and laws on specific outcomes are measured by comparing groups that are affected (treatment) and unaffected (control) by such policy changes (Meyer, 1995). The effects of the implementation of PCPCHs in 2011 will be evaluated to determine the relative effectiveness of this policy change on the increases or decreases to Primary Care (PC) ED and IP expenditures. Economists use Differencein-Difference (D-in-D) methods to measure the exogenous variation from natural experiments. Other disciplines classify D-in-D methods as before-and after-designs with an untreated comparison group. D-in-D models will be developed to evaluate chronic disease burden levels in PCPCHs and their comparators pre-post $\mathrm{PCPCH}$ recognition; primary care expenditures by chronic disease burden levels in PCPCHs and their comparators pre-post PCPCH recognition; and ED and IP expenditures by chronic disease burden levels in PCPCHs and their comparators pre-post $\mathrm{PCPCH}$ recognition.

The outcome variables of this research will be evaluated between PCPCHs and their comparators: chronic disease burden levels (high versus low) of PCPCHs and their comparator groups; average primary care expenditures by chronic disease burden levels (high versus low) in PCPCH and their comparator groups; average ED 
and IP expenditures by chronic disease burden levels (high versus low) in PCPCHs and their comparator groups. The case-matching variables for $\mathrm{PCPCH}$ comparators will be age, gender, health insurance type, and zip code of patients. Each of these variables will be weighted, aggregated, and used to construct PCPCH comparators. The markers of chronic conditions in PCPCHs and their comparators will also be weighted, aggregated, and split into high and low average chronic disease burden groups for this research.

The intervention group will comprise recognized $\mathrm{PCPCHs}$, while the comparator groups will be constructed both from non-PCPCH primary care clinics in the state and from stratifying PCPCHs into high and low chronic disease burden PCPCHs. Primary care clinics that applied for PCPCH recognition but were not recognized as well as clinics currently applying for $\mathrm{PCPCH}$ recognition will be excluded from this study. The pre-policy period will comprise data on primary care patients in 2011, while the post-policy periods will comprise data from the first wave of PCPCH recognition (2012 to 2014). The following data sets will be used for this research: The All Payer All Claims (APAC) and PCPCH attestation data. Oregon's APAC data system provides complete and standardized patients' de-identified data on health care claims and eligibility from private, public, and third-party health plans and payers, including pharmacy claims and demographic information (Love, Custer, \& Miller, 2010). Data from the PCPCH program will include practice-level data on PCPCH attestation. 


\section{Chapter 2: Review of the Literature}

\section{History of the Medical Home Model}

Medical Homes (MH) by design are patient-centered, relationship-based health care delivery models that emphasize comprehensive primary care and the management of chronic conditions. The National Committee on Quality Assurance (NCQA) defines MHs as delivery models in which "patients establish ongoing relationships with personal physicians who lead teams at single locations and take collective responsibility for patient care, providing for the patients' health care needs and arranging for appropriate care with other qualified providers" (NCQA, 2011, p. 2).

Conceptually, the model emphasizes patient-centered approaches to empowering primary care provider teams to cultivate relationships with patients; use their knowledge and understanding of patients' health statuses, which have been developed over time to prevent diseases; detect diseases earlier; and coordinate and integrate care for patients. The evidence suggests that this approach to care reduces inappropriate (overuse and misuse) of ED and IP and improves the overall health of patients (Berwick, Nolan, \& Whittington, 2008; Berry \& Mirabito, 2009; Starfield \& Shi, 2004; Stange et al., 2010; Fisher, 2008; Glasgow et al., 2002). The model emerged around 1967 as part of the American Academy of Pediatrics care guidelines for patients who have special health care needs. During that time, MHs served as central locations for the storage and timely retrieval of patient information (Sia, Tonniges, Osterhus, \& Taba, 2004; Robert Graham Center, 2007). Due to the relative 
effectiveness of the model since introduction, a national initiative in 2004, "The Future for Family Medicine Project," which was supported by the American Academy of Physicians (AAP), the Institute of Medicine (IOM), and other primary care provider groups, identified the lack of emphasis on comprehensive primary care and began advocating for the broader application of the $\mathrm{MH}$ model. The renewed emphasis on MHs in 2004 was based on strengthening primary care delivery as part of broader health care delivery system reforms.

The MH model has been supported by various health policy proposals: Section 204 of the Tax Relief and Health Care Act of 2006 and the 2010 Patient Protection and Affordable Care Act (PPACA) provided guidelines for the model's implementation around the country to improve the quality of care, reduce overutilization of ED and IP, and reduce associated health care costs (PPACA, 2010; Collaborative, P.C.P.C., 2007). Variants of the model since 2004 have been introduced in different states, in different delivery systems, by different payers, and for different population groups. At the core of the model are the following attributes: patient-centered relationships, team-based approach to care, comprehensive primary care, chronic disease management, and payment reforms to incentivize care coordination and integration. The model continues to gain broad support around the country based on the relative effectiveness of its application. Some states have implemented variants of the model as part of their health care delivery system reforms, but there are differences in the model's implementation. Among the differences are semantics, accreditation and recognition, measurement, and 
population focus. Semantically, the model is referred to in some implementations as MHs, Health Homes, Primary Care Homes, or Patient-Centered Primary Care Homes. Recognition and accreditation of the model's implementation have been from states or national accreditation organizations such as National Committee on Quality Assurance (NCQA) and the Agency for Healthcare Research and Quality (AHRQ). Measurement thus far has been based on short-term measures policymakers focus on for policy decisions, such as ED and IP utilization and expenditures and health care costs. The population focus also includes pediatrics, including children who have special health care needs, adult patients, chronic care patients, and homeless patient populations.

Based on the focus of this research, this chapter will broadly review literature on two core tenets of the model: primary care and chronic disease management and the different applications of the model and their respective outcomes. The following themes and headings will guide the literature review: the history and landscape of the model's application; the variations of the model; Oregon's Patient-Centered Primary Care Home (PCPCH) model; the main components of Oregon's model and how PCPCHs relate to other MH models; evidence of improvements in PC, ED, IP, and health care costs; outcomes from the application of the model; the importance of health risks to the model's application and why this study focuses on evaluating health risks in PCPCHs; the theoretical lens for thinking about health risks and outcomes in PCPCHs; and how the theories help explain the model's application and 
potential research results. This chapter will conclude by restating the research questions and hypotheses.

\section{The Patient-Centered Medical Home (PCMH) Model}

The Patient-Centered Medical Home (PCMH) model is often referred to as $\mathrm{MH}$ in the literature. Historically, the endorsement of the Joint Principles of the PCMH model in 2007 by AAP, IOM, and other physician groups was important to the model's reintroduction because it helped establish a consensus of standards for the model's implementation across the country. The Joint Principles established the following framework for the model's application:

- Patients having ongoing relationships with personal physicians

- Physician directed medical practice

- Whole person orientation of care

- Care coordination and integration

- Emphasis on quality and safety

- Enhanced access for patients

- Payment reforms to recognize value added patient care

The evidence suggests that in addition to the semantic differences in how the model is described, there are other differences in how the model has been implemented, including performance measurement and recognition/certification of the model. The following are specific, but not exhaustive examples of implementations of the model in different delivery systems and settings: 
Closed organizational systems: The Veteran's Administration - Patient Aligned Care Teams (PACTs) (Hebert, P. L., Liu, C. F., Wong, E. S., Hernandez, S. E., Batten, A., Lo, S., ... Fihn, S. D. (2014); Nelson, K. M., Helfrich, C., Sun, H., Hebert, P. L., Liu, C.-F., Dolan, E., ... Hernandez, S. E. (2014); Yoon, J., Liu. C.F., Lo, J., Schectman, G., Stark, R., Rubenstein, L.V., \& Yano, E.M (2015).

Private Health Maintenance Organizations (HMOs): Geisinger Health System PCMH (ProvenHealth Navigator) (Maeng, D.D., Khan, N., Tomcavage, J., Graf, T.R., Davis, D.E., \& Steele, G.D (2015).

Vulnerable and underserved populations: Sugerman, J.R., Phillips, K.E., Wagner, E.H., Coleman, K., \& Abrams, M.K (2014).

Chronic care patients: Neal, J., Chawla, R., Colombo, C.M., Snyder, R.L., \& Nigam, S (2015).

All classes of patients in different states: in Oregon (Rissi, J., Gelmon, S., Saulino, E., Merrithew, N., Baker, R., \& Hatcher, P (2015); Gelmon, S., Wallace, N., Sandberg, B., Petchel, S., \& Bouranis, N (2016); in Vermont (Jones, C., Finison, K., McGraves-Lloyd, K., Tremblay, T., Mohlman, K.M., Tanzman, B., Hazard, M., Maier, S., \& Samuelson, J (2015)); in multi-states (Nielsen, M., Buelt, L., Patel, K., \& Nichols, L. M (2016).

The model has also been implemented using various traditional and alternative value-based payment incentives. Among the examples of such implementations include:

Single payers: VA's PACTs (Randall, 1., Mohr, D.C., \& Maynard, C (2014)). 
Fee-For-Service (FFS) only payments: (Pines, J.M., Keyes, V., Van Hasselt, M., \& McCall, N. (2015)).

Mixed payments: (FFS, per member per month services (PMPM), shared savings and other forms of incentive arrangements) (Rosenthal, M.B., Alidina, S., Friedberg, M.W., Singer, S.J., Eastman, D., Li, Z., \& Schneider, E.C. (2016)).

Measurement and performance: Most $\mathrm{MH}$ implementations have focused on measuring utilization and expenditures based on the main measures that policymakers focus on when they evaluate the relative effectiveness of the model's application, such as ED, IP, PC, costs (per member per month services [PMPM], total costs of care, and expenditures) Gelmon, S., Wallace, N., Sandberg, B., Petchel, S., \& Bouranis, N (2016). Other evaluations focus on the level of "medical homeness" Lemak, C.H., Nahra, T.A., Cohen., G.R., Erb N.D., Paustian, M.L., Share, D., \& Hirth, R.A (2015).

Certification/accreditation. There are currently four main national and various state-level accreditation bodies. The national organizations are the Accreditation Association for Ambulatory Health Care, the Joint Commission, the National Committee on Quality Assurance (NCQA), and the Utilization Review and Accreditation Commission. Oregon, Colorado, Massachusetts, Pennsylvania, Vermont, and Minnesota are among states that have developed state-level recognition/accreditation standards.

In addition to emphasizing PCMH demonstrations around the country, the Affordable Care Act also supported Health Home (HHs) demonstrations. HHs are 
variants of MHs and are defined as population-based health care delivery models for coordinating and integrating physical and behavioral care for chronic condition patients in Medicaid (PPACA, 2010). According to section 1945(h) of the Act, HH populations must comprise patients who have the following health risks: "at least two chronic conditions; one chronic condition and at risk for a second health condition; patients who have "serious and persistent mental health conditions"' (p. 8). Though MHs and HHs are similar in their implementation goals and intended outcomes, the models are different in several areas that need to be delineated for clarity.

Conceptually, the two models (MHs and HHs) are similar in their organization around the Joint Principles, emphasis on comprehensive primary care and prevention, care coordination and integration, chronic disease management, interdisciplinary team care, relationship-centered care, and payment reforms to incentivize comprehensive chronic care. Their main differences, highlighted in Table 2.1, are population focus, provider requirements, and emphasis on care coordination and integration across physical and behavioral health conditions. Health Homes tend to focus on Medicaid patients who have various levels of chronic conditions. The emphasis on care coordination and integration across physical and behavioral health conditions is intended to improve the quality of patient care and outcomes. Health Homes are also recognized by state Medicaid agencies. In contrast, PCMHs are recognized by both state agencies and national accreditation agencies. Both models use payment incentives, but the incentives are structured differently to reflect different patient needs and primary care improvement priorities for states using these models. 


\section{Table 2.1}

Primary Differences between Patient-Centered Medical Homes and Health Homes

\begin{tabular}{|c|c|c|}
\hline & Patient-Centered Medical Homes & Health Homes \\
\hline Patient Population(s) & $\begin{array}{l}\text { Provides care to all patient } \\
\text { populations across their life spans. }\end{array}$ & $\begin{array}{l}\text { Provides care to individuals with various levels of } \\
\text { approved chronic conditions. }\end{array}$ \\
\hline Main Providers & $\begin{array}{l}\text { Typically defined as physician-led } \\
\text { primary care practices, but also mid- } \\
\text { level providers such as nurse } \\
\text { practioners. }\end{array}$ & $\begin{array}{l}\text { May include primary care practices, community } \\
\text { mental health centers and organizations, } \\
\text { addiction treatment centers and providers, } \\
\text { Federally Qualified Health Centers, Health Home } \\
\text { agencies, and other chronic care providers. }\end{array}$ \\
\hline Payer Types & $\begin{array}{l}\text { All payers, including Medicaid, } \\
\text { commercial insurance, and private } \\
\text { payers. }\end{array}$ & Currently a Medicaid-only construct. \\
\hline $\begin{array}{l}\text { Organization of Care } \\
\text { Delivery }\end{array}$ & $\begin{array}{l}\text { Team-based and whole person } \\
\text { orientation with a focus on } \\
\text { coordinating primary care services, } \\
\text { including referral and tracking, } \\
\text { treatment guideline adherence, } \\
\text { electronic prescription, and provider- } \\
\text { patient communication. }\end{array}$ & $\begin{array}{l}\text { Team-based and whole person orientation with a } \\
\text { strong focus on coordinating and integrating } \\
\text { behavioral health care that includes substance } \\
\text { abuse treatment, social and family support, and } \\
\text { other services such as nutrition, home health care, } \\
\text { and transitional care. }\end{array}$ \\
\hline Technology Use & $\begin{array}{l}\text { Health Information Technology for } \\
\text { coordinating and integrating care } \\
\text { across the continuum of care, } \\
\text { including referrals. }\end{array}$ & $\begin{array}{l}\text { Health Information Technology for coordinating } \\
\text { and integrating care and services across the } \\
\text { continuum of care, including in-home care such } \\
\text { as remote monitoring in patient homes. }\end{array}$ \\
\hline Provider Requirements & $\begin{array}{l}\text { State Medicaid and National } \\
\text { Committee on Quality Assurance } \\
\text { determined. }\end{array}$ & State Medicaid agency determined. \\
\hline Incentive Systems & $\begin{array}{l}\text { Alternative and bundled payments } \\
\text { usually based on Per Member Per } \\
\text { Month comprehensive care designed } \\
\text { to improve the value of care. }\end{array}$ & $\begin{array}{l}\text { Per Member Per Month payments for required } \\
\text { services, as well as for care coordination and } \\
\text { integration. }\end{array}$ \\
\hline
\end{tabular}

Source: Smith (2011).

While various states have implemented variants of HHs or PCMHs or both, it can be argued based on its design and implementation that Oregon's Patient-Centered 
Primary Care Homes (PCPCH) is a hybrid model that attempts to integrate both HHs and MHs to provide care for the state's entire population and includes all provider and payer groups in the state. The next section of this chapter will review Oregon's PCPCHs and the differences between PCPCHs and other state level models.

\section{Oregon's Patient-Centered Primary Care Home (PCPCH) Model}

PCPCHs emerged from a legislative initiative for broader health system

transformation in the state (HB 2009). The state's health care agency, the Oregon

Health Authority (OHA), began recognizing PCPCHs in 2011. It was projected that at least 75 percent of the state's population would receive their health care services in PCPCHs by 2015 (OHA, 2010). Since November 2011 over 600 primary care providers have been recognized as PCPCHs with more providers in line for recognition. Over 100 of the state's PCPCHs have also been cross-recognized by the NCQA (NCQA, 2014). Oregon's PCPCH recognition standards were developed through a state-level consensus process, but the standards are based on the Joint Principles framework and the NCQA national standards. Among Oregon's core recognition standards are comprehensive primary care, care coordination and integration for chronic care patients, quality improvement, and the delivery of patientand family-centered care. The development and use of state level recognition standards helped emphasize state-level priorities for transforming primary care delivery. Table 2.2 lists the main components of NCQA and Oregon's PCPCH recognition standards. 
Table 2.2

Primary Standards for Patient-Centered Medical Home Certification

\begin{tabular}{l|l}
\hline \multicolumn{1}{c}{ NCQA } & \multicolumn{1}{c}{ PCPCH } \\
\hline Enhance Access and Continuity of Care & Access to Care \\
Identify and Manage Patient Populations & Accountability for Patient Care \\
Plan and Manage Care & Comprehensive Care \\
Provide Self-Care and Community Support & Continuity of Care \\
Track and Coordinate Care & Care Coordinate and Integration \\
Measure and Improve Performance & Patient and Family-Centered Care \\
\hline
\end{tabular}

Source: (OHA, 2013; NCQA, 2011)

It can be inferred from Table 2.2 that the standards are similar. Both PCPCHand NCQA-recognized Medical Homes are tiered based on attestation to standards met and performance improvements after initial attestation. The tier system is based on numerical weights attached to each attestation standard. Clinics that apply for recognition must attain a minimum score to be recognized at tier 1; higher than minimum scores qualify clinics into higher tiers. For example, in addition to 10 “must-pass" standards for all PCPCH tiers, tier 1 clinics must achieve an aggregate between 30 and 60 points, tier 2 between 65 and 125, while tier 3 requires at least 130 points. Oregon has recently introduced additional tiers for high performing clinics such as tier 3-star, tier 4 and tier 5 or higher (OHA, 2013). 


\section{PCPCH and other Medical Home Models.}

While all states and delivery systems have implemented variants of MHs and HHs (Takach, Gauthier, Sims-Kastelein, \& Kaye, 2010), it can be inferred from the evidence that most models are, by their design and intended outcomes, more similar than different. Stange et al. (2010) corroborates this inference with their argument that all MH models contain four main elements: comprehensive primary care, new ways for organizing health care delivery, strengthening practices internal capacities to provide effective care, and different incentives for health care delivery. A comparison between Oregon and NCQA (most-used recognition standards) from Table 2.2 confirms Stange et al.'s (2010) argument that the models are more similar than different. Among the broad differences are the emphasis on model components, the focus on different population groups, the structure of enhanced provider payments, the choice of provider recognition through standards developed at the state or national levels (or both), and the weighting of the recognition standards.

Even though there are different state-level recognition standards for MHs and HHs, most recognition agencies emphasize and allocate the most points to the following standards: care integration and coordination for patients with various levels of chronic physical and behavioral health conditions, Health Information Technology (HIT) use for care coordination and care management purposes, patient engagement, and quality improvement. The differences are revealed in the differential point allocation to different model elements, the choice for state-developed or national agency recognition standards, and their intended outcomes. Oregon and NCQA 
recognition classify clinics into tiers but use different weighting. For example, while the weights for tier 1 clinics are similar, the total weights for qualifying into tier 2 and tier 3 for PCPCH clinics are between 65 and 125, and 130 and up, respectively. However, NCQA's requirements for tier 2 and tier 3 are between 60 and 80, and 85 and 100, respectively (OHA, 2010; NCQA, 2012). The weights for care coordination, integration, and patient engagement are similar for both models. To recognize adaptations of the model and to appropriately align incentives, Oregon has recently increased the tier ratings for PCPCH clinics to recognize higher performing PCPCHs. PCPCH clinics can now receive tier 4 and tier 5-star ratings in Oregon.

\section{PCPCH and other States' Medical Home Programs.}

At the core of Oregon's PCPCH model is the goal of improving the delivery of comprehensive and patient-centered primary care services and the management of various levels of chronic conditions upstream, especially for Medicaid patients. Different levels of enhanced payments are used to encourage teams of providers across the care continuum to comprehensively coordinate and integrate care for patients, especially those who have various levels of chronic physical and behavioral health conditions. While most Medical Homes are similar in design, there are inherent differences in their applications and outcomes. Comparing Oregon's PCPCH model to similarly designed state-level Patient-Centered Medical Home (PCMH) models, namely Colorado, North Carolina, Minnesota, Vermont, Oklahoma, Massachusetts, and Pennsylvania reveal differences such as the varying emphases on the model's 
application, patient focus, and use of payment incentives for care coordination and integration.

Oregon vs. Colorado. Colorado's program, begun in 2009, differs from Oregon's PCPCH in terms of population focus and payer types. Colorado has a single-payer program for the state's high health-risk children enrolled in Medicaid or the children's health insurance program (CHIP) (Colorado Department of Health Care Policy, 2012). Colorado's program also uses both state and NCQA recognition standards. Oregon's PCPCH, on the other hand, focuses on providing care to all patient population groups in the state, with a specific emphasis on Medicaid populations, but the model is applied across all payer groups. Unlike Oregon's multiple tier levels, MHs in Colorado are recognized under a single tier (Takach, 2011).

Oregon vs. North Carolina. Community Care of North Carolina (CCNC), one of the oldest programs in the country, is North Carolina's MH variant. The program began in 1998 and was incrementally developed into a state-level MH for managing chronic conditions. It is estimated that 80 percent of Medicaid patients, comprising about 10 percent of the state's population who are considered high risk because of their chronic conditions, receive care from CCNC (Steiner et al., 2008). The recognition of practices in CCNC is based on NCQA standards (Trapp, 2010). CCNC is like Oregon's PCPCH in its program design and recognition standards but different in its approach to recognizing clinics and patient focus. Unlike Oregon, CCNC uses NCQA recognition standards and focuses primarily on chronic care patients. 
Oregon vs. Minnesota. Minnesota's Health Care Home program started in 2010. Health Care Homes are like PCPCHs in their design, recognition, and population focus. Both programs use multi-payers and state-developed recognition standards, and both focus on all patients. Minnesota's program, however, is different because all providers qualify under a single tier, while patients are stratified into "complexity tiers" based on their number of chronic conditions. There are currently five distinct complexity tiers. As part of this patient stratification process for payments, clinics that coordinate and integrate physical and behavioral health care for high-risk patients who have multiple chronic conditions receive additional enhanced payments (Minnesota Department of Health [MDH] 2012). Minnesota's model currently pays the highest enhanced payments for care coordination, between $\$ 10.14$ and \$79.05 (Takach, 2011). On the other hand, comprehensive payments for PCPCHs vary by payers. It ranges between $\$ 10$ and $\$ 25$ for the Center for Medicare and Medicaid (CMS) payments, which are discriminated upon based on practice tier levels (PCPCH, 2013). These amounts are based on available information; because payment rates are adjusted periodically, the amounts may change over time.

Oregon vs. Vermont. Vermont's Blueprint for Health (BPH) was launched in 2006 as part of the CMS multi-payer Advanced Primary Care Practice demonstration. $\mathrm{BPH}$ is similar in design and scope to $\mathrm{PCPCH}$. For example, both programs provide care to all populations, use multi-payers, and provide enhanced provider payments for care coordination and integration. BPH is different in the use of NCQA recognition and multidisciplinary community health teams to provide care for a panel of 20,000 
patients in each of its three rural demonstration settings (Bielaszka-DuVernay, C. 2011).

Oregon vs. Oklahoma. Oklahoma's Patient Centered Medical Home (PCMH) SoonerCare Choice delivery program began in 2009 and focused on improving the delivery of comprehensive primary care as a strategy for containing health care costs in the state. Like Oregon's PCPCH program, Oklahoma classifies PCMH providers into three tier levels but with different classifications: entry, advanced and optimal. SoonerCare choice and PCPCH are similar in other areas as well. Both programs use value-based payment incentives to incentivize care coordination and integration. Payment incentives are differentiated based on provider tier levels, both programs are based on state developed recognition standards, and focus on all patients, especially, beneficiaries of Medicaid (Takach, 2011).

Oregon vs. Massachusetts. The Patient-Centered Medical Home Initiative (PCMHI) in Massachusetts began in 2009 with 46 primary care providers including community health centers. The PCMHI program was designed to reduce fragmentation in care delivery and health care costs by focusing on the following: behavioral health integration into primary care and chronic disease management. Massachusetts PCMHI is like Oregon's PCPCH program in terms of their use of a mixture of payment incentives to coordinate and integrate care for patients. But the PCMHI program is different in terms of accreditation - a mix of state developed and NCQA standards. PCMHI providers are also not differentiated based on practice tier levels (Cabral, Sefton, \& Anderson, 2013). 
Oregon vs. Pennsylvania. The Pennsylvania Chronic Care Initiative (PCCI), a state-level MH variant, began in 2009 as a collaborative initiative by the state's largest commercial health plans and Medicaid-managed care plans for 32 main medical practices in the state. Practices were recognized as Patient-Centered Medical Homes (PCMH) based on NCQA standards, with financial support from the state for practice recognition. The PCCI initiative was focused on improving the care for chronic care patients. PCCI is like Oregon's PCPCH program in their use of valuebased payment incentives for coordinating and integrating care and their state-level implementation and support. But PCCI is different in its focus on chronic care patients and use of NCQA accreditation standards.

Summary. It can be inferred broadly from the sampling of $\mathrm{MH}$ applications in different states that the applications of the model are similar but different in other areas. All models emphasize primary care, use of the chronic care model for comprehensive care coordination and integration, and alignment of payment incentives to ensure that providers focus on health risks that drive utilization and expenditures. Proponents of the model argued that emphasizing the above elements in health care delivery may improve care and outcomes by engaging patients who have chronic conditions and whose utilization disproportionately drive up expenditures (Fisher, 2008; Abrams, Davis, \& Haran, 2009; Berwick \& Hackbarth, 2012).

While preliminary evidence from variants of the model's application in different delivery systems and states suggests improvements in some process and outcomes metrics of care (specific examples are provided below), the levels of health 
risks in MHs have not been studied. The results of this research will provide important contributions to this field in terms of levels of high chronic disease burden patients enrolled in PCPCHs and other MHs. Evaluating chronic disease burden levels in PCPCHs and the association between high chronic disease burden PCPCHs and ED and IP expenditures will provide insights into the relative effectiveness of the application of the model.

\section{Evidence of Improvements in Cost-Sensitive Outcomes of Care}

Bending the trajectory of health care expenditure growth is one of the Triple Aim and a goal of PCPCH implementation and health care reforms in general. MHs are important in achieving health reform goals because of the expectations that providing comprehensive primary care and disease management upstream in ambulatory settings will reduce the pressure on overuse and misuse of ED and IP utilization. Most evaluations of the model have thus far focused on measuring short term improvements in ED and IP use (Rittenhouse, Thom \& Schmittdiel, 2010). While the focus on short-term improvements are necessitated in part by the short-term history of the model's application and the availability of data, it can be argued that the model's long-term sustainability will depend on demonstrable short-term improvements. This research will therefore focus on the chronic disease burden of PCPCHs and short-term improvements in ED and IP use.

The next section will review cost-sensitive outcomes from the main elements of the model, namely primary care and the chronic care model. While there are other elements of the model that are associated to improved outcomes, this review focuses 
on primary care and the chronic care model because they are foundational to all applications of the model across delivery systems. This section will also review evidence of the application of the model for different population groups: pediatric and adult populations, those in Integrated Delivery Systems (IDS), and those in the following state-level programs: North Carolina, Vermont, Colorado, Minnesota, Oklahoma, Massachusetts, and Pennsylvania. While variants of Medical Home initiatives are operational around the country (Hing, Kurtzman, Lau, Taplin, \& Bindman, 2017), the afore mentioned state-level Medical Home programs were selected based on the following criteria: the early adoption and implementation of the model in those states; the use of state-developed recognition standards, or a mixture of state-developed and NCQA recognition standards; and the use of multi payer provider payment incentives. The evidence from these state-level programs is therefore not representative of all state programs or $\mathrm{MH}$ implementations.

\section{The Cost-Sensitive Benefits of Primary Care.}

Providing comprehensive primary care for patients with various levels of chronic physical and behavioral health conditions adds value because the elements of primary care, such as accessibility, care coordination, integration, and appropriate referrals, have been found to reduce the overuse and misuse of ED and IP utilization. Shi and Singh (2008) posited that "an ideal system of health care delivery ought to have a strong primary care base, and for the system to be effective, primary care should be closely integrated with adequate and timely specialized services" (p. 249). 
Accordingly, most MH models, including PCPCHs, emphasize comprehensive primary care.

Empirically, Basu, Friedman and Burstin (2004) found that patients in private Health Maintenance Organizations (HMOs) who received well-coordinated primary care services had consistently higher reductions in preventable hospitalizations than patients who received uncoordinated primary care services in private Fee-For-Service (FFS) only programs. In their study on Medicaid patients in Colorado who received care from Federally Qualified Health Centers (FQHCs) and from private FFS providers, Rothkopf, Brookler, Wadhwa, \& Sajovetz (2011) found that primary care in FQHCs resulted in less IP and ED utilization than in FFS systems.

In Oregon, a study on the expansion of primary care through the Oregon Health Plan (OHP) found that "preventable hospitalization rates varied inversely with access to primary care but correspondingly with access to hospital care" (Saha, Solotaroff, Oster, \& Bindman, 2007, p. 717). This evidence suggests that increasing access to primary care reduced the incidence of preventable hospitalizations. The evidence further supports the association between the receipt of primary care and improved ED and IP utilization. Maeng et al. (2012) found that Geisinger Health System's dual approach to expanding primary care access and integrating primary care services across various levels of care for chronic care patients improved utilization and patient outcomes: "[M]oving resources further "upstream" to primary care settings reduced "downstream" costs from highest acuity settings" (p.150). Several other studies corroborate the association between comprehensive primary 
care and reduced ED and IP utilization (Epstein, 2001; Falik et al., 2005; Franks, Cameron, \& Bertakis, 2003).

Improving the Value of Primary Care. Despite the association between the receipt of primary care and improved outcomes, primary care has historically been less emphasized in health care delivery. This primary care 'chasm' has arguably contributed to the overuse, misuse, and underuse of health care services, along with rising health expenditures (Safran, Tarlov, \& Rogers, 1994; The Commonwealth Fund, 2012; Berry \& Mirabito, 2009; Davis, Schoen, \& Stremikis, 2010). Among the arguments for under-emphasizing primary care included the supply of the primary care workforce and the cost-effectiveness of primary care and prevention.

Opponents of MHs have cited these and other arguments as reasons for not supporting an expanded application of the model. They argue that shortages in the primary care workforce will exacerbate workforce burnout and contribute to worsening outcomes (Berenson \& Rich, 2010; Bernstein, Chollet, Peikes, \& Peterson, 2010). But the evidence from the model's application suggests that the use of primary care teams working collaboratively with primary care physicians (PCPs) in MHs can improve patient care and outcomes (Sylvia, 2008; Bodenheimer, Chen, \& Bennett, 2009). Other evidence suggests that while the supply of PCPs can be improved, arguments about PCP shortages are overstated because current primary care workforce levels are adequate to sustain health care production in MHs: "[I]nvesting in a major expansion of the physician workforce is a distraction from what has already been shown to be effective" (Goodman \& Grumbach, 2008, p. 337). 
While improving the supply of PCPs will be useful, it can be inferred that the use of primary care teams would sustain the model's workforce needs. Regarding the cost-effectiveness of primary care and prevention, a core argument by opponents is the cost-effectiveness of providing preventive care to people who may never be afflicted by the conditions for which they receive preventive care. In their study on hypertension care, Weinstein \& Stason (1976) suggested that the aggregate costs of prevention services were often greater than their accrued benefits in Quality Adjusted Life Years (QALY). Russell (1986) and Cohen, Neumann, \& Weinstein (2008) also suggested that some prevention services were associated with increased medical spending.

Providing effective primary care in PCPCHs, however, can be cost-effective and cost-saving. Goetzel (2009), Russell (2009), and Enthoven, Crosson, \& Shortell (2007) all emphasized the important benefits of primary care and prevention. Goetzel (2009) argued that opponents of prevention fail to separate the different types of preventive care (primary, secondary and tertiary) and their respective benefits. $\mathrm{He}$ posited that "pitted against the cost of medical treatment, prevention offers a good return on investment" (p. 38). Russell (2009) observed that "prevention can be cost effective and sometimes cost saving components of managing established chronic conditions" (p. 44). Enthoven, Crosson, \& Shortell (2007) emphasized the "interdependencies" in health care delivery to support their argument that care coordination and integration for chronic care patients in primary care settings 
improves health care value by reducing patient demand and use of relatively expensive ED and IP utilization.

It can be inferred from the above evidence on primary care that comprehensive primary care can add value because primary prevention can proactively reduce the incidence of some chronic conditions, while secondary and tertiary care can help manage diseases more cost-effectively. An environmental scan of health care needs underscores this assertion. It is estimated that 157 million Americans will be living with at least one chronic condition by 2020 . The number of people who will be living with multiple chronic physical and behavioral health conditions is also expected to grow from 63 million in 2005 to about 81 million by 2020 (Wu \& Green, 2000).

This growing incidence and prevalence of chronic conditions supports the potential benefits and value of integrating comprehensive primary into MHs. Emphasizing comprehensive primary care in MHs can help engage patients whose health risks predispose them to higher health care utilization and expenditures. The growing burden of chronic conditions also suggests the importance of a comprehensive and integrated approach to managing chronic conditions in MHs. The next section will focus on the management of chronic conditions and the use of the chronic care model for chronic disease management.

\section{The Cost-Sensitive Outcomes from Chronic Disease Management}

Historically, several chronic disease management programs have been applied to managing chronic diseases, namely the report card initiative, chronic disease 
management by private companies (CDMC), and the Chronic Care Model (CCM). The report card initiative became ineffective shortly after introduction (Marshall, Shekelle, Leatherman, \& Brook, 2000). CDMCs provided care for single chronic conditions in patients' homes through nurse visits, health education, and the use of various health information technologies (HIT) to support this care process (Cassalino, 2005). The CCM, on the other hand, is considered a generic model that integrates evidence-based elements of primary care, self-management education, HIT, and team care for managing chronic conditions (Coleman, Austin, Brach, \& Wagner, 2009).

Chronic Disease Management Companies (CDMC). The evidence for the comparative effectiveness of CDMCs is mixed. While programs have demonstrated improvements in some patient outcomes, most of the contributions to reduced ED and IP use have not been statistically significant (Ouwens, Wollersheim, Hermens, Hulscher, \& Grol, 2005). A comprehensive review of CMS FFS programs since 1999 revealed that while CDMC programs improved patient behavior, quality of care, and patient satisfaction, only 20 percent of their programs achieved reductions in IP use or projected cost targets (Bott, Kapp, Johnson, \& Magno, 2009). Other evaluations arrived at similar conclusions. For example, studies on congestive heart failure (CHF), chronic obstructive pulmonary disease (COPD), asthma, diabetes, and depression all corroborated the improvements in quality of care but suggested that the improvements did not translate into significant ED and IP use and health care costs (Vogeli et al., 2007; Mattke, Seid, \& Ma, 2007; Coleman, Austin, Brach, \& Wagner, 2009). Based on their meta-analysis of CDMC initiatives, Mattke, Seid, \& Ma (2007) 
concluded that "support for population-based disease management is more an article of faith than a reasoned conclusion grounded in well researched facts" (p. 675). This conclusion was corroborated by a Congressional Budget Office analysis on CDMCs (CBO, 2004).

The Chronic Care Model (CCM). The CCM has been used in health care delivery since the 1980s. The model is based on a process of care improvements for chronic care patients at the practice level. The elements of CCM that are associated to improved outcomes of care include multidisciplinary teams (Coleman, Austin, Brach, \& Wagner, 2009); self-management support (Bodenheimer, MacGregor, \& Sharifi, 2005); and HIT (Sperl-Hillen et al., 2004). The evidence suggests that implementing elements of the CCM improved care processes and some outcomes of care, for example, lipid-lowering and angiotensin-converting enzymes for CHF patients, and ED and IP utilization (Asch et al., 2005; Sochalski et al., 2009; Pearson et al., 2005; Fireman, Bartlett, \& Selby, 2004; Russell, Orleans, Wagner, Curry, \& Solberg, 2001); and QALY for diabetes and asthma care in several delivery settings (Huang et al., 2007; Eddy, Schlessinger, \& Kahn, 2005; Brown, Peikes, \& Chen, 2007; Gunterman, 2007). Among other improvements from implementing the CCM included lowering the risk of cardiovascular disease in diabetes patients (Glasgow et al., 2002; Vargas et al., 2007).

Other studies, however, question the model's association to improved ED and IP use and cost outcomes. A meta-analysis by Grumbach \& Bodenheimer (2004) and Orszag \& Ellis (2007) suggested that implementing CCMs resulted in increased 
utilization and expenditures. Opponents of the model's feasibility argue that improvements in processes and outcomes of care are not statistically significant in controlled evaluations. For example, a randomized controlled trial of a CCM in pediatric asthma care suggested that there were no significant differences between groups regarding the outcomes of asthma care (Homer et al., 2005). A diabetes study also found short- to intermediate-term improvements in process outcomes after two years but no improvements in costs (Chin et al., 2007). Hroscikoski et al. (2006) and Solberg et al. (2006) also found CCM implementation challenges.

Summary of evidence. The evidence thus far suggests that while there are variants of the $\mathrm{MH}$ models, the models are more similar than different. The similarities and differences are evident from Table 2.1, which delineates the differences between MHs and Health Homes, and from Table 2.2, which compares NCQA and PCPCH recognition standards. Stange et al.'s (2010) conclusion captures the model's application thus far, specifically that all MH models contain four main elements: comprehensive primary care, new ways for organizing health care delivery, stronger internal capacities for practices to provide effective care, and different incentives for health care delivery.

A review of the evidence of the core components of the model-primary care and chronic disease management—also suggests that primary care is associated with improvements in outcomes of care. The evidence also suggests the value of comprehensive primary care to the model's application. The evidence on chronic disease management, however, is mixed. Fragmentation in delivery, the focus on 
single disease conditions, and the lack of well-aligned payment incentives for chronic disease management are among the problems identified (Grumbach \& Bodenheimer, 2004; Orszag \& Ellis, 2007). It can be argued from an economic perspective that aligning payment incentives to support chronic disease management will be necessary for engaging chronic care patients and for improving outcomes.

Oregon's PCPCHs are designed to provide care to all patient populations, and according to a Health Management Associates (HMA) report on health care transformation in Oregon, the implementation of PCPCHs statewide can reduce health expenditures by about $\$ 4.5$ billion in 10 years (HMA, 2012). Since chronic care patients are significant drivers of health care expenditures, it can be argued that their enrollment in PCPCHs can help achieve the projected savings. While the design and implementation of PCPCHs focuses on all patients, this research proposes that the model's capacity to reduce the overuse and misuse of ED and IP and associated expenditures will be enhanced by engaging more high chronic disease burden patients in PCPCHs.

Variants of the MH model have so far been implemented broadly for different population groups, including pediatric and adult populations, in different Integrated Delivery Systems (IDS), and in various states. Reviewing outcomes from the different implementations of the model will help determine the relative effectiveness of the model's application and whether the model's implementation is focused on high chronic disease burden patients and associated to reductions in ED and IP utilization and expenditures. The next sections of this chapter will review evidence on 
outcomes from the different applications of the model in each of the above-mentioned settings.

\section{Improved Cost-Sensitive Outcomes from Pediatric Populations}

From the first application of the model for Children with Special Health Care Needs $(\mathrm{CSHCN})$ to the current application for all pediatric patients, the evidence shows some reductions in ED and IP use for pediatric patients. The improved outcomes were higher among pediatric patients who had chronic conditions, and when clinics had higher Medical Homeness (MH) index scores. Higher index scores suggested that the clinics integrated the core components of the model and met quality improvement benchmarks. Cooley, McAllister, Sherrieb \& Kuhlthau (2009) tested the hypothesis that the "application of the model in primary care practices is associated to decreased utilization and increased patient satisfaction" (p. 358). The intervention group was pediatric patients who had chronic conditions; the results suggested that clinics with higher $\mathrm{MH}$ index scores reduced IP and ED use and improved patient satisfaction. Diedhiou, Probst, Hardin, Martin \& Xirasager (2010) also found that asthma care in MHs for Children with Special Health Care Needs (CSHCN) resulted in reduced ED use by 1.2 percent for a population of 14,916 patients.

Klitzner, Rabbitt, \& Chang (2010) found evidence to support the specific components of the model that drove improvements in ED and IP use. Their study on caring for complex pediatric patients showed that effective care coordination resulted in a 1.1 percent reduction in ED use but no significant improvement in IP use for the 
same population. McGrath, Laflamme, Schwartz, Stransky \& Moeschler (2009) also found in a national survey on $\mathrm{CSHCN}$ that having an $\mathrm{MH}$ was the strongest predictor of whether children had genetic counseling services for autism spectrum disorder, down syndrome, and other behavioral health conditions. Integrating effective prevention and health education for chronic care patients in the model also resulted in improved ED and IP use (McGrath et al., 2009). It can be inferred from the evidence that the application of the model in pediatric settings improved some outcomes. The improvements in outcomes were significant when clinics were well organized in terms of higher $\mathrm{MH}$ index scores and focused on care coordination and integration.

\section{Improved Cost-Sensitive Outcomes from Adult Populations}

While the evidence for improved outcomes from implementing the model for adult populations is mixed, the evidence nonetheless corroborates the benefits of the model's application for high chronic disease burden patients. Ferrante, Balasubramanian, Hudson, \& Crabtree (2010) found that adult patients who had chronic conditions and were enrolled in MHs received well-coordinated primary care services and had less ED and IP use. Hearld \& Alexander (2012) found that "a one standard deviation increases in the level of patient-centeredness of care received was associated with a 4.6 percent decrease in ED utilization" (p. 568) for adult patients who had chronic conditions. Savage, Lauby \& Burkard (2013) and Ferrante, Cohen \& Crosson (2010) evaluated the use of patient navigators for care coordination;

Alexander, Hearld, Mittler, \& Harvey (2012) studied the use of patient-centered approaches to managing chronic conditions. These studies corroborate the importance 
of care coordination and integration to both the model's application and reduced ED and IP expenditures. Flottemesch et al. (2012) and Fontaine, Flottemesch, Asche \& Solberg (2011) also found in 27 certified practices in Minnesota that utilization, total costs of care, and inpatient costs for type-2 diabetes were significantly lower. Patients in recognized $\mathrm{MH}$ practices were also less likely to use ED or IP care services. In their study on comprehensive ED use by chronic care patients in MHs, Guy et al. (2013) found that "the largest reductions in ED visits were concentrated among chronic care patients with diabetes and hypertension" (p. 24-26).

While implementing the model for adult patients in some cases improved ED and IP use, the health-risk levels of patients who received care determined the outcomes. Dorr, Wilcox, Brunker, Burdon, \& Donnelly (2008) found that while care management plus, an HIT decision support, improved care for patients with severe chronic conditions and reduced mortality in the intervention group, the initiative did not significantly reduce ED or IP utilization or health care costs for patients who had severe chronic conditions. Jackson et al. (2013), Weaver et al. (2013), and Freidberg et al. (2014) all found that while patient-level outcomes of care improved for chronic care patients enrolled in MHs, the improvements did not translate into reductions in ED and IP use for patients who had chronic health conditions.

Based on their evidence, Freidberg et al. (2014) argued that "HM interventions may need further refinement" (p. 815). The varying effects of health risks on outcomes suggests the need for further evaluation of the applications of the model. Insights into the distributions of health risks and the differential effects of 
health risks on outcomes, especially in the short term, will arguably contribute to evidence on the model's reform potential. Since MHs have been implemented in different health systems, the next section will review outcomes from the model's implementation in Integrated Delivery Systems (IDS). Providers at this system level were early adopters of the model; therefore, outcomes from IDS will provide another delivery system level perspective on the relative effectiveness of the model's application.

\section{Improved Cost-Sensitive Outcomes from Integrated Delivery Systems (IDS)}

The focus of this section will be the Group Health Cooperative (GHC) in Seattle, Washington; Geisinger Health Systems (GHS) Proven Health Navigator (PHN) in Pennsylvania; and the Veterans Health Administration's (VA) Patient Aligned Care Team (PACT) initiative in the Midwest States. Because these providers were early adopters of the model, their outcomes are often referenced to support the model's viability and reform potential in the short run. GHC and PHN pilots began in 2006 by emphasizing primary care, chronic care management, and payment incentives for comprehensive chronic disease management. The available evidence suggests that GHC and PHN improved patient outcomes as well as reduced ED and inpatient care utilization and overall cost of care after case-mix and baseline-risk adjustments.

In GHC, quasi-experimental evaluations by Ralston et al. (2009) and Reid et al. (2009) found that pilot sites reduced in-person primary care utilization while overall primary care access rose 5 percent through secure phone and email 
communication. Health care utilization fell by 29 percent after 21 months, while quality of care remained the same. Primary and specialty care costs rose by $\$ 1.60$ and $\$ 5.80$ respectively on per-member-per-month (PMPM) basis, but the cost increases were offset by lower ED and IP use, which helped achieve an overall cost savings of \$14.18 PMPM across all intervention groups after risk adjustment. The improved outcomes, however, were statistically non-significant (Reid et al., 2010; Meyer, 2010). PHN expanded access to primary care services and restructured physician incentives as part of their reforms. The available evidence suggests that PHN achieved 28 percent and 8.1 percent reductions in IP admissions and ED use, respectively (Gilfillan et al., 2010; Steele et al., 2010). A follow-up evaluation suggested that cumulative costs after adjusting for prescription drug use fell by 7.1 and 4.3 percent, respectively, between 2006 and 2010 (Maeng et al., 2012). Most of the improvements in patient and cost outcomes from PHN were attained from improving care for patients in the Medicare Advantage Plan program.

VAs PACT covered Minnesota, North and South Dakota, Iowa, and Nebraska. This initiative emphasized primary care and chronic disease management and used HIT especially for chronic care coordination (Rice et al., 2010). The available evidence suggests that this initiative improved utilization, cost, and patient level outcomes. Since 2008 PACT has improved the management of congestive heart failure (CHF), chronic obstructive pulmonary disease (COPD), and diabetes, and it achieved 8 and 4 percent reductions in urgent care visits and acute care admissions, respectively (Rice et al., 2010). Total IP and ED use among all chronic disease 
patients fell by 27 percent, while the cost of care per chronic care patient fell by $\$ 593$ (Grumbach \& Grundy, 2010; Nielsen, Langer, Zema, Hacker, \& Grundy, 2012). The evidence from IDS corroborates the model's capacity to improve patient outcomes as well as ED and IP utilizations. The statistically non-significant results in the abovementioned studies, however, suggest the need for further studies on the relative effectiveness of the model's application, especially on the level of health risks in MHs and how that translates into utilization and other outcomes.

\section{Improved Cost-Sensitive Outcomes from State Programs}

Reviewing the available evidence from state-level programs will provide another layer and level of evidence to underscore the implementation of the model and its relative effectiveness. According to the National Academy of State Health Policy, MHs or comprehensive primary care initiatives are operational in most states around the country (Kaye, Buxbaum, \& Takach, 2011); while the evidence suggests improved ED and IP utilization, the evidence is mixed and varies across different states. Evidence from the following state programs will be reviewed: North Carolina, Vermont, Colorado, Minnesota, Oklahoma, Massachusetts, and Pennsylvania. These states were selected based on their early adoption of the Medical Home Model, use of state-developed or NCQA recognition standards, expansive use of the model by most providers across the state, use of multi payer provider payment incentives, and the availability of peer-reviewed research evidence.

North Carolina. Community Care of North Carolina (CCNC) focused on chronic care patients in Medicaid, expanded primary care services, and coordinated 
and integrated chronic disease management services for patients. The available evidence shows that this approach to care resulted in a cost savings of $\$ 974.5$ million from 2003-2008, \$382 million in 2010, and a PMPM savings of \$25.40 in 2010 (Grumbach \& Grundy, 2010). The largest savings accrued from reduced ED and IP utilization: ED use fell by 8 percent and 25 percent for pediatric patients with asthma and for all patients, respectively (Trapp, 2010); IP care for pediatric asthma also fell by 34 percent, while overall inpatient care fell by about 40 percent (Steiner et al., 2008; Nielsen, Langner, Zema, Hacker, \& Grundy, 2012). CCNC's strategy to screen and only enroll patients with elevated risks for multiple chronic physical and behavioral health conditions accounted for most of the improvements in ED and IP use and cost savings (Steiner et al., 2008; Trapp, 2010). The evidence from CCNC corroborates the argument that MHs can improve care and outcomes for chronic care patients.

Vermont. Since 2006, Vermont's Blueprint for Health (BPH) has reduced IP use per 1,000 admissions by 23.9, 15.3 and 39.7 percent at the three demonstration sites, respectively. ED utilization per 1,000 patient visits has also decreased by 33.8 , 18.9, and 2.8 percent at the three demonstration sites, respectively (Nielsen, Langner, Zema, Hacker, \& Grundy, 2012; Hester, 2010). Among BPH's core delivery strategies were engaging chronic care patients through continuous access to multidisciplinary teams as well as well-coordinated referrals. A focus group of chronic care patients revealed that their continuous access to providers resulted in their reduced ED use (Bielaszka-DuVernay, 2011; Hsiao, Knight, Kappel, \& Done, 
2011). While the evidence suggests that BPH focused on chronic care patients, the health-risk levels of patients in each panel of 20,000 was not reported in the study.

Colorado. In Colorado, a pre-post evaluation of the implementation of MHs showed the following improved outcomes: 18 percent reduction in acute IP utilization per 1,000 patient visits compared to the control group, and 15 percent reduction in ED utilization per 1000 patient visits (Raskas et al., 2012; Harbrecht \& Lisa, 2012). Cost of care in the intervention group decreased by $\$ 215$ per patient per year compared to the control group, while access to primary care services improved from 54 to 73 percent (Takach, 2011).

Minnesota. Minnesota's Health Care Home program, started in 2010, had the following improved outcomes: 39, 24, and 40 percent reductions in ED visits, IP admissions, and re-hospitalizations, respectively. Outpatient costs for patients using more than 11 medications, i.e. high health risk patients, were reduced by $\$ 1,282$ per patient (Flottemesch, Fontaine, Asche, \& Solberg, 2011). Average overall costs decreased by 8 percent (Nielsen, Langner, Zema, Hacker, \& Grundy, 2012).

Oklahoma. The available evidence suggests that Oklahoma's PCMH program reduced per capita cost per patient per year for Medicaid by \$29 from 2008 to 2010. Access to evidence-based primary care services, including screening for breast and cervical cancer also increased during the same evaluation period. Other access measures, including patient inquiries about same-day or next-day appointments, decreased from 1,670 inquiries to 13 within a year from 2009 to 2010 . PCMH enrollees responses to a survey in 2010 also suggested improvements in service 
delivery. Patients who responded that they always got treatment quickly, increased by 8 percent between 2008 and 2010 (Takach, 2011).

Massachusetts. The available evidence on the state's Patient-Centered Medical Home Initiative (PCMHI) suggest that there have been statistically significant improvements in various quality measures. Behl-Chadha, et al., (2017) suggested that PCMHI implementation in community health clinics resulted in improvements in 11 of 22 clinical quality measures, including chronic disease management, prevention and care coordination. A qualitative evaluation of the performance of clinics also showed consistent improvements on specific patient care metrics. The mean adjusted score for clinics on Self- management support (i.e., Providers support you in taking care of your own health) was 74 compared to 64 for comparator practices $(p<.001)$. Clinics also scored higher, compared to comparator practices, on Behavioral health integration measures (i.e., Providers pay attention to your mental or emotional health) (Cabral, Sefton, \& Anderson, 2013).

Pennsylvania. Evaluations of the PCCI initiative show that inpatient admissions decreased by $0.3,0.2$, and 0.2 in 2009, 2010, and 2011, respectively, while specialist visits were reduced by 12.3 and 10 per 1000 patients in 2010 and 2011, respectively. However, ED utilization and outpatient visits increased significantly (Neal, Chawla, Colombo, Snyder, \& Nigam, 2015). Friedberg, Schneider, Rosenthal, Volpp, \& Werner (2014) also suggested in their study that demonstration practices in PCCI improved quality of care after three years, but 
utilization and cost of IP, ED, and ambulatory care in demonstration practices did not decrease in the same period.

Summary of evidence. It can be broadly inferred from the above evidence on the application of the model for adult and pediatric patients, in IDS and from various state levels programs, that most $\mathrm{MH}$ programs, while using different verbiage to describe the model, emphasized the core elements of the model, namely comprehensive primary care, new ways for organizing health care delivery, stronger internal capacities for practices to provide effective care, and different incentives for health care delivery. The models had different patient focuses (adult, pediatric, or both) and were designed to provide care to different health risk patients, achieving varying levels of changes in ED and IP utilizations and expenditures. While the evidence suggests that chronic care patients can benefit from receiving care in Medical Homes, the reductions in ED and IP use and expenditures were mixed. For example, the evidence suggested that improvements in ED and IP use and costs were moderated when the programs focused on patients who were considered risky due to complex health conditions.

Starfield (2011) posited that "contrary to conventional wisdom, the main determinant of high costs of care is not the presence of chronic illness, but rather the combination of various types of illnesses or multi-morbidity" (p. 64). The comparatively higher utilization along with cost pressures from patients who have comorbid health conditions makes further insights into health risks necessary to understand the relative effectiveness of the application of the model. The focus of this 
research on evaluating chronic conditions-based health risks and their differential effects on ED and IP utilization is designed to provide another level of evidence for the relative effectiveness of the application of the model and the model's potential contribution to health care delivery reforms.

\section{The Importance of Health Risks to this Research}

It has been argued that how we pay for services directly determines the quality and quantity of services produced. This observation is important and applicable to health care delivery, especially to the growing use of risk-adjusted payments and physician profiling based on their patients. Evaluating PCPCHs based on their levels of health risks will help determine the relative effectiveness of the model's application to improve both health care expenditures and provider payment policies. The next section will introduce the concept of health risks and their importance to this research, evaluate the association between health risks and outcomes, and describe how this association helps frame this research perspective. This section will also discuss the use of the chronic disease burden measure in PCPCHs as a proxy measure for health risks in this research.

The role of risk selection and the effects of selecting healthy patients and dropping relatively sicker patients is empirically evident in health services literature (Newhouse, Buntin, \& Chapman, 1997; Thomas, Grazier, \& Ward, 2004; Maciejewski, Liu, Derleth, McDonell, \& Anderson, 2005). Provisions in the Affordable Care Act that stipulate equal access to care for patients who have preexisting conditions, combined with the current practice of health insurance payers 
profiling physicians and other health care workers based on the health risk levels of their patient panels for payment and other purposes, have created an environment in which tracking, prioritizing, and managing health risks have become important. Newhouse (1996a) observed that "regardless of how good risk adjusters become, we will never have a perfect formula, only better approximations of it” (p. 31).

Many independent studies have also questioned the efficiency and effectiveness of the numerous risk assessment and adjustment technologies in use (American Medical Association [AMA], 2009; Newhouse, 1996; Johnson, 2013; Horner, 2012). Despite these developments, many payers rely on current risk assessment and adjustment technologies for payment, provider profiling, and patient management. It can be argued that the questions surrounding risk assessment and adjustment methodologies make risk selection a viable option for some providers to achieve higher profiling scores, improve reimbursement, and subsequent profitability.

The focus of this research on evaluating the levels of health risks in PCPCHs is important because the evidence thus far is inconclusive. While some studies on MHs suggest that reductions in ED and IP use and health care expenditures are higher when $\mathrm{MH}$ interventions focus on chronic care patients considered high risk (Guy, Gunnarsson, Saynisch, Chawla, \& Nigam, 2013; Friedberg, Schneider, Rosenthal, Volpp, \& Werner, 2014; Warner, Duggan, Duey, Zhu, \& Stuart, 2013), other studies suggest mixed outcomes (Friedberg et al., 2014; Jackson \& Williams, 2015). In addition, while all states have implemented MHs, state programs are designed to focus on different patient populations. For example, North Carolina, Minnesota, and 
Colorado focus on chronic care patients, while Oregon's PCPCH is designed for all patients. Since the development of payment reforms to incentivize $\mathrm{MH}$ care has not kept pace with the model, and the fact that most of these payment systems are based on existing risk adjustment technologies that have problems identified above, evaluating the level of health risks in PCPCHs will provide information about the application of the model in the state and the relative effectiveness of the model's capacity to engage chronic care patients and improve ED and IP expenditures for such patients. The results of this evaluation can help improve payment incentives for recognized PCPCH providers.

\section{Association between Health Risks and Outcomes in Medical Homes}

The association between health risks and expenditure outcomes is implied because chronic care patients exert significant pressures on health care delivery through utilization (misuse, overuse, and underuse), which in turn contributes to higher health care costs. The association between health risks and outcomes is also empirically verifiable from the evidence on risk selection explained above (Newhouse, 1996; Johnson, 2013; Horner, 2012), and from MH implementation studies on ED, IP utilization, and health care expenditures for high chronic disease burden patients (Guy, Gunnarsson, Saynisch, Chawla, \& Nigam, 2013; Friedberg, Schneider, Rosenthal, Volpp, \& Werner, 2014; Werner, Duggan, Duey, Zhu, \& Stuart, 2013; Friedberg et al., 2014; Jackson \& Williams, 2015).

The associations between health risks and outcomes are evident in non-MH health care settings as well. Goetzel et al. (1998) and Ronaldo et al. (2013) evaluated 
health risk profiles among employees of large companies and found evidence suggesting that companies with lower health risk employees had lower health care expenditures. Evidence from the Diabetes Prevention program suggests that engaging patients who have higher risks for diabetes produces comparatively higher cost savings (Eddy, Schlessinger, \& Kahn, 2005; Russell, 2009; Goetzel, 2009).

The landscape of health care delivery underscores the association between health risks and outcomes. The evidence suggests that health care utilization and costs are disproportionately higher among patients who have various levels of chronic physical and behavioral health conditions. Figure 2.1 below illustrates the total health care expenditures in the United States in 2012, suggesting that fewer people used larger proportions of health care expenditures: 1 percent of the population is responsible for 22.7 percent of health care expenditures; 5 percent account for 50 percent; 10 percent for 66 percent; 25 percent for 86.7 percent; 50 percent of the population also account for 97.3 percent of health care expenditures; the remaining 50 percent of the population however, is responsible for only 2.3 percent of health expenditures. This distribution of health care expenditures in the United States has not changed significantly since 2012 . 
Figure 2.1

Total Health Expenditures for $2012=1.35$ Trillion

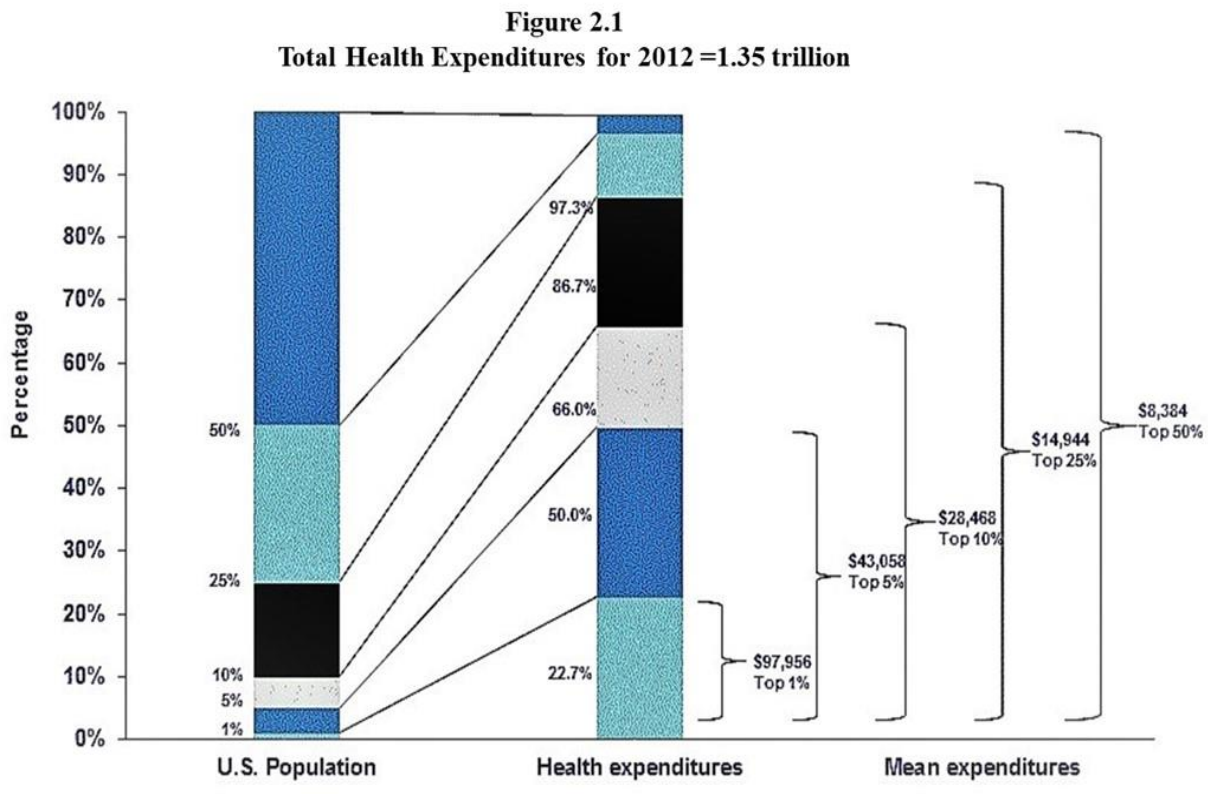

Source: Center for Financing, Access, and Cost Trends, AHRQ Household Component of the Medical Expenditure Panel Survey 2012

Based on the association between health risks, health outcomes and expenditures, and the expected benefits of MHs for all patients, especially chronic care patients, it can be argued that engaging a significant proportion of the patients who have high health expenditures in MHs, for example, the 50 percent of patients who use 97.3 percent of health expenditures could provide potential opportunities for improving health care delivery through providing comprehensive primary care services upstream while reducing downstream utilization of ED and IP and expenditures. Evaluating the level of health risks in MHs will provide important 
insights into the relative effectiveness of the model's application in the short term and help predict the model's viability and sustainability over the long term.

The evidence suggests that the trajectory of health care cost growth slowed after federal reforms, including $\mathrm{MH}$ reforms in 2010, but there is no consensus on the causes of the slowdown. While some studies postulated that the implementation of the Affordable Care Act may have contributed to the slowdown (Altman, 2015), others suggested a mix of factors that included economic slowdown (Getzen, 2014). Regardless, it can be argued that the sustainability of $\mathrm{MH}$ reforms in the long term will depend on the model's capacity to effectively manage chronic conditions and help reduce downstream pressure on ED and IP utilization and expenditures in the short term. In a resource-constrained environment, short-term improvements will be important for long-term support of the model. While short-term improvements in outcomes may not necessarily translate into long-term cost savings, short-term improvements will at least suggest the potential viability and sustainability of the model over the long term. The focus of this research on the application of PCPCHs will evaluate the short-term effects of health risks on improvements in ED and IP outcomes.

\section{Health Risks and Risk Assessment}

Health Services Research on health risks and risk assessments have traditionally analyzed demographic, administrative, and self-reported health status data to predict expenditures and patient outcomes. Demographic data broadly includes age, gender, family status, and residential location of patients (via zip codes). 
Administrative or claims data sources include prior expenditure and diagnosis codes or related groups and prescription use, while self-reported data use has focused on self-reported health status surveys. The validity of some of these data sources, however, has been questioned. For example, self-reported data is considered subjective, while the use of previous health care expenditure data tends to inflate the cost of services because future utilization and costs are based on previous utilization (Martin, Rogal, \& Arnold, 2004).

Health risks will be the primary independent variable of interest to this research. The relative effectiveness of PCPCHs will be assessed based on the levels of chronic conditions in PCPCHs. Conceptually, risk assessment uses claims data on patients' medical conditions and socio-demographic variables for predicting utilization and health care costs (Martin, Rogal, \& Arnold, 2004; Winkelman \& Mehmud, 2007). This research will create and use a chronic disease burden measure as a proxy health risk measure. Similar approaches to measuring health risks have been used in health services research (Hornbook \& Goodman,1996; Ash et al., 2000). The following 10 markers of chronic physical and behavioral conditions will be aggregated at the individual level to create a chronic disease burden measure: cardiovascular disease, chronic heart failure, coronary heart disease, chronic kidney disease, asthma/COPD, diabetes, obesity, affective disorder, other behavioral health, and schizophrenia. A weighted average chronic disease burden measure will then be calculated based on the average number of individuals and their proportional attribution for each PCPCH and comparator observation. 


\section{Theoretical Perspectives}

Health care delivery in the country is considered a 'market-based delivery system.' Among the assumptions of a market-based system are the availability of perfect information for all economic agents to make informed decisions and the efficient allocation of health care resources based on demand and supply, which results in efficient allocation of resources and effective outcomes. The current health care delivery system, however, lags most advanced countries on the main benchmarks of efficiency and effectiveness (Commonwealth Fund, 2015). Fragmentation in how health care delivery is organized has contributed to a complex delivery system environment that exerts pressure on health care costs, quality (overuse, underuse, misuse, and high error rates), resulting in a growing disease burden and declining patient outcomes.

Various reform initiatives including MHs were therefore implemented to help improve the organization, delivery, and outcomes of health care. However, it can be argued from a systems perspective that the relative effectiveness of ongoing reforms, including MHs, may depend on changes to existing incentive systems in the delivery system environment to foster cooperation among the various agents (payers, providers, and patients), which in turn will lead to improved care coordination, integration, and outcomes. For example, while all agents in a market-based system are assumed to have 'perfect information' to enable self-interest-based decision making, this is not the case in the current health care delivery environment. Asymmetric information in the environment suggests that some patients may not be 
receiving care in PCPCHs because they do not have information or incentives to selfenroll in PCPCHs. The theoretical perspectives of this research, Risk Selection in health care services delivery and Complex Adaptive Systems (CAS), provide insights into some environmental factors that could affect the engagement of patients whose health risks drive health care utilization and expenditures in PCPCHs.

Risk selection. The economic theory of Risk Selection posits that health risks are heterogeneous, unevenly distributed in populations, and un-priced (Newhouse, 1996a). The un-priced risk heterogeneity makes providing care for relatively healthy patients more financially rewarding than patients with various chronic physical and behavioral health conditions. Rational economic agents such as providers and payers tend to select 'good' risks based on the health conditions of patients and their potential health care utilization and cost of care. Risk Selection persists because the nature of health risks creates transaction costs in separating and pricing health risk types. Most payers, therefore, use the average expected cost approach to reimbursement (Newhouse,1996b).

This approach suggests that providers who treat patient panels with higher than average health risks may operate at a financial disadvantage because they will be reimbursed based on the average expected cost, which may be less than the cost of treating such patients. The average cost approach to reimbursement, furthermore, has implications for the use of comprehensive payments for MH care, especially how reimbursements for provider teams of chronic care patients are structured to incentivize provider teams to effectively coordinate and integrate care for these 
patients. The nature of health risks and the evidence on Risk Selection in health care service delivery has been well documented (Newhouse, Buntin, \& Chapman, 1997; Ettner, Frank, McGuire, \& Hermann, 2001; Kansagara et al., 2011).

The lack of access to health care information for some agents, especially patients and some providers, make Risk Selection likely in the current delivery system environment. The second theorem of optimality posits that information asymmetry contributes to market imperfections and makes risk selection and 'cream skimming' possible (Arrow, 1963; Newhouse, 1996; Reinhardt, 1998). In a market approach to delivering health care, information asymmetry creates imbalances in the agency relationships among patients, providers, and payers (private, public, and individuals) and potentially contributes to uncertainty, Risk Selection, and ineffective health care delivery (Newhouse, 1996a; Williamson, 2005). For example, while MHs are designed to benefit chronic care patients, most of these patients do not have sufficient information to determine their health conditions and the benefits of enrolling in MHs. The lack of transparency in the pricing of medical care and the alignment of incentives in health care production can also affect patients' capacity to determine the true cost and benefits from enrolling in MHs. This information gap increases the likelihood that the interests of patients, providers, and payers may not be aligned in health care production, therefore increasing the likelihood of patient selection and the avoidance of high risk patients.

Risk Selection is further possible because the health care production environment allows heterogeneous firms (providers and payers) with different 
production costs to use strategies such as offering different products, employing different staff, and locating in different communities to attract relatively low risk patients (Ma, 1994; Ellis \& McGuire, 1986). Even though PCPCHs and all health care providers are ethically compelled to do no harm (i.e. non-malfeasance) while providing health care services, it can be argued that, all things being equal, the economic environment and incentive structures will influence providers' agency relationships regarding the types of patient risks to attract.

PCPCH clinics that enroll higher-than-average health risk patients while collecting average-cost reimbursements may not be economically viable or sustainable in the short- and long-term. As a result, the potential capacity of PCPCHs to reduce the overuse and misuse of ED and IP and their associated expenditures may be minimized. Risk Selection can be positive or adverse. Positive selection occurs when providers can attract relatively healthy patients whose health risks are lower than the average Medicaid or community risk. Adverse Risk Selection, on the other hand, occurs when providers can attract relatively sicker patients who have higher health risks than the average Medicaid or community risk (CMS, 2003). Since containing rising health care costs is a goal of the Triple Aim, evaluating the levels of health risks in PCPCHs will help determine whether PCPCHs are enrolling the types of patients whose risks drive utilization and expenditures.

Complex Adaptive Systems (CAS). Plesk (2001) defined CAS as a "collection of individual agents who have the freedom to act in ways that are not always predictable and whose actions are interconnected such that one agent's actions 
change the context for other agents" (pp. 312-313). CAS systems are comprised of the following elements: open systems with feedback loops, operating far from equilibrium, actions that are embedded in the context of their histories, continuous interaction with their environments, and interactions that often result in nonlinear effects (Holden, 2005). Crabtree et al. (2011), Holden (2005), Crabtree, McDaniel, Miller, \& Stange (1998), and Plesk (2001) all consider health care delivery broadly, and primary care practices as CAS, because the elements in their environments influence health care production and outcomes. Considering PCPCHs as CAS provides another theoretical lens for analyzing the relative effectiveness of the application of the model, specifically how the complex health care delivery system environment influences health risk levels in PCPCHs and reductions in ED and IP expenditures.

Among the PCPCH agents whose actions and interests are interconnected during health care production include patients, providers, and payers. Conceptually, health care production in PCPCHs emphasizes patient-centered relationships that are formed around multidisciplinary care teams. Optimum health care production depends on engaging all patients, including patients who have chronic conditions; however, asymmetric information in the health care production environment, along with the profiling of providers and paying for health care services, create barriers for providing care to chronic care patients in all settings, including PCPCHs, and to incentivizing selection. 
A complex and adaptive delivery environment that is characterized by information asymmetry could affect the capacity of chronic care patients to evaluate multiple components of their health care: the benefits of enrolling in PCPCHs, the likelihood that the composition of PCPCH patient panels will reflect the health risks levels in their communities, and PCPCHs' capacity to reduce the inappropriate use of ED and IP and expenditures. One example of the potential effects of information asymmetry is the structural and delivery system problems a Massachusetts woman encountered in her quest to compare provider prices to inform her choice of provider for vaginal delivery (Bebinger, 2014). This example is arguably repeated in many patient encounters across various levels of the delivery system, and it reflects structural problems in the market-based approach to health care production that can have consequences for patients' agency relationships, and potentially the outcomes of care in PCPCHs.

Health care delivery has gone through several stages of reforms and transformations. Crabtree et al. (2011) observed from historical evidence on primary care transformation that improving outcomes of care may only be achieved from "major changes to the interdependent relationships among agents" (p. S30). By design, $\mathrm{MH}$ reforms focus on one level of the delivery system; the capacity of the model to create broad systems changes and improve outcomes may be limited in the current delivery system environment and relationship among agents. While the PCPCH model can in theory help reduce the overuse and misuse of ED, IP, and expenditures, the relative effectiveness of the model will depend on coordinating and 
integrating care for patients, especially those with various levels of physical and behavioral health conditions. Theories on CAS and Risk Selection, however, suggest there could be environmental factors that can affect the application of the model and the expected outcomes.

Summary of theoretical perspectives. It can be inferred from the theories of Risk Selection and CAS that health care incentives may not be aligned to engage chronic care patients or change the paths of patient populations who have higher ED and IP utilization. The evidence from PCPCH implementation thus far suggests that clinics are operational in different communities and by different payer groups. Because different patient populations and communities have different health risk levels, PCPCHs patient panels likely contain different health risks.

In a delivery system environment characterized by asymmetric information, CAS suggests that PCPCHs' health care production may not attain equilibrium if health risk levels in their patient panels are lower than the health risk levels in the communities they operate. Reductions in ED and IP use and expenditures may also not be optimum. This current health care delivery environment, therefore, makes opportunism a potential threat to the relative effectiveness of the application of the PCPCH model. Williamson (1979) defined opportunism as “a variety of self-interest seeking behavior that includes self-interest seeking with guile" (p. 234). According to Williamson (1979), opportunism will arise in a market environment characterized by high transaction costs and asymmetric information. To reduce the effects of 
opportunism, Williamson proposed the use of markets and hierarchies as governance mechanisms to safeguard the "integrity of transactions" (p. 22).

While the use of various comprehensive payment incentives for PCPCH care is designed to incentivize clinics and providers to engage high-risk patients who will need and benefit from PCPCH care, the effectiveness of these payments will depend on how each provider team member engages high health-risk patients, and how that translates into the allocation of proportional payments for provider team members. The evidence on Risk Selection and wasted capacity in health care services delivery suggests challenges to the payment reforms for incentivizing patient engagement. Evaluating the health risk levels in PCPCHs will help determine whether PCPCHs are engaging the types of patients who have various levels of chronic physical and behavioral health conditions.

The theories of this research do not conclusively prescribe specific health-risk distributions in PCPCHs or reductions in the overuse and misuse of ED and IP utilization and expenditures that will be achieved by PCPCHs. However, the theories suggest that if reducing or containing health care costs is a goal of ongoing PCPCH reforms, then evaluating how people with different health conditions are distributed throughout PCPCHs will provide information about the contributions of the model's application to reduce ED and IP expenditures.

\section{Grand Summary of the Evidence}

Medical Home variants are being implemented around the country as part of broader delivery system reforms. In Oregon, PCPCHs are an important element of 
health care transformation in the state to achieve the Triple Aim. Proponents of Medical Home reforms argued that the model's emphases on comprehensive primary care, care coordination and integration for chronic disease management, and the use of provider teams across the care continuum will accomplish the following: improve care for all patients, especially those with chronic physical and behavioral conditions; reduce the overuse, misuse, and underuse of ED and IP; and contain rising health care costs. However, it can be inferred that the model's capacity to improve the above outcomes will depend on enrolling patients who have comparatively higher health care utilization because of their chronic physical and behavioral health conditions.

A review of the evidence on the model's implementations thus far suggests mixed evidence for the capacity to reduce ED and IP use, expenditures, and health care costs. The evidence suggests that health risks are important and have varying effects on outcomes. The model is designed to provide upstream services to engage chronic care patients in ambulatory settings and change the paths of such patients from utilizing expensive ED and IP care; however, Risk Selection and the misalignment of incentives in health care delivery suggest that providers may be inclined to select healthy patients. In addition, patients who have higher risks may not enroll into MHs because of asymmetric information in the complex health care delivery system environment. This research proposes that the integration of primary care and CCM, and the coordination and integration of care for patients in PCPCHs, are necessary but insufficient for reducing ED and IP use and expenditures. In the short term, the composition of patients who have chronic physical and behavior 
health conditions in $\mathrm{PCPCH}$ panels will be important for reducing the overuse and misuse of ED and IP utilization and expenditures. The implementation of PCPCHs for Medicaid patients and all the state's population provides opportunities for evaluating the health risk levels of PCPCHs and their associations with reductions in ED and IP expenditures in the short term. Based on the history of the model, evidence on the application of the model thus far, and the specific theories of this research, the following research questions and hypotheses emerged.

\section{Research Questions and Hypotheses}

This dissertation will explore the following three questions:

1. What is the average chronic disease burden of PCPCHs, and how does their average chronic disease burden compare to the communities PCPCHs are in pre-post PCPCH recognition?

It is hypothesized that primary care clinics that become PCPCHs will initially have chronic disease burdens equal to their community comparators, but the chronic disease burden in PCPCHs will be higher than their communities after recognition, based on the expectation that PCPCHs will enroll more chronic care patients.

2. How do primary care expenditures change based on the chronic disease burden of PCPCHs?

It is hypothesized that primary care expenditures will be higher after $\mathrm{PCPCH}$ recognition, for high chronic disease burden PCPCHs, based on the expectation that PCPCHs will provide more primary care services to high chronic disease burden patients who will need and use more primary care services. 
3. Do PCPCHs that engage more high chronic disease burden patients have more reductions in ED and IP expenditures?

It is hypothesized that PCPCHs will achieve higher reductions in ED and IP expenditures after $\mathrm{PCPCH}$ recognition, based on the expectation that patients who have high chronic disease burden will have greater response to PCPCH services, such as comprehensive primary care, including care coordination, integration, and continuity, which will translate into reductions in the inappropriate (overuse and misuse) of ED and IP services.

Answers to these questions will contribute to the knowledge base and evidence for the model's application and relative effectiveness. This research will primarily be exploratory because the introduction of the model and evidence for the model's application are emerging fields of study. The literature on health risks and Risk Selection suggests that both concepts are complex in their application to health care services research (Newhouse, Buntin, \& Chapman, 1997). Plesk's (2001) argument that changes in health care tend to lie in "zones of complexity" and often results in nonlinear outcomes applies to this research. 


\section{Chapter 3: Research Methods}

This chapter describes the quantitative research methods for testing the proposed hypotheses and answering the research questions posed in Chapter 2. A natural experimental research design is used to estimate the policy effects of the application of the Patient-Centered Primary Care Home (PCPCH) model, specifically, changes in the chronic disease burden levels in PCPCHs and their association to PC, ED, and IP utilization. This chapter is organized according to the following main headings: Research Design, Sources of Data, Study Population, Data Attribution Methods, Aggregate PCPCH and Comparator Measures, Dependent Variables, Independent/Case-Matching Variables, Empirical Methods, Evidence Base for Empirical Methods, Difference-in-Difference Models, Statistical Analysis, and Limitations of Research Methods.

\section{Research Design}

This research is designed as a natural experiment. Opportunities for natural experimental studies arise when policy or program changes create disparate impacts on different population groups. The policy or program effects can be evaluated on the 'naturally' formed groups such as those who are affected by the changes (i.e. treatment groups) and those who are unaffected (control groups). Exogenous variation in natural experiments is measured based on changes to the treatment and control groups (Meyer, 1995). Oregon's PCPCH program was introduced in October 2011 as part of a state-wide delivery system transformation (HB 2009) to promote the Triple Aim, which includes reducing or containing health care costs. Based on the 
evidence of association between health risks and health care costs, this research evaluated the levels of health risks in PCPCHs to determine the model's capacity to achieve the aim of reducing health care costs through improved PC, ED, and IP utilization. This natural experimental study compared the recognition of some primary care clinics and providers as $\mathrm{PCPCHs}$ to non- $\mathrm{PCPCH}$ primary care clinics.

Health risks are important to this research. A chronic disease burden measure was developed and used as a proxy health risk measure for this research. The chronic disease burden measure was based on the following 10 markers of chronic conditions: cardiovascular disease, chronic heart failure, coronary heart disease, chronic kidney disease, asthma/COPD, diabetes, obesity, affective disorder, other behavioral health, and schizophrenia. The policy effects of the introduction of PCPCHs were measured by comparing the average chronic disease burden in PCPCHs (intervention group) and non-PCPCHs (control group) to determine the differences in chronic disease burden and the average expenditures for PC, ED, and IP by chronic disease burden levels pre-post PCPCH recognition. Data on primary care patients in 2011 was used for constructing the pre-policy period while the post-policy period comprised data on the first wave of PCPCH recognition from 2012 to September 2014. This research design was implemented through difference-in-difference methods, which will be explained later in this chapter.

\section{Sources of Data}

Data from Oregon's All Payer All Claims (APAC) data system, which includes claims and eligibility files, and data from the PCPCH attestation process for 
recognizing primary care clinics as PCPCHs were utilized for this research. Oregon's APAC data system provided standardized data on health care utilization, which included pharmacy claims, patients' de-identified demographic information, and payer information from private, public and third-party health plans and payers (Love, Custer, \& Miller, 2010).

\section{Study Population}

The study population comprised patients receiving primary care in Oregon. Primary care was the level of analysis while PCPCH clinics were the unit of analysis. To effectively evaluate the policy effects of the application of PCPCHs, this study utilized quarterly data on primary care patients from October 2011 through September 2014. The pre-policy implementation period was based on data from October 2010 to September 2011, while the post-policy implementation period utilized data from October 2011 to September 2014. Selection was based on patients who had at least one primary care visit from a designated PCPCH or a community comparator practice within the study period. Using this date span helped capture periods before and after the introduction of PCPCHs and minimized the policy implementation effects by excluding data from three months before and after policy implementation. Due to the span of data that was available, the research and its findings will only cover the first wave of PCPCH attestations up to September 2014. 


\section{Data Attribution Methods}

As part of attribution in this research, patients and providers were assigned to PCPCH and community comparators. Patients were attributed to PCPCHs on a proportional basis. As part of this measure, the number of a patient's primary care visits and the total $\mathrm{PCPCH}$ visits were calculated and weighted to determine the proportional $\mathrm{PCPCH}$ visits per patient. The proportional attribution of patients to PCPCHs helps account for the care of patients who received care from multiple PCPCH providers per period (Higgins, Zeddies, \& Pearson, 2011; Lewis, McClurg, Smith, Fisher, \& Bynum, 2013; Harbrecht \& Latts, 2012). The attribution of primary care clinics and providers as PCPCH was based on a cross-walk key comprising the providers' ZIP codes from their national provider identifier and from $\mathrm{PCPCH}$ attestation data from the state's recognition data. Primary care clinics that applied for and were recognized by the state as PCPCHs during that period were attributed accordingly as PCPCHs. Primary care clinics that applied for but were not recognized were dropped from the study. These attribution methods helped identify PCPCH and non-PCPCH patients, create PCPCH aggregate measures, and construct the community comparators through a case-matching approach like propensity score matching.

Development of the chronic disease burden measure. Based on the design of this research to evaluate the general distribution of health risks in PCPCHs and their relationship to selected outcome measures, a simple chronic disease burden measure was developed and used as a proxy health risk measure for this research. 
Previous health services research (Ash \& Ellis, 2012; Hornbook \& Goodman, 1996; Ash et al., 2000) have used similar approaches - using markers of chronic conditions to evaluate the performance of primary care clinics and to adjust primary care provider payments. To develop the chronic disease burden measure, primary diagnosis data for each study subject was used to identify the presence of the following 10 conditions: cardiovascular disease, chronic heart failure, coronary heart disease, chronic kidney disease, asthma/COPD, diabetes, obesity, affective disorder, other behavioral health, and schizophrenia. A binary condition marker was created with value of 1 if a patient had at least one claim with a primary diagnosis consistent with the 10 conditions. The following ICD-9 diagnoses codes were used to identify each condition: Chronic Health Failure: 428.00-428.99; Chronic Heart Disease: 420.00-420.99, 410.00-414.99,429.20-429.09; Chronic Kidney Disease: 585.00585.99; Cerebrovascular Disease: 438.00-438.99; Chronic Obstructive Pulmonary Disease(COPD): 490.00-490; Diabetes: 250.00-250.99; Obesity: 278.00-278.09; Schizophrenia: 295.00-295.99; Affective Disorders: 296.00-296.99; and, Other Behavioral Health Conditions: 297.00-312.99.

The 10 markers of chronic conditions were summed at the individual patient level to develop an individual chronic disease burden score. The markers for the three behavioral health conditions (schizophrenia, affective disorder, other behavioral health) are independent; therefore, the maximum aggregate score for the 10 conditions was 8 . An aggregate average chronic disease burden measure was then calculated for each PCPCH and community comparator observation in each of the 16 
quarters of study data (Q4 2010 to Q3 2014) based on the number of quarterly individuals weighted by their proportional attribution to each PCPCH or comparator.

These measures of average chronic disease burden were used to assess trends in PCPCHs over time and, in comparison to their community comparators. Among PCPCH observations, the quarterly chronic disease burden measures were further aggregated and averaged to construct an average profile across all study years. This calculation was used to stratify PCPCHs into high and low chronic disease burden groups based on the median of the PCPCH chronic disease burden measures.

This stratification was used for this research and analysis in two ways: to compare outcomes for high and low chronic disease burden PCPCHs, and to compare outcomes for high and low chronic disease burden PCPCHs to their respective community comparators. Specifically, the average changes in the dependent variables for research questions 2 and 3 were measured from three different perspectives: comparing high and low chronic disease burden PCPCHs, comparing low chronic disease burden PCPCHs to their respective chronic disease burden community comparators, and comparing high chronic disease burden PCPCHs to their respective chronic disease burden community comparators. The different levels of comparison helped assess whether the comparison of high-versus-low chronic disease burden PCPCHs did not simply reflect differences in the PCPCHs' communities.

\section{Aggregate PCPCH and Comparator Measures}

The aggregate measures in this research were based on case-matching the independent variables (the selection of which is explained below): age, gender, health 
insurance payer type, and ZIP code of patients. As part of the matching process, observations from each independent variable were aggregated and weighted to create PCPCH aggregate measures. The weighted PCPCH measures were then used to identify and construct community comparators. To ensure that primary care patients in the community comparator groups reflected the types of patients the $\mathrm{PCPCH}$ served, the independent variables were used in a case-matching process to identify community comparators (non PCPCH clinics).

As part of this process, data on each independent variable was aggregated and weighted to ensure that the elements, dimensions, and proportions of the PCPCH and its community comparator were the same. Doing so improved the comparison process and ensured that PCPCHs were analyzed against comparators that had similar characteristics and reflected both the types of patients the PCPCHs served and the communities where the PCPCHs operated. Community comparators comprised primary care clinics and providers in the state that are not recognized as PCPCHs. Clinics that applied for PCPCH recognition but were unsuccessful in their application as well as clinics preparing to apply or currently going through $\mathrm{PCPCH}$ recognition were excluded from the comparator groups to minimize the effects of confounding.

\section{Dependent Variables}

The dependent variables of this research were the following: chronic disease burden of PCPCHs and their comparators, primary care expenditures by PCPCHs and their comparators, average ED utilization by $\mathrm{PCPCHs}$ and their comparators, and average IP utilization by PCPCHs and their comparators. Individually, each outcome 
variable helps evaluate a specific element of the application of the model, and collectively they provide insights into the application of the model in the state. Proponents of the PCPCH model argued that its implementation could improve primary care for patients in ambulatory settings and reduce the reliance on and utilization of ED and IP for primary care related health needs (Peikes, Dale, Lundquist, \& Genevro, 2011; Gilfillan et al., 2010; Reid et al., 2010; Reid et al., 2009; Rittenhouse, Thom, \& Schmittdiel, 2010; David, Gunnarsson, Saynisch, Chawla, \& Nigam, 2013). Embedded in this argument is the expectation that the utilization of some primary care services will increase in PCPCHs, which will translate into reduced ED and IP utilization and expenditures because some of the health needs that drive patients to use ED and IP will be appropriately addressed through comprehensive primary care upstream in ambulatory settings. However, other research suggests weak associations between implementing the model and improved ED, IP, and health care costs (Friedberg, Schneider, Rosenthal, Volpp, \& Werner, 2014; Werner, Duggan, Duey, Zhu, \& Stuart, 2013).

Based on established associations between health risks and outcomes (Conwell \& Cohen, 2012; AHRQ, 2011), this research proposes that the levels of chronic conditions in PCPCHs based on the chronic disease burden measure could determine the model's capacity to improve ED and IP utilization. The association between health risks and outcomes makes the chronic disease burden levels in PCPCHs important to the model's reform potential. The evidence suggests that chronic care patients usually utilize health care services disproportionately, especially 
ED and IP care. This association has been confirmed by research (Wolff, Starfield, \& Anderson, 2002; Johnson et al., 2012). Ascertaining chronic disease burden in PCPCHs and their effects on utilization of ED, IP, and PC will help determine the relative effectiveness of the application of the model and reform potential.

Chronic disease burden of PCPCHs and their comparators. Based on the expected association between chronic disease and utilization, this research developed and used the average chronic disease burden measure as a dependent variable for a step-wise evaluation of the application of PCPCHs to help answer the three research questions. As part of the first research question, the chronic disease burden measure of PCPCHs was compared pre-post PCPCH recognition to their community comparators (practices within their respective PCPCH communities) to determine whether PCPCHs are changing the types of patients they served based on the levels of chronic disease burden in PCPCHs after clinic recognition. To help answer Research Questions 2 and 3, the average chronic disease burden measure for PCPCHs was stratified into high and low chronic disease burden groups based on the median chronic disease burden measure, which was used to evaluate associations between high and low chronic disease burden PCPCHs and their utilization and expenditures for PC, ED, and IP.

Primary care expenditures by PCPCHs and their comparators. Primary care (PC) expenditures by chronic disease burden levels (high versus low) were determined and compared pre-post $\mathrm{PCPCH}$ recognition between $\mathrm{PCPCH}$ and their community comparators to determine the differences in primary care use by high and 
low chronic disease burden groups. Comparing PC expenditures between high and low chronic disease burden groups in PCPCHs helped capture PC expenditures by each chronic disease burden group and determine whether high chronic disease burden patients used more primary care services. This comparison is important because engaging patients by providing them with more appropriate primary care services upstream is expected to reduce the misuse and overuse of ED and IP utilization downstream. Variations in primary care expenditures by the high and low chronic disease burden PCPCHs and their community comparators will provide insights into the relative effectiveness of the application of the PCPCH model.

Average ED and IP expenditures by PCPCHs and their comparators. The expenditures for ED and IP by high and low chronic disease burden PCPCHs were evaluated separately. As part of this process, the high and low chronic disease burden groups for PCPCHs, as well as the high and low chronic disease burden PCPCHs and their respective community comparators, were compared pre-post $\mathrm{PCPCH}$ recognition to their respective comparators to determine the differences in ED and IP expenditures by the different chronic disease burden groups and whether high chronic disease burden PCPCHs achieved more savings from engaging more high chronic disease burden patients. While ED and IP use are important elements of effective health care delivery, higher levels of ED and inpatient expenditures are considered ineffective (i.e. overuse and misuse). The evidence suggests that some ED and IP utilization can be avoided through effective upstream care in ambulatory settings (Berwick, Nolan, \& Whittington, 2008; Ansari, 2007; Peikes, Dale, Lundquist, 
Genevro, \& Meyers, 2011). Because the overuse of ED and IP care exerts upward pressure on health care costs, ED and inpatient utilization have become important outcome metrics for policy decisions on the relative effectiveness of the application of PCPCHs (Cooley, McAllister, Sherrieb, \& Kuhlthau, 2009).

\section{Independent/Case-Matching Variables}

The following independent variables will also be utilized for this research: age, gender, health insurance type, and ZIP codes of PCPCH clinics. These independent variables will be aggregated, weighted, and used to construct PCPCH comparator groups for the comparative analysis of the application of PCPCHs. This matching approach is similar to propensity score matching and will help compare PCPCHs to their respective community comparators based on similar patient characteristics. PCPCHs and their communities will not be matched on the chronic disease burden measure. Instead, the chronic disease burden measure will be stratified to high and low chronic disease burden groups and used as explained above to evaluate PC, ED, and IP utilization and expenditures by PCPCHs and their respective comparator groups.

Age. The natural cycle of human aging, morbidity, and mortality makes age a relevant variable in chronic disease burden (health risks) analysis. The evidence supports the association between aging, health care utilization, and costs (Wolff, Starfield, \& Anderson, 2002). About 90 percent of Medicare spending is incurred on elderly patients because of their chronic conditions (AHRQ, 2011). Until recent improvements in predictive modeling on health risks as a metric for profiling 
providers and patients, age was the primary variable for such predictions (Center for Health Program Development and Management, 2003; Winkelman \& Mehmud, 2007). The evidence of association between aging, chronic disease burden, and outcomes is strong. Wolff, Starfield, \& Anderson (2002) found associations between aging, the risks of chronic conditions and higher health care expenditures. Higher utilization and health expenditures have also been found among younger age groups. Neff, Sharp, Muldoon, Graham \& Myers (2004) found that chronic conditions in pediatric populations in Washington State accounted for disproportionately higher health care expenditures. Shi and Lu (2000) suggested that children between 0-15 years and children who are black had higher rates of hospitalizations. The National Hospital Ambulatory Medical Care Survey also found that about a quarter of the 115.3 million ED utilizations in 2005 were by pediatric patients (Nawar, Niska, \& $\mathrm{Xu}, 2007)$. The above evidence supports the inclusion of age as a predictor of chronic disease burden and utilization. Including age as a predictor and matching variable will help account for some of the variation in health outcomes.

Gender. In accordance with most studies on health risks, this study will include and account for the effects of gender on utilization and outcomes because the evidence suggests that health risks, utilization, and health care costs vary by gender. For example, while males have comparatively higher rates of preventable hospitalizations for primary care related conditions, females have comparatively higher health care utilization than men (Culler, Parchman, \& Przybylski, 1998; Zaslavsky \& Epstein, 2005). Most health insurance rates accordingly vary by gender 
(Center for Health Program Development and Management, 2003; Winkelman \& Mehmud, 2007). Gender will also be used as a case-matching variable for community comparators.

Health insurance type. The availability and affordability of health insurance are important predictors of access to health care services, especially in market-based delivery systems. Starfield and Shi (2004) posited that providing access to health insurance can improve the effectiveness of MHs through reduced ED and IP utilization because most people without health insurance tend to utilize ED for their primary care needs. Billings, Anderson, and Newman (1996) found that low-income patients in poor health had high rates of ED and preventable hospitalization because of their health insurance status.

In Oregon, Wallace, McConnell, Gallia, and Smith (2008) found that increasing the copay for some population groups affected their access to care as well as their use of ED and IP care and other health services. Saha, Solotaroff, Oster, and Bindman (2007) also found that access to the Oregon Health Plan generally resulted in reduced ED and inpatient utilization. Among other studies that corroborate the association between health insurance status and the use of ED and IP care include Kogan et al. (1995), Hoffman and Paradise (2008), and Murty, Beglay, \& Swint (2011). Based on the above evidence, health insurance type was included as a predictor and case-matching variable for creating community comparators.

ZIP codes of PCPCHs. The community location of PCPCH clinics is important to this research because of evidence suggesting that health, health care 
utilization and outcomes differ in various geographic locations and are affected by various social and economic factors as well (Healthy People, 2020). Figure 3.1 below, broadly capture the social and economic factors that tend to differ by geographic location and have been found to significantly determine levels of access to health care services and the quality of health.

\section{Figure 3.1}

Social Determinants of Health \& Equity

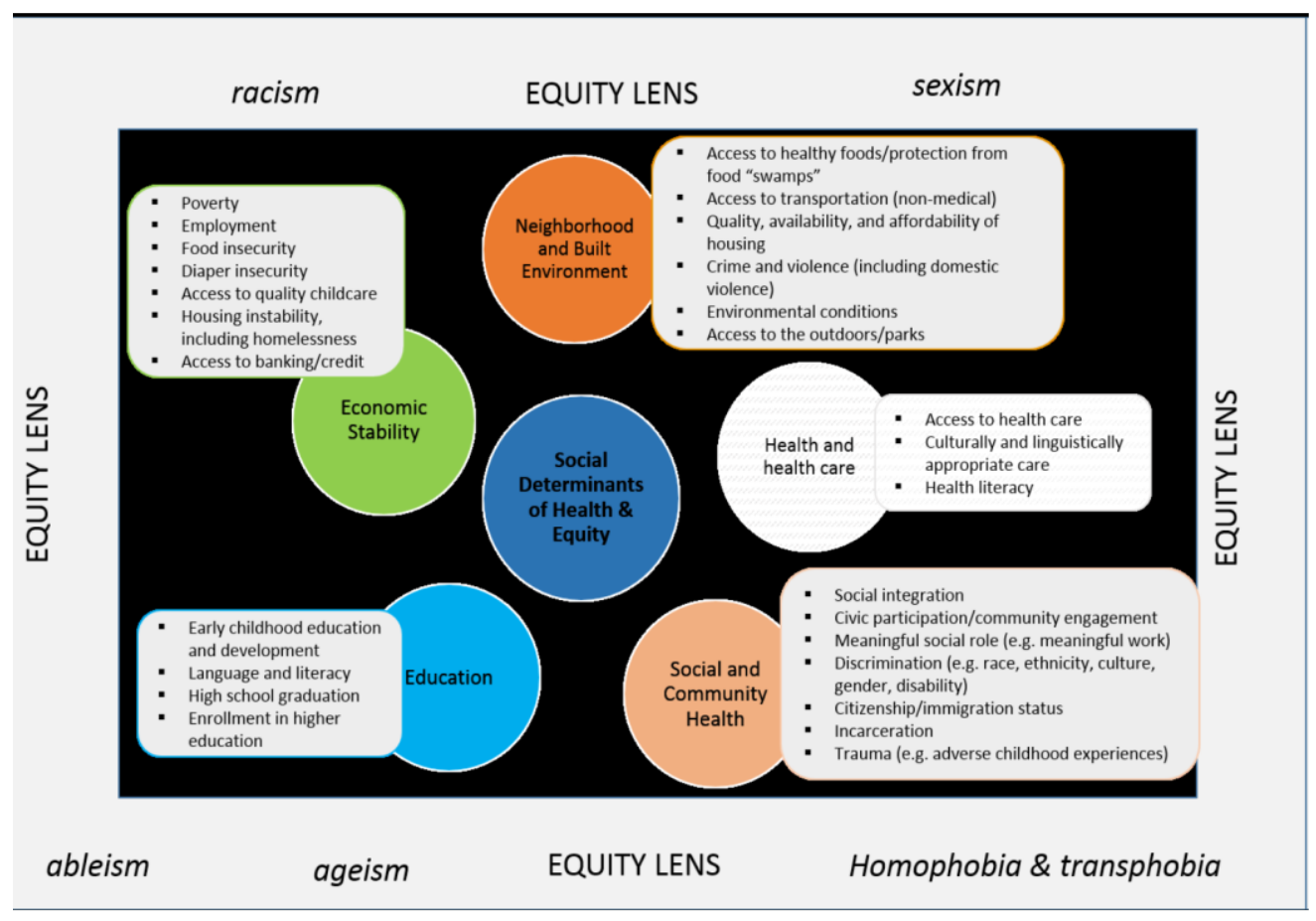

Source: Oregon Medicaid Advisory Committee (2017)

The Healthy People 2020 initiative defines the determinants of health to include the geographic conditions into which people grow, live, work and age, these conditions include housing, education, employment, access to health care, transportation, and neighborhood environments. There is growing evidence 
suggesting that chronic conditions and their associated health risks and outcomes vary by geographic locations (Starfield, Shi, \& Macinko, 2005). Goodman et al. (2003) and DiLia (2003) found that preventable hospitalizations differed by geographic location. Various international studies corroborate that access to primary health care services in some communities significantly improved utilization and outcomes. For example, a United Kingdom study found that timely access, measured by the travel time to a primary care provider, was associated with reduced "self-referred ED visits" (Cowling et al., 2013).

Based on the above evidence that health outcomes varied by geographic location and access to primary care services, the ZIP code of patients will be utilized for case-matching to construct community comparators. The Primary Care Service Area (PCSA), which includes ZIP codes, has been previously used for measuring community effects in health care, but this study will use only ZIP codes because they are sufficient to create PCPCH comparators.

\section{Empirical Methods}

The natural experimental design relies on difference-in difference (D-in-D) models to empirically estimate the policy effects of the introduction of PCPCH. The policy effects are measured as the pre-post DD in the chronic disease burden levels of patients, the use of primary care services by chronic disease burden groups (high and low), and ED and IP utilization by chronic disease burden between PCPCHs and nonPCPCH comparators. The use of DD helps accomplish two specific research objectives: (1) measure specific outcome differences from the introduction of 
PCPCHs from two different time periods (pre-post), and (2) compare the outcome differences between PCPCHs and non-PCPCH comparators (Buckley \& Shang, 2003). D-in-D estimation is often used for measuring exogenous variation from policy or program changes. At the core of D-in-D methods is the source of exogenous variation and the interaction effects over time (Bertrand, Duflo, \& Mullainathan, 2001).

The aggregated PCPCH observations were used in a case-matching process to construct comparator groups as explained above. This process made patients in PCPCHs and their community comparators (non-PCPCHs) similar. As part of the process, each $\mathrm{PCPCH}$ practice in the study sample was matched by a national provider identifier (prov_npi) to a comparator practice that was similar in terms of patients' age, gender, health insurance type, and ZIP code of practice. The chronic disease burden measure was organized and used differently (as described above) to determine whether PC, ED, and IP expenditures differed based on the chronic disease burden of PCPCHs and their community comparators.

Like studies that have used control groups, the community comparator groups will not experience any intervention or treatment but are constructed to be like the treatment groups (PCPCHs) in all other study characteristics. An underlying assumption for this method is that the intervention and control groups will be the same or similar over time if there were no PCPCH policy changes (Meyer, 1995; Bertrand, Duflo, \& Mullainathan, 2001; Chunrong \& Norton, 2003). This approach to constructing PCPCH comparators is similar to propensity score matching 
(Rosenbaum \& Rubin, 1983). This matching method provides a robust approach to balancing covariates between PCPCHs, and their community comparators both improve the criteria for selection into the comparator groups and reduce confounding. The use of DD for this research provides flexibility for estimation and power to detect small treatment effects (Buckley \& Shang, 2003).

\section{Evidence Base for Empirical Methods}

DD methods have been used in health services research to measure the effects of various policy changes. Examples of DD estimation in Medical Homes research include the following: David, Gunnarsson, Saynisch, Chawla, and Nigam (2015) on the effects of MHs on ED utilization among chronic care patients; and Werner, Duggan, Duey, Zhu, \& Stuart (2013) and Friedberg, Schneider, Rosenthal, Volpp, \& Werner (2014) on the effects of PCMHs on health care utilization, quality of care, and costs. Other DD methods include the following: Wallace, McConnell, Gallia, \& Smith (2008) on investigating the effects of copayment policy change on the utilization of medical care and health care expenditures for patients in the Oregon Health Plan; Gelmon, Wallace, Sandberg, Petchel, \& Bouranis (2016) on evaluating the implementation of Oregon's PCPCH program; Currie \& Gruber (2002) on evaluating the effects of Medicaid on health care, labor supply, and the Temporary Aid to Needy Families (TANF) program. 


\section{Difference-in-Difference (D-in-D) Models}

This section explains the four main D-in-D models and their variants used for

estimating the short-term policy effects of the application of PCPCHs in Oregon. This research estimated four main different D-in-D models. The short term is defined in this research as about three years after $\mathrm{PCPCH}$ policy implementation, from October 2011 to September 2014. The D-in-D models are shown in Equations 1, 2, 3, and 4. Models 1 and 2 are utilized to answer Research Questions 1 and 2, while Model 3 will be used to answer Research Question 3. Model 2 was re-estimated based on split samples of the analytic file that stratified the aggregate average chronic disease burden measure into high and low average chronic disease burden groups. This stratification helped evaluate the effects of primary care utilization by the high and low chronic disease burden groups. Model 3 was estimated twice (Model 3a and Model 3b) on split analytic data files for high and low average chronic disease burden groups. Model 3a was used for estimating outcomes in the high chronic disease burden PCPCHs and their community comparators, while Model 3b was used for estimating outcomes in the low chronic disease burden PCPCHs and their community comparators. Models 5 and 6 were used to estimate the additive effects of the application of PCPCHs.

Model 1. The first model was developed based on the first research question:

1. What is the average chronic disease burden of PCPCHs, and how does their average chronic disease burden compare to the communities PCPCHs are in pre-post PCPCH recognition? 
Average chronic disease burden of PCPCHs and their comparator groups it $=\alpha \mathrm{PCPCH}_{i t}+\pi \operatorname{POST}_{\mathrm{t}}+\beta \mathrm{PCPCH}_{\mathrm{it}^{*}} \mathrm{POST}_{\mathrm{t}}+\mathrm{e}_{\text {it }}(1)$

In Model 1, the average chronic disease burden of PCPCHs and their comparator groups was the outcome of interest; $i$ is the aggregate observations of PCPCHs and their comparators $(1 \ldots \mathrm{n})$, where each $i$ accordingly has twice the number of practices because each $\mathrm{PCPCH}$ has a matched comparator practice; $t$ represents the time period effects, which takes on the values of 0 for pre-treatment and 1 for post-treatment periods, respectively; PCPCH represents primary care practices that were recognized by the state as PCPCHs and will take on the value 1 for PCPCHs and 0 for community comparators (non PCPCHs). Community comparators were constructed from a case-matching approach that utilized four of the predictor variables (explained above). $\alpha$ is the coefficient of the difference between PCPCHs and their community comparators over time; $\pi$ the coefficient of time (pre-post); and $\beta$ the coefficient of interaction between PCPCHs and time; $\beta$ is also the coefficient of interest because it captured the policy effects. The policy effects of the introduction of PCPCHs on chronic disease burden levels is determined by the magnitude and direction of the coefficients. The coefficients provide an estimate of the overall effects of the policy change on chronic disease burden levels in PCPCHs and their comparator groups; $e_{i t}$ represents the error term. The error term is assumed to be uncorrelated and has a mean of 0 and an unknown variance (Buckley \& Shang, 2003, Meyer, 1995).

Model 2. The second model was developed based on the second research question: 
2. How do primary care expenditures change based on the chronic disease burden of PCPCHs?

Average primary care expenditure by the chronic disease burden levels of $\mathrm{PCPCHs}_{\text {and their comparator groups }}$ it $=\alpha \mathrm{PCPCH}_{\text {it }}+\pi \mathrm{POST}_{\mathrm{t}}+\beta$

$\mathrm{PCPCH}_{\text {it }} * \mathrm{POST}_{\mathrm{t}}+\mathrm{e}_{\mathrm{it}}(2)$

In Model 2, primary care use by the average chronic disease burden of PCPCHs and their comparator groups represents the outcome of interest. This measure captures the intensity of primary care use and expenditure levels by high and low chronic disease burden patients in PCPCHs and PCPCHs and their community comparators. The outcome variable was derived from aggregating primary care expenditures per patient per $\mathrm{PCPCH}$ per quarter, then weighting the aggregate primary care expenditures by the average chronic disease burden for each group (high and low). The comparator groups were constructed from case matching; $i$ in the model represents $\mathrm{PCPCH}$ practices $(1 \ldots . . n)$, where each $i$ accordingly has twice the number of practices because each PCPCH has a matched comparator practice; $t$ is the time period effects, which takes on the values 0 for pre-treatment and 1 for post-treatment periods, respectively; $\mathrm{PCPCH}$ will take on the value 1 for PCPCHs and 0 for community comparators; $\alpha$ is the coefficient of the differences between PCPCHs and their communities over time; $\pi$ the coefficient of time (pre-post); and $\beta$ the coefficient of the interaction between PCPCHs and time. $\beta$ is the coefficient of interest because it captured the policy effects. The policy effects of the introduction of PCPCHs on the intensity of primary care use in PCPCHs was determined by the magnitude and direction of the 
coefficients, $e_{i t}$ in the model represents the error term, which is assumed to be uncorrelated and has a mean of 0 and an unknown variance (Buckley \& Shang, 2003, Meyer, 1995).

Model 3. The third model was developed based on research question 3:

3. Do PCPCHs that engage more high chronic disease burden patients have more reductions in ED and IP expenditures?

$$
\mathrm{Y}_{\text {it }}=\alpha \text { PCPCHK it }+\pi \text { POST } \mathrm{t}+\beta \text { PCPCHK it* POST } \mathrm{t}+\mathrm{e}_{\text {it }}(3)
$$

As part of this estimation, the analytic data file was split into high and low average chronic disease burden groups based on the median chronic disease burden measure. Model 3 was run twice on the two-separate chronic disease burden groups (high and low) for PCPCHs and their comparators to determine whether ED and IP utilization was different among the high and low chronic disease burden groups. $Y$ it in Model 3 represents the outcome variables of interest, namely average IP utilization and average ED utilization for the high chronic disease burden group PCPCHs and their community comparators; $i$ is the number of practices $(1 \ldots \mathrm{n})$, where each $i$ will accordingly have twice the number of practices because each PCPCH has a matched comparator practice; $t$ will represent the time period effects, which takes on the values 0 for pre-treatment and 1 for post-treatment periods, respectively. $P C P C H K$ represents IP and ED utilization by high chronic disease burden PCPCHs and their comparators; $\alpha$ is the coefficient that measures the difference in ED or IP use for high chronic disease burden PCPCHs and their comparators over time; $\pi$ will be the coefficient of time (pre-post); and $\beta$ the coefficient of the interaction between 
PCPCHK and time. $\beta$ is also the coefficient of interest because it captures the policy effects of the introduction of PCPCHs on the high chronic disease burden PCPCHs and their comparators; $e_{i t}$, on the other hand, represents the error term and like other equations, the error term is assumed to be uncorrelated and has a mean of 0 and an unknown variance (Buckley \& Shang, 2003; Meyer, 1995).

The policy effects of ED and IP utilization was evaluated by re-estimating Equation 3 to determine the utilization of ED and IP in the low chronic disease burden PCPCHs and their community comparators, then comparing the difference in ED and IP utilization among low and high chronic disease burden groups. Equation 3 was rerun as Model 3b on the low chronic disease burden PCPCHs and their comparators as specified below:

$$
\mathrm{M}_{\text {it }}=\alpha \text { PCPCHK it }+\pi \text { POST } t+\beta \text { PCPCHK it* POST } t+e_{i t}(3 b)
$$

The elements of Model $3 \mathrm{~b}$ are the same as in Model 3 above. The only difference is the outcome variable of interest denoted by $M_{i t}$, which represents average IP utilization or average ED utilization for low chronic disease burden PCPCHs and their community comparators. $M_{i t}$ is the outcome variable of interest. The overall effect was determined based on the magnitude and direction of the coefficients $\beta$ in the high and low chronic disease burden groups.

Models 4 and 5. To help determine the additive effects of the application of the PCPCH model on total ED, IP, and PC utilizations between PCPCHs and their community comparators, Models 4 and 5 were run to examine the different scenario effects. The policy effects of ED plus IP utilization were evaluated by estimating 
Equation 4 to determine the utilization of ED plus IP for the low and high chronic disease burden group PCPCHs and their community comparators, then comparing the difference in ED plus IP utilization among low and high chronic disease burden groups. Equation 4 was run twice as specified below for high and low chronic disease burden groups:

$$
\mathrm{A} \text { it }=\alpha \text { PCPCHK it }+\pi \text { POST } t+\beta \text { PCPCHK it* POST } t+e \text { it }(4)
$$

The elements of Model 4 are the same as in earlier models above. The only difference is the outcome variable of interest denoted by $A$ it, which represents average (ED plus IP) utilization for high chronic disease burden group and average (ED plus IP) utilization for low chronic disease burden PCPCHs and their community comparators. $A$ it is the outcome variable of interest. The overall effect was determined based on the magnitude and direction of the coefficients $\beta$ in the high and low chronic disease burden groups.

Model 5 was also run to examine the different scenario effects. Specifically, the policy effects of ED plus IP plus PC utilization were evaluated by estimating Equation 5 to determine the utilization of ED plus IP plus PC for the low and high chronic disease burden PCPCHs and their community comparators, then comparing the difference in ED plus IP plus PC utilization among low and high chronic disease burden groups. Equation 5 was run twice as specified below for high and low chronic disease burden PCPCHs and their comparators:

$$
\mathrm{B} \text { it }=\alpha \text { PCPCHK it }+\pi \text { POST } t+\beta \text { PCPCHK it* POST } t+e \text { it (4) }
$$


The elements of Model 5 are the same as in earlier models above. The only difference is the outcome variable of interest denoted by $B i t$, which represents average (ED plus IP plus PC) utilization for high chronic disease burden group PCPCHs and their community comparators and average (ED plus IP plus PC) utilization for low chronic disease burden PCPCHs and their community comparators. $A$ it is a binary outcome variable of interest. The overall effect was determined based on the magnitude and direction of the coefficients $\beta$ in the high and low chronic disease burden groups.

\section{Statistical Analysis}

This research used two models, a fixed effect and a generalized linear model (GLM) approach that included standard specifications for estimating the four DD models above. Data on the dependent variables were entered natively and specified to run exponential models. Log-link transformations make the dependent variables linear forms; the coefficients of the log-link transformed models become elasticities and are interpreted as measures of the rate of change in the dependent variables. Among studies that have used this approach include Wallace, McConnell, Gallia, \& Smith (2008) on the effects of copayment policy change on the utilization of medical care and health care expenditures for patients in the Oregon Health Plan. All models used the Huber-White-sandwich standard error (SE) estimation approach to help adjust for the effects of heteroskedasticity and repeated observations from before and after PCPCH recognition (Huber, 1967; White, 1980; Manning, 1998). 


\section{Limitations of Research Methods}

The main limitations include the definition of community, the match and comparison of high and low chronic disease burden PCPCHs to their control groups, and the use of the average chronic disease burden measure as a proxy measure for health risks. Community in this research was defined more narrowly than the Office of Rural Health Policy (2016) in Oregon's definition of “contiguous ZIP codes within specified primary care service areas" (p.3). This research constructed community comparators through a case-matching of ZIP codes of patients as well as age, gender, and health insurance types. This approach to creating community comparators helped match and compare high and low chronic disease burden PCPCH clinics to comparators based on the types of patients served in their respective community locations. This matching approach assumed that high and low chronic disease burden PCPCH patients will reflect their communities, but the results of the research suggest that high and low chronic disease burden PCPCHs do not currently reflect the communities in which they operate. This research compared high chronic disease burden PCPCHs to their high chronic disease burden community comparators, but the results suggest that comparing high chronic disease burden PCPCHs to their low chronic disease burden comparators could have produced more significant effects on outcomes.

The use of the All Payer All Claims (APAC) secondary data system-imposed limitations because the data contained large missing health risk scores for patients whose conditions were not yet considered high risk because their health care episodes 
had not reached chronic thresholds for generating health risk scores. A chronic disease burden was therefore developed and used as a proxy health risk measure. This chronic disease burden measure may have been limited in accuracy because it was based on 10 variable markers of chronic disease burden measures that were available in the APAC data. This measure may not have fully captured health risks because its construction did not include other elements of a comprehensive health risk measure. While other studies have used similar measures on patients' chronic conditions as proxy health risk measures, health risk scores and the number of patients' chronic conditions produce similar results, but they are different.

As measures of health risks, risk scores are more comprehensive than chronic conditions and have more predictive power because they include more variables that help comprehensively evaluate current and future health risks. This research is also a short-term study that used data on the first wave of $\mathrm{PCPCH}$ attestations from October 2011 through September 2014. PCPCH attestation data collection at the practice level has improved since the first wave of data collection. The results of this research may therefore not capture such improvements. The results may also have limited generalizability because they are exclusive to the application of Oregon's PCPCH model. 


\section{Chapter 4: Results}

The purpose of this chapter is to provide the results of the research, which are organized in tables with explanations for each table. The chapter will begin with a summary of the data analysis and regression models that were estimated to provide context for the results that follow. Preceding each table will be the associated research question, then the explanation of the results in each table. Collectively, the tables and explanations provide answers to the research questions. The tables are designed in similar format: They include similar comparative elements and are presented in the order in which they explain each research question.

\section{Summary of Data Analysis}

There are six main sets of results for the four research questions; the results include two additional tables that provide perspectives on the additive effects of the implementation of PCPCHs on the outcomes of this research. All analyses used difference-in-difference (D-in-D) methods with four main sets of dependent variables, one for each research question. For each table, the main result that will be reported is the beta (coefficient) of change, which measures the pre-post PCPCH policy effects for the control group and the pre-post $\mathrm{PCPCH}$ policy effects for the intervention group; and the D-in-D, which measures the policy effects of the changes in the treatment group based on the changes in their respective control group. The results represent practice-level effects per quarter. Therefore, the changes displayed in the result tables are interpreted as average quarterly changes in the dependent variables per patients within practice quarters. 
Two separate modified D-in-D regression models were estimated using STATA 15. Both models used random effects to account for differences in average levels of dependent variables across observations. The recognition of primary care clinics as PCPCHs began in October 2011 and is ongoing; because the recognition schedule for primary care clinics as $\mathrm{PCPCH}$ varied in this study by different start dates, quarterly time dummy variables were used to adjust for the different start dates and account for the differences across time. The results tables show these regression results as Model 1 and Model 2. The betas in Model 1 are interpreted as the magnitude of change in the dependent variable. The betas in Model 2 are interpreted as the rates of change in the dependent variables. Model 2 used a generalized linear model (GLM) approach with log transformations of the variables into linear forms. All regression models adjusted for the standard errors for repeated measures and generalized heteroskedasticity (Huber, 1967; White, 1980; Manning, 1998).

The proxy health risk measure for this research (chronic disease burden measure) was developed from aggregates of 10 chronic conditions (Ash \& Ellis, 2012; Hornbrook \& Goodman, 1996; Ash et al., 2000). Individual chronic disease burden was based on aggregates of the 10 chronic conditions described earlier in Chapters 2 and 3, while practice level chronic disease burden was measured as the average of practices' patient level chronic disease burden. The practice level chronic disease burden measure was stratified into high and low chronic disease burden groups based on the median PCPCH chronic disease burden measure and was used for the analysis in two ways: (1) stratifying PCPCH observations into high and low 
chronic disease burden groups for comparison, and (2) stratifying PCPCH

observations and their respective community comparators into high and low chronic disease burden groups based on the PCPCH chronic disease burden level, then comparing the chronic disease burden in PCPCHs (high and low) accordingly with their respective community comparator groups.

To provide additional insights into the comparative analysis of PCPCHs by high and low chronic disease burden groups, the average changes in the dependent variables for Research Questions 2 and 3 were measured from three different perspectives: comparing high and low chronic disease burden $\mathrm{PCPCHs}$, comparing low chronic disease burden PCPCHs to their respective chronic disease burden community comparators, and comparing high chronic disease burden PCPCHs to their respective chronic disease burden community comparators. The different levels of comparison helped assess whether the comparison of high versus low chronic disease burden PCPCHs did not simply reflect differences in the PCPCH communities.

\section{Results}

Research Question 1. The first research question is the following: What is the average chronic disease burden of $\mathrm{PCPCHs}$, and how does their average chronic disease burden compare to the communities $\mathrm{PCPCH}$ are in pre-post $\mathrm{PCPCH}$ recognition?

The dependent variable is chronic disease burden of PCPCHs and their community comparators, and the results are interpreted as changes in the chronic disease burden 
based on a measurement scale of $0-8$. Table 4.1 below shows the chronic disease burden of PCPCHs and their community comparators during the study period and the distribution of patient characteristics among PCPCHs and their community comparator observations. It can be inferred from Table 4.1 that the chronic disease burden of PCPCHs and their community comparators was different pre-policy implementation, about 0.89 for $\mathrm{PCPCH}$ and about 0.95 for the community comparator group. A two-tailed $t$ test was used to determine whether the difference in the chronic disease burden between PCPCHs and their communities was significantly different than zero. The result, $t(9)=3.72, p<0.05$, suggested that the difference in the chronic disease burden was statistically significant and therefore different than zero. This result suggested that the chronic disease burden of PCPCHs and their communities was significantly different pre-PCPCH policy implementation.

PCPCHs and their communities were also similar on the 10 markers of chronic disease burden measures, except for other behavioral health: 23.4 percent and 30.5 percent for PCPCHs and their communities, respectively. A two-tailed $t$ test was used to determine whether the difference in other behavioral health between PCPCHs and their communities was significantly different than zero. The result, $t(1)=39.42$, $p<0.01$, suggested that the difference in other behavioral health was statistically significant and therefore different than zero. This result suggested that the difference in other behavioral health patients in PCPCHs and their communities was significantly different. Since PCPCHs and their community comparators were not matched on the chronic disease burden, the proportional difference in other 
behavioral health therefore reflects a marker of how PCPCHs are different than their community comparators.

Table 4.1

Patient Characteristics in PCPCHs and Their Community Comparators

Table 4.1

Patient Characteristics in PCPCHs and their Community Comparators

\begin{tabular}{|c|c|c|c|c|c|c|c|}
\hline & PCPCH & Community & Sig & & РCPCH & Community & Sig \\
\hline Provider Quarters & 5,442 & 5,442 & & & & & \\
\hline Average Chronicity & 0.89 & 0.95 & $*$ & Payer Types & & & \\
\hline Gender & & & & Medicaid & $53.7 \%$ & $54.1 \%$ & \\
\hline Female & $58.3 \%$ & $58.2 \%$ & & Medicare & $10.4 \%$ & $10.3 \%$ & \\
\hline Male & $41.7 \%$ & $41.8 \%$ & & Private & $35.9 \%$ & $35.6 \%$ & \\
\hline Age Groups & & & & Chronic Conditions & & & \\
\hline $0-1$ & $3.1 \%$ & $3.1 \%$ & & COPD & $19.2 \%$ & $18.6 \%$ & \\
\hline $1-2$ & $3.5 \%$ & $3.5 \%$ & & Schizophrenia & $1.7 \%$ & $1.7 \%$ & \\
\hline $3-5$ & $7.7 \%$ & $7.7 \%$ & & Affective Disorder & $10.0 \%$ & $10.0 \%$ & \\
\hline $6-11$ & $8.0 \%$ & $8.0 \%$ & & Other Behavioral Health & $23.4 \%$ & $30.5 \%$ & $* *$ \\
\hline $12-17$ & $8.7 \%$ & $8.7 \%$ & & Diabetes & $12.6 \%$ & $12.0 \%$ & \\
\hline $18-25$ & $7.1 \%$ & $7.2 \%$ & & Chronic Heart Failure & $3.5 \%$ & $3.5 \%$ & \\
\hline $26-35$ & $10.1 \%$ & $9.9 \%$ & & Chronic Kidney Disease & $2.8 \%$ & $2.9 \%$ & \\
\hline $36-45$ & $10.8 \%$ & $10.9 \%$ & & Cardiovascular Disease & $5.4 \%$ & $5.2 \%$ & \\
\hline $46-55$ & $13.1 \%$ & $13.2 \%$ & & Coronary Heart Disease & $5.6 \%$ & $5.7 \%$ & \\
\hline $56-64$ & $13.4 \%$ & $13.5 \%$ & & Obesity & $4.8 \%$ & $4.6 \%$ & \\
\hline $64+$ & $14.5 \%$ & $14.3 \%$ & & & & & \\
\hline \multicolumn{8}{|l|}{$* \mathrm{p}<=.05$} \\
\hline$* * \mathrm{p}<=.01$ & & & & & & & \\
\hline
\end{tabular}

Table 4.2 below shows the results on the changes in the chronic disease burden post-PCPCH policy implementation. The change in chronic disease burden community comparators post measures the average change in the chronic disease burden for the community pre-post $\mathrm{PCPCH}$ policy implementation. Change in chronic disease burden $\mathrm{PCPCH}$ post measures the average change in the chronic disease burden for PCPCHs pre-post PCPCH policy implementation. The D-in-D measures the difference-in-difference of the average changes in the chronic disease 
burden for PCPCHs relative to the average change for the community comparator groups and thus provides the overall effect attributable to the $\mathrm{PCPCH}$ program.

Table 4.2

Average Changes in Chronic Disease Burden by PCPCHs

Table 4.2

Average Changes in Chronic Disease Burden by PCPCHs

\begin{tabular}{|c|c|c|c|c|c|c|c|}
\hline & \multicolumn{3}{|c|}{ Model 1} & \multicolumn{4}{|c|}{ Model 2} \\
\hline & Beta & SE & $\mathrm{P}>|\mathrm{Z}| \quad$ Sig & Beta & SE & $\mathrm{P}>|\mathrm{Z}|$ & Sig \\
\hline Change in chronic disease burden community comparators post & 0.013 & 0.01 & 0.19 & $1.41 \%$ & 0.010 & 0.177 & \\
\hline Change in chronic disease burden $\mathrm{PCPCH}$ post & 0.015 & 0.01 & 0.22 & $1.07 \%$ & 0.013 & 0.403 & \\
\hline D-in-D & 0.001 & 0.01 & 0.92 & $-0.34 \%$ & 0.015 & 0.818 & \\
\hline $\begin{array}{l}* \mathrm{p}<=.05 \\
* * \mathrm{p}<=.01\end{array}$ & & & & & & & \\
\hline
\end{tabular}

Note: Betas in Model 1 are interpreted as the magnitude of change in the dependent variable and the betas in Model 2 are interpreted as the rates of change in the dependent variable.

The betas (coefficients) of 0.013 and 0.015 in Model 1 show that the chronic disease burden increased in PCPCHs and their community comparators on the chronic disease burden scale. The results in Model 2 also suggest that the average rate of changes in the chronic disease burden in PCPCHs and their community comparators were about 1.4 percent and 1.1 percent, respectively. The D-in-D suggests that the policy effects of PCPCH implementation resulted in an increase of about 0.001 in the chronic disease burden in PCPCHs and a decrease of about 0.3 percent in the chronic disease burden in PCPCHs. However, none of the results was statistically significant and therefore indicates that the chronic disease burden in PCPCHs did not change after clinics were recognized as PCPCHs either alone or in comparison to their communities. The hypothesis that the chronic disease burden in 
PCPCHs will increase because PCPCHs will engage more chronic care patients was not supported by the results.

Research Question 2. The second research question is the following: How do primary care expenditures change based on the chronic disease burden of PCPCHs? The dependent variable for this question was average primary care expenditure per patient within a practice quarter, which was evaluated from three different perspectives. First, average changes were compared in primary care expenditures for high and low chronic disease burden PCPCHs. Second, average changes were compared in primary care expenditures for low chronic disease burden PCPCHs and their community comparators. Finally, average changes were compared in primary care expenditures for high chronic disease burden PCPCHs and their community comparators.

The stratification into high and low chronic disease burden groups helped compare PCPCHs and their community comparators based on the appropriate high and low chronic disease burden groups: high chronic disease burden PCPCHs and their communities; low chronic disease burden PCPCHs and their communities. Table 4.3 below shows the characteristics of the high and low average chronic disease burden patients. In addition to the differences in the high and low chronic disease burden groups, the characteristics of patients in Table 4.3 indicate that there were more older patients in the high chronic disease burden group, for example, 22.8 percent in the 64 and above age group, but only 7.0 percent of this age group was in the low chronic disease burden group. There was a slightly higher proportion of 
Medicaid patients in the high chronic disease burden group: 58.1 percent in the high chronic disease burden group versus 49.1 percent in the low chronic disease burden group. A higher proportion of the private payer group, 46.2 percent, was in the low chronic disease burden group, versus 26.1 percent in the high chronic disease burden group.

A two-tailed $t$ test was used to determine whether the differences in the chronic disease burden between the high and low average chronic disease burden PCPCHs was significantly different than zero. The result, $t(9)=4.46, p<0.05$, suggested that the difference in the chronic disease burden for the high and low chronic disease burden PCPCHs was statistically significant and therefore different than zero. This result indicates that the difference in the chronic disease burden for the high and low chronic disease burden PCPCHs was significantly different.

While the results on the 10 markers of chronic disease burden measures, age, gender, and payer types for the high and low chronic disease burden groups in Table 4.3 appear statistically different, independent chi square tests were conducted to determine whether the differences were statistically significant. The result on the 10 markers of chronic disease burden measures, $\chi^{2}(9)=56.92, p<0.05$, suggested that the difference in the distribution of chronic conditions was statistically significant. Therefore, the distribution of the markers of the chronic disease burden measures between high and low chronic disease burden PCPCHs is different. The result on age groups, $\chi^{2}(10)=47.22, p<0.05$, suggested that the difference in age distribution was statistically significant. Therefore, the distribution of age between high and low 
chronic disease burden PCPCHs is different. The result on gender, $\chi^{2}(1)=4.13, p<$ 0.05, suggested that the difference in the distribution of gender was statistically significant. Therefore, the distribution of gender between high and low chronic disease burden PCPCHs is different. The result on payer types, $\chi^{2}(2)=12.40, p<$ 0.05 , suggested that the difference in payer types was statistically significant.

Therefore, the distribution of payer types between high and low chronic disease burden PCPCHs is different.

Table 4.3

Patient Characteristics in High and Low Chronic Disease Burden PCPCHs

Table 4.3

Patient Characteristics in High and Low Chronic Disease Burden PCPCHs

\begin{tabular}{|c|c|c|c|c|c|c|c|}
\hline & High chronicity & Low chronicity & Sig & & High chronicity & Low chronicity & Sig \\
\hline Average chronicity & 1.18 & 0.60 & $*$ & Payer Types & & & $*$ \\
\hline Gender & & & $*$ & Medicaid & $58.1 \%$ & $49.1 \%$ & \\
\hline Female & $57.1 \%$ & $59.5 \%$ & & Medicare & $15.9 \%$ & $4.6 \%$ & \\
\hline Male & $42.9 \%$ & $40.5 \%$ & & Private & $26.1 \%$ & $46.2 \%$ & \\
\hline Age Groups & & & $*$ & Chronic Conditions & & & $*$ \\
\hline $0-1$ & $1.2 \%$ & $5.1 \%$ & & COPD & $23.7 \%$ & $15.0 \%$ & \\
\hline $1-2$ & $1.4 \%$ & $6.3 \%$ & & Schizophrenia & $2.9 \%$ & $0.6 \%$ & \\
\hline $3-5$ & $3.7 \%$ & $13.6 \%$ & & Affective Disorder & $13.2 \%$ & $6.8 \%$ & \\
\hline $6-11$ & $4.3 \%$ & $12.2 \%$ & & Other Behavioral Health & $27.1 \%$ & $19.8 \%$ & \\
\hline $12-17$ & $6.1 \%$ & $11.4 \%$ & & Diabetes & $18.3 \%$ & $7.0 \%$ & \\
\hline $18-25$ & $7.1 \%$ & $7.0 \%$ & & Chronic Heart Failure & $5.7 \%$ & $1.4 \%$ & \\
\hline $26-35$ & $10.8 \%$ & $8.9 \%$ & & Chronic Kidney Disease & $4.3 \%$ & $1.3 \%$ & \\
\hline $36-45$ & $11.9 \%$ & $9.4 \%$ & & Cardiovascular Disease & $8.3 \%$ & $2.6 \%$ & \\
\hline $46-55$ & $15.6 \%$ & $10.1 \%$ & & Coronary Heart Disease & $8.6 \%$ & $2.7 \%$ & \\
\hline $56-64$ & $15.1 \%$ & $9.0 \%$ & & Obesity & $6.1 \%$ & $3.4 \%$ & \\
\hline $64+$ & $22.8 \%$ & $7.0 \%$ & & & & & \\
\hline \multicolumn{8}{|l|}{${ }^{*} \mathrm{p}<=.05$} \\
\hline$* * \mathrm{p}<=.01$ & & & & & & & \\
\hline
\end{tabular}

Table 4.4 below shows the results on average changes in primary care expenditures per patient within a practice quarter from the three perspectives. Changes in the low and high chronic disease burden PCPCH post measures the pre- 
post average change in primary care expenditures for the low and high chronic disease burden PCPCHs, respectively. Similarly, the change in low chronic disease burden community comparators post, change in low chronic disease burden PCPCH post, change in high chronic disease burden community comparators post, and change in high chronic disease burden $\mathrm{PCPCH}$ post measure the pre-post average changes in primary care expenses for the low chronic disease burden PCPCHs and their community comparators, and the high chronic disease burden of PCPCHs and their community comparators, respectively. The D-in-D in each case measures the policy effects of PCPCH implementation on primary care expenditures from the three perspectives and shows the results as the magnitude of average changes in primary care expenditures in Model 1, and the average percentage change in primary care expenditures in Model 2. 
Table 4.4

Average Changes in Primary Care Spending by PCPCHs

Table 4.4

Average Changes in Primary Care Spending by PCPCHs

\begin{tabular}{|c|c|c|c|c|c|c|c|c|}
\hline & \multicolumn{4}{|c|}{ Model 1} & \multicolumn{4}{|c|}{ Model 2} \\
\hline & Beta & SE & $\mathrm{P}>|\mathrm{Z}|$ & Sig & Beta & SE & $\mathrm{P}>|\mathrm{Z}|$ & Sig \\
\hline \multicolumn{9}{|l|}{ High and low chronic disease burden PCPCHs } \\
\hline Change in low chronic disease burden $\mathrm{PCPCH}$ post & $-\$ 2.56$ & 3.96 & 0.52 & & $-1.9 \%$ & 0.020 & 0.341 & \\
\hline Change in high chronic disease burden $\mathrm{PCPCH}$ post & $-\$ 2.43$ & 6.64 & 0.72 & & $0.6 \%$ & 0.023 & 0.799 & \\
\hline D-in-D & $\$ 0.14$ & 7.38 & 0.99 & & $2.5 \%$ & 0.028 & 0.377 & \\
\hline \multicolumn{9}{|l|}{ Low chronic disease burden PCPCHs and Community Comparators } \\
\hline Change in low chronic disease burden community comparator post & $-\$ 4.12$ & 2.62 & 0.12 & & $-2.0 \%$ & 0.014 & 0.166 & \\
\hline Change in low chronic disease burden $\mathrm{PCPCH}$ post & $-\$ 3.33$ & 3.36 & 0.32 & & $-2.3 \%$ & 0.018 & 0.184 & \\
\hline D-in-D & $\$ 0.79$ & 4.16 & 0.85 & & $-0.4 \%$ & 0.022 & 0.872 & \\
\hline \multicolumn{9}{|l|}{ High chronic disease burden PCPCHs and Community Comparators } \\
\hline Change in high chronic disease burden community comparator post & $-\$ 0.97$ & 3.11 & 0.75 & & $0.3 \%$ & 0.017 & 0.986 & \\
\hline Change in high chronic disease burden $\mathrm{PCPCH}$ post & $-\$ 2.91$ & 6.26 & 0.64 & & $-0.7 \%$ & 0.023 & 0.763 & \\
\hline D-in-D & $-\$ 1.94$ & 6.70 & 0.77 & & $-0.7 \%$ & 0.024 & 0.764 & \\
\hline \multicolumn{9}{|l|}{$* \mathrm{p}<=.05$} \\
\hline
\end{tabular}

Note: Betas in Model 1 are interpreted as the magnitude of change (change in dollar amount) in the dependent variable, and the betas in Model 2 are interpreted as the rates of change (percentage change) in the dependent variable.

The betas (coefficients) in Model 1 for high and low chronic disease burden PCPCHs correspond with average primary care expenditures per patient within the practice quarter. The expenditures decreased by $\$ 2.56$ and $\$ 2.43$ per patient per practice quarter for the low and high chronic disease burden PCPCHs, respectively, while their D-in-D showed an increase of $\$ 0.14$ per patient per practice quarter, but the results in Table 4.4 were statistically non-significant. For the low chronic disease burden PCPCHs and their community comparators, average primary care expense decreased by $\$ 4.12$ and $\$ 3.33$ per patient per practice quarter for the low chronic 
disease burden community comparators and low chronic disease burden PCPCHs, respectively, and their D-in-D increased $\$ 0.79$ per patient per practice quarter. For the high chronic disease burden PCPCHs and their community comparators, the results suggest that the policy effects of the application of PCPCHs (D-in-D) resulted in a decrease in the average primary care expenses by $\$ 1.94$ per patient per practice quarter for high chronic disease burden PCPCHs.

The results from Model 2 suggest that average primary care expenditures decreased by about 1.9 percent for the low chronic disease burden PCPCHs and increased by about 0.6 percent for the high chronic disease burden PCPCHs. Their Din-D increased about 2.5 percent per patient per practice quarter for the low chronic disease burden PCPCHs and their community comparators. Primary care expense decreased by 2 percent and by 2.3 percent respective to high and low chronic disease burden PCPCHs and a 0.4 percent decrease in D-in-D per patient per practice quarter for the high chronic disease burden PCPCHs and their community comparators.

The policy effects (D-in-D) showed a decrease of about 0.7 percent in primary care expenditures for the high chronic disease burden PCPCHs per patient per practice quarter based on the changes in their community comparators. However, none of the results in Table 4.4 were statistically significant and therefore indicate that average primary care expense in PCPCHs did not change after clinics were recognized as PCPCHs either alone or in comparison to their communities. The results from Table 4.4 do not support the hypothesis that primary care expenses will increase for high chronic disease burden patients in $\mathrm{PCPCH}$ based on their chronic 
disease burden levels because average primary care expenditures decreased for high chronic disease burden PCPCHs in terms of both magnitude and percentage.

Research Question 3. The third research question is the following: Do PCPCHs that engage more high chronic disease burden patients have more reductions in Emergency Department (ED) and Inpatient (IP) expenditures? This question was evaluated separately for ED expenditures and for IP expenditures; the results are shown in Tables 4.5 and 4.6, respectively.

Like earlier results and tables, the results in Tables 4.5 and 4.6 are stratified into high and low chronic disease burden groups and evaluated from the three different perspectives described above. First, average changes were compared in ED and IP expenditures for high and low chronic disease burden PCPCHs. Second, average changes were compared in ED and IP expenditures for low chronic disease burden PCPCHs and their community comparators. Finally, average changes were compared in ED and IP expenditures for high chronic disease burden PCPCHs and their community comparators. The dependent variable for the results in Table 4.5 was average ED expenditure per patient within the practice quarter.

In Table 4.5 below, changes in the low and high chronic disease burden $\mathrm{PCPCH}$ post measures the pre-post average changes in primary care expenditures for the low and high chronic disease burden PCPCHs, respectively. Similarly, the change in low chronic disease burden community comparators post, change in low chronic disease burden PCPCH post, change in high chronic disease burden community comparators post, and change in high chronic disease burden PCPCH post measure 
the pre-post average changes in primary care expenses for the low chronic disease burden PCPCHs and their community comparators, and the high chronic disease burden PCPCHs and their community comparators, respectively. The D-in-D in each case measures the policy effects of PCPCH implementation on ED expenditures from the three perspectives and shows the results as the average change in the magnitude of ED expenditures in Model 1, and the average percentage change in ED expenditures in Model 2.

Table 4.5

Average Changes in Emergency Department Spending by PCPCHs

Table 4.5

Average Changes in Emergency Department Spending by PCPCHs

\begin{tabular}{|c|c|c|c|c|c|c|c|c|}
\hline & \multicolumn{4}{|c|}{ Model 1} & \multicolumn{4}{|c|}{ Model 2} \\
\hline & Beta & SE & $\mathrm{P}>|\mathrm{Z}|$ & Sig & Beta & SE & $\mathrm{P}>|\mathrm{Z}|$ & Sig \\
\hline \multicolumn{9}{|l|}{ High and low chronic disease burden PCPCHs } \\
\hline Change in low chronic disease burden PCPCH post & $-\$ 7.64$ & 3.11 & 0.01 & $* *$ & $-10.9 \%$ & 0.052 & 0.035 & * \\
\hline Change in high chronic disease burden PCPCH post & $\$ 0.36$ & 4.08 & 0.93 & & $0.9 \%$ & 0.054 & 0.862 & \\
\hline D-in-D & $\$ 8.00$ & 3.55 & 0.02 & * & $11.8 \%$ & 0.053 & 0.025 & * \\
\hline \multicolumn{9}{|l|}{ Low chronic disease burden PCPCHs and Community Comparators } \\
\hline Change in low chronic disease burden community comparator post & $\$ 0.17$ & 1.70 & 0.92 & & $1.8 \%$ & 0.034 & 0.602 & \\
\hline Change in low chronic disease burden $\mathrm{PCPCH}$ post & $\$ 4.49$ & 2.86 & 0.12 & & $-6.9 \%$ & 0.050 & 0.171 & \\
\hline D-in-D & $-\$ 4.66$ & 2.11 & 0.03 & * & $-8.7 \%$ & 0.037 & 0.020 & $*$ \\
\hline \multicolumn{9}{|l|}{ High chronic disease burden PCPCHs and Community Comparators } \\
\hline Change in high chronic disease burden community comparator post & $-\$ 1.55$ & 2.24 & 0.49 & & $1.0 \%$ & 0.028 & 0.718 & \\
\hline Change in high chronic disease burden $\mathrm{PCPCH}$ post & $-\$ 1.31$ & 4.22 & 0.76 & & $-2.3 \%$ & 0.043 & 0.598 & \\
\hline D-in-D & $\$ 0.24$ & 3.33 & 0.94 & & $-3.3 \%$ & 0.034 & 0.328 & \\
\hline \multicolumn{9}{|l|}{${ }^{*} \mathrm{p}<=.05$} \\
\hline$* * * *_{0}<=01$ & & & & & & & & \\
\hline
\end{tabular}

Note: Betas in Model 1 are interpreted as the magnitude of change (change in dollar amount) in the dependent variable and the betas in Model 2 are interpreted as the rates of change (percentage change) in the dependent variable. 
The betas (coefficients) in Model 1 for high and low chronic disease burden PCPCHs correspond with average ED expenditures per patient per practice quarter and suggest that expenditures decreased by $\$ 7.64$ and increased by $\$ 0.36$ per patient per practice quarter for the low and high chronic disease burden groups, respectively, while their D-in-D shows a statistically significant average increase of $\$ 8.00$ per patient per practice quarter. For the low chronic disease burden PCPCHs and their comparators, average ED expenses increased by $\$ 0.17$ and decreased by $\$ 4.49$ per patient per practice quarter for the low chronic disease burden community comparators and low chronic disease burden PCPCHs, respectively, and their D-in-D decreased $\$ 4.66$ per patient per practice quarter. For the high chronic disease burden PCPCHs and their community comparators, the results suggest that the policy effects of the application of PCPCHs (D-in-D) resulted in an increase in average ED expenses by $\$ 0.24$ per patient per practice quarter for high chronic disease burden PCPCHs relative to the change in the high chronic disease burden community comparators.

The results from Model 2 show that average ED expenditures decreased by about 10.9 percent for the low chronic disease burden PCPCHs and increased by about 0.9 percent for the high chronic disease burden PCPCHs per patient per practice quarter, and their D-in-D increased about 11.8 percent. For the low chronic disease burden PCPCHs and their community comparators, ED expenses increased by 1.8 percent and decreased by 6.9 percent, respectively, per patient per practice quarter and resulted in an 8.7 percent decrease in the policy effects on low chronic disease 
burden PCPCHs. For the high chronic disease burden PCPCHs and their comparators, the policy effects (D-in-D) resulted in a decrease of about 3.3 percent in average ED expenditures per patient per practice quarter relative to the change in the high chronic disease burden community comparators.

The results from Table 4.5 do not support the hypothesis that high chronic disease burden PCPCHs will achieve comparatively higher reductions in ED expenditures because average ED expenditures for high chronic disease burden PCPCHs did not change, while average ED expenditure decreases for low chronic disease burden PCPCHs were statistically significant. The D-in-D results indicated that average ED expenditure increases per patient per practice quarter were statistically significant for the high chronic disease burden PCPCHs in terms of magnitude and percentage relative to average ED expenditure decreases per patient per practice quarter for low chronic disease burden PCPCHs, which were statistically significantly while high chronic disease burden PCPCHs stayed flat.

Research Question 3b. The second iteration of the third research question is the following: Do PCPCHs that engage more high chronic disease burden patients have more reductions in Inpatient (IP) expenditures? This section of results is focused on IP expenditures by chronic disease burden levels, with results shown in Table 4.6 below. The dependent variable for the results in Table 4.6 was average IP expenditure per patient within the practice quarter. 
Table 4.6

Average Changes in Inpatient Spending by PCPCHs

Table 4.6

Average Changes in Inpatient Spending by PCPCHs

\begin{tabular}{|c|c|c|c|c|c|c|c|c|}
\hline & \multicolumn{4}{|c|}{ Model 1} & \multicolumn{4}{|c|}{ Model 2} \\
\hline & Beta & SE & $\mathrm{P}>|\mathrm{Z}|$ & Sig & Beta & SE & $\mathrm{P}>|\mathrm{Z}|$ & Sig \\
\hline \multicolumn{9}{|l|}{ High and low chronic disease burden PCPCHs } \\
\hline Change in low chronic disease burden $\mathrm{PCPCH}$ post & $-\$ 11.18$ & 16.82 & 0.51 & & $2.2 \%$ & 0.063 & 0.725 & \\
\hline Change in high chronic disease burden PCPCH post & $\$ 4.99$ & 18.49 & 0.79 & & $14.3 \%$ & 0.065 & 0.027 & * \\
\hline D-in-D & $\$ 16.17$ & 17.23 & 0.35 & & $12.1 \%$ & 0.064 & 0.050 & * \\
\hline \multicolumn{9}{|l|}{ Low chronic disease burden PCPCHs and Com munity Comparators } \\
\hline Change in low chronic disease burden community comparator post & $-\$ 1.52$ & 9.45 & 0.87 & & $-0.6 \%$ & 0.039 & 0.876 & \\
\hline Change in low chronic disease burden PCPCH post & $-\$ 6.68$ & 15.17 & 0.66 & & $-0.9 \%$ & 0.606 & 0.883 & \\
\hline D-in-D & $-\$ 5.16$ & 13.13 & 0.69 & & $-0.3 \%$ & 0.055 & 0.951 & \\
\hline \multicolumn{9}{|l|}{ High chronic disease burden PCPCHs and Com munity Comparators } \\
\hline Change in high chronic disease burden community comparator post & $-\$ 8.04$ & 12.55 & 0.52 & & $-3.2 \%$ & 0.043 & 0.448 & \\
\hline Change in high chronic disease burden PCPCH post & $\$ 0.81$ & 16.91 & 0.96 & & $2.1 \%$ & 0.058 & 0.715 & \\
\hline D-in-D & $\$ 8.85$ & 15.70 & 0.57 & & $5.4 \%$ & 0.032 & 0.050 & $*$ \\
\hline \multicolumn{9}{|l|}{$* \mathrm{p}<=.05$} \\
\hline
\end{tabular}

Note: Betas in Model 1 are interpreted as the magnitude of change (change in dollar amount) in the dependent variable, and the betas in Model 2 are interpreted as the rates of change (percentage change) in the dependent variable.

The betas (coefficients) in Model 1 for high and low chronic disease burden PCPCHs correspond to average IP expenditures per patient per practice quarter and suggest that expenditures decreased by $\$ 11.18$ and increased by $\$ 4.99$ per patient per practice quarter for the low and high chronic disease burden groups, respectively, while their D-in-D increased by $\$ 16.17$ per patient per practice quarter. For the low chronic disease burden PCPCHs and their comparators, average IP expenses decreased by $\$ 1.52$ and $\$ 6.68$ per patient per practice quarter for the low chronic disease burden community comparators and low chronic disease burden PCPCHs, 
respectively, and their D-in-D decreased $\$ 5.16$ per patient per practice quarter. For the high chronic disease burden PCPCHs and their community comparators, the results suggest that the policy effects of the application of PCPCHs (D-in-D) resulted in an increase in average IP expenses by $\$ 8.85$ per patient per practice quarter for high chronic disease burden PCPCHs compared to their community comparators.

The results from Model 2 suggest that average IP expenditures increased by about 2.2 percent for the low chronic disease burden PCPCHs and by about 14.3 percent for the high chronic disease burden PCPCHs per patient per practice quarter, with a significant D-in-D increase of about 12.1 percent. For the low chronic disease burden PCPCHs and their community comparators, IP expenses decreased by 0.6 percent and by 0.9 percent, respectively, per patient per practice quarter, with a 0.3 percent comparative decrease in the policy effects of PCPCHs on low chronic disease burden PCPCHs. For the high chronic disease burden PCPCHs and their comparators, the policy effects (D-in-D) show a significant increase of about 5.4 percent in average IP expenditures per patient per practice quarter for high chronic disease burden PCPCHs compared to their community comparators.

The results from Table 4.6 do not support the hypothesis that high chronic disease burden PCPCHs will achieve comparatively higher reductions in IP expenditures. The D-in-D results suggest that average IP expenditures per patient per practice quarter increased for the high chronic disease burden PCPCHs in magnitude and was statistically significantly in percentage terms, but comparatively, average IP expenditures per patient per practice quarter for the low chronic disease burden 
PCPCHs did not change. This result indicates that the policy effects of the implementation of PCPCHs increased average IP expenditures in proportion for the high chronic disease burden PCPCHs relative to IP expenditures for the low chronic disease burden PCPCHs that did not change.

To contextualize the policy effects of the application of PCPCHs, the models were rerun first on the sum of ED plus IP, then on the sum of ED plus IP plus PC to determine the additive effects on average changes in ED plus IP expenditures, and on average changes in ED plus IP plus PC expenditures for high chronic disease burden patients, respectively. Table 4.7 below shows the results on average ED plus IP expenditures while Table 4.8 shows the results on average ED plus IP plus PC expenditures. The dependent variable for the results in Tables 4.7 and 4.8 was the sum of average ED and IP expenditures per patient within the practice quarter, and the sum of ED and IP and PC expenditures per patient within the practice quarter, respectively. 
Table 4.7

Average Changes in Emergency Department and Inpatient Spending by PCPCHs

Table 4.7

Average Changes in Emergency Department and Inpatient Spending by PCPCHs

\begin{tabular}{|c|c|c|c|c|c|c|c|}
\hline \multicolumn{4}{|c|}{ Model 1} & \multicolumn{4}{|c|}{ Model 2} \\
\hline & Beta & SE $\quad \mathrm{P}>\mathrm{Z} \mid$ & Sig & Beta & SE & $\mathrm{P}>|\mathrm{Z}|$ & Sig \\
\hline \multicolumn{8}{|l|}{ High and low chronic disease burden PCPCHs } \\
\hline Change in low chronic disease burden PCPCH post & $-\$ 19.02$ & 18.090 .29 & & $-3.1 \%$ & 0.056 & 0.585 & \\
\hline Changc in high chronic discasc burden PCPCH post & $\$ 5.26$ & 19.130 .78 & & $8.7 \%$ & 0.055 & 0.109 & \\
\hline D-in-D & $\$ 24.28$ & 17.620 .17 & & $0.118^{*}$ & 0.055 & 0.031 & \\
\hline \multicolumn{8}{|l|}{ Low chronic disease burden PCPCHs and Community Comparators } \\
\hline Change in low chronic disease burden community comparator post & $-\$ 1.41$ & 10.180 .89 & & $-0.5 \%$ & 0.035 & 0.894 & \\
\hline Change in low chronic disease burden PCPCH post & $-\$ 11.13$ & 16.230 .49 & & $-2.5 \%$ & 0.059 & 0.669 & \\
\hline D-in-D & $-\$ 9.73$ & 13.960 .49 & & $-2.1 \%$ & 0.049 & 0.675 & \\
\hline \multicolumn{8}{|l|}{ High chronic disease burden PCPCHs and Community Comparators } \\
\hline Change in high chronic disease burden com munity comparator post & $-\$ 9.29$ & 13.220 .48 & & $-3.1 \%$ & 0.038 & 0.407 & \\
\hline Change in high chronic disease burden $\mathrm{PCPCH}$ post & $\$ 0.50$ & 17.240 .90 & & $0.5 \%$ & 0.051 & 0.916 & \\
\hline D-in-D & $\$ 9.34$ & 15.660 .55 & & $3.7 \%$ & 0.043 & 0.398 & \\
\hline \multicolumn{8}{|l|}{$* \mathrm{p}<=.05$} \\
\hline$* * \mathrm{p}<=.01$ & & & & & & & \\
\hline
\end{tabular}

Note: Betas in Model 1 are interpreted as the magnitude of change (change in dollar amount) in the dependent variable, and the betas in Model 2 are interpreted as the rates of change (percentage change) in the dependent variable.

The results from Table 4.7 confirm the earlier results above on average ED and IP expenditures by different chronic disease burden levels. The beta (coefficients) on the policy effects of the application of PCPCHs (D-in-D) for high chronic disease burden PCPCHs suggests that average ED plus IP expenditures increased in magnitude and percentage for the high chronic disease burden PCPCHs by $\$ 24.28$ and 11.8 percent, respectively, but decreased for the low chronic disease burden PCPCHs by $\$ 9.73$ and 2.1 percent per patient per practice quarter, respectively. For high chronic disease burden PCPCHs, the D-in-D results suggest that average ED 
plus IP expenditures increased by about $\$ 9.34$ and by 3.75 percent per patient per practice quarter, respectively, for high chronic disease burden PCPCHs compared to their high chronic disease burden community comparators. However, only the proportional change in D-in-D for the high chronic disease burden PCPCHs was statistically significant. This result indicates that the policy effects of the implementation of PCPCHs increased average ED plus IP expenditures in proportion for the high chronic disease burden PCPCHs relative to ED plus IP expenditures for the low chronic disease burden PCPCHs that did not change.

The results from Table 4.8 below also confirm earlier results above on average ED, IP, and PC expenditures by different chronic disease burden levels. The beta (coefficients) on the policy effects of the application of PCPCHs (D-in-D) for high chronic disease burden PCPCHs suggests that average ED plus IP plus PC expenditures increased in magnitude and percentage terms for the high chronic disease burden PCPCHs by $\$ 23.23$ and 6.1 percent per patient per practice quarter, respectively, but decreased for the low chronic disease burden PCPCHs by $\$ 8.89$ and 1.6 percent per patient per practice quarter, respectively. For the high chronic disease burden PCPCHs, the D-in-D results suggest that average ED plus IP plus PC expenditures increased by about $\$ 6.76$ and by 1.2 percent per patient per practice quarter, respectively, for high chronic disease burden PCPCHs compared to their high chronic disease burden community comparators. However, none of the results were statistically significant and therefore indicated that average ED plus IP plus PC 
expenses did not change after clinics were recognized as PCPCHs either alone or in comparison to their communities.

\section{Table 4.8}

Average Changes in Emergency Department, Inpatient, and Primary Care Spending by PCPCHs

Table 4.8

Average Changes in Emergency Department, Inpatient and Primary Care Spending by PCPCHs

\begin{tabular}{|c|c|c|c|c|c|c|c|}
\hline & \multicolumn{4}{|c|}{ Model 1} & \multicolumn{3}{|c|}{ Model 2} \\
\hline & Beta & $\mathrm{SE}$ & $\mathrm{P}>|\mathrm{Z}|$ & Sig & Beta & SE & $\mathrm{P}>|\mathrm{Z}|$ \\
\hline \multicolumn{8}{|l|}{ High and low chronic disease burden РСРСНs } \\
\hline Change in low chronic disease burden $\mathrm{PCPCH}$ post & $-\$ 21.98$ & 19.19 & 0.25 & & $-2.3 \%$ & 0.333 & 0.496 \\
\hline Change in high chronic disease burden $\mathrm{PCPCH}$ post & $\$ 1.25$ & 20.55 & 0.95 & & $3.8 \%$ & 0.035 & 0.276 \\
\hline D-in-D & $\$ 23.23$ & 20.15 & 0.25 & & $6.1 \%$ & 0.037 & 0.102 \\
\hline \multicolumn{8}{|l|}{ Low chronic disease burden PCPCHs and Community Comparators } \\
\hline Change in low chronic disease burden community comparator post & $-\$ 5.56$ & 11.22 & 0.62 & & $-1.2 \%$ & 0.024 & 0.621 \\
\hline Change in low chronic disease burden $\mathrm{PCPCH}$ post & $-\$ 14.45$ & 17.08 & 0.40 & & $-2.7 \%$ & 0.037 & 0.460 \\
\hline D-in-D & $-\$ 8.89$ & 15.40 & 0.56 & & $-1.6 \%$ & 0.032 & 0.629 \\
\hline \multicolumn{8}{|l|}{ High chronic disease burden PCPCHs and Community Comparators } \\
\hline Change in high chronic disease burden community comparator post & $-\$ 10.64$ & 14.32 & 0.46 & & $-1.7 \%$ & 0.027 & 0.540 \\
\hline Change in high chronic disease burden $\mathrm{PCPCH}$ post & $-\$ 3.88$ & 18.58 & 0.83 & & $-0.4 \%$ & 0.035 & 0.901 \\
\hline D-in-D & $\$ 6.76$ & 17.58 & 0.70 & & $1.2 \%$ & 0.032 & 0.704 \\
\hline
\end{tabular}

Note: Betas in Model 1 are interpreted as the magnitude of change (change in dollar amount) in the dependent variable, and the betas in Model 2 are interpreted as the rates of change (percentage change) in the dependent variable.

\section{Summary of Results}

The results of the research suggest that the hypotheses that the recognition of primary care clinics as PCPCHs will increase chronic disease burden levels in PCPCHs because the model's approach to care will benefit high chronic disease burden patients, increase primary care expenditures for high chronic disease burden PCPCHs, and reduce ED and IP utilizations and expenditures for high chronic disease 
burden PCPCHs were not supported by the results, which showed that chronic disease burden levels in PCPCHs did not change after primary care clinics were recognized as PCPCHs. In terms of average primary care expenditure for high chronic disease burden PCPCHs, the policy effects (D-in-D) showed some changes in average primary care expenditures per patient per practice quarter; however, none of the changes were statistically significant. These results indicated that average primary care expenditures in PCPCHs did not change after clinics were recognized as PCPCHs either alone or in comparison to their communities. The results on average primary care expenditures did not support the hypothesis that primary care expenses will increase for high chronic disease burden patients in PCPCH based on their chronic disease burden levels.

For average ED expenditures for high chronic disease burden PCPCHs, the Din-D results indicated that the average increases in ED expenditures per patient per practice quarter were statistically significant for the high chronic disease burden PCPCHs in terms of magnitude and percentage relative to average decreases in ED expenditures for low chronic disease burden PCPCHs, which were statistically significant, while average ED expenditures for high chronic disease burden PCPCHs stayed flat. The results do not support the hypothesis that high chronic disease burden PCPCHs will achieve comparatively higher reductions in ED expenditures.

Similarly, the D-in-D results for IP expenditures for high chronic disease burden PCPCHs suggest that average IP expenditures increased for high chronic disease burden PCPCHs in magnitude and were statistically significant in percentage 
terms per patient per practice quarter, but comparatively, average IP expenditures per patient per practice quarter for the low chronic disease burden PCPCHs did not change. The results, therefore, do not support the hypothesis that high chronic disease burden PCPCHs will achieve comparatively higher reductions in IP expenditures. The results on the additive effects of ED plus IP and ED plus IP plus PC did not show statistically significant changes, which indicated that average ED plus IP and average ED plus IP plus PC expenses per patient per practice quarter did not change after clinics were recognized as PCPCHs either alone or in comparison to their communities. 


\section{Chapter 5: Discussion}

The objective of this research was a relative effectiveness evaluation of the application of the PCPCH model and approach to primary care in Oregon based on the chronic disease burden of PCPCHs. PCPCHs were introduced in Oregon as part of a state-wide health reform initiative that was ushered in by the passage of HB 2009 to improve upstream care through increased access to comprehensive primary care services and reduce downstream demand for Emergency Department (ED) and inpatient (IP) utilization and their associated health care expenditures. Based on the expectation that the model can help reduce ED and IP expenditures and overall health care costs through improved comprehensive primary care, especially for chronic care patients, and based on the mixed evidence on reductions in ED and IP expenditures (Jackson \& Williams, 2015; Pikes et al., 2011; Friedberg et al., 2014; Baseman, Boccuti, Moon, Griffin, \& Dutta, 2016), this research was designed to evaluate the application of $\mathrm{PCPCHs}$ with a focus on $\mathrm{PCPCH}$ practices that treat greater proportions of chronic care patients. A step-wise approach was used to help answer three questions:

1. What is the average chronic disease burden of PCPCHs, and how does their average chronic disease burden compare to the communities PCPCHs are in pre-post PCPCH recognition?

2. How do primary care expenditures change based on the chronic disease burden of PCPCHs? 
3. Do PCPCHs that engage more high chronic disease burden patients have more reductions in ED and IP expenditures?

A natural experimental design that utilized difference-in-difference methods was used to measure outcome differences pre-post PCPCH policy implementation and compare the outcome differences between PCPCHs and their community comparators. The theoretical perspectives that informed this research were Risk Selection and Complex Adaptive Systems (CAS). The theory lens postulates that rational economic agents, including health care delivery systems, that operate in competitive delivery system environments will adapt to information and other signals from their environments for competitive advantage and organizational survival. The theory lens suggests that chronic disease burden levels in clinics that become PCPCHs will change in response to the policy, program implementation, and influence changes in short-term outcomes as a result.

This research postulated that the chronic disease burden of PCPCHs will increase and result in reductions in ED and IP expenditures in the short term because chronic care patients will receive improved comprehensive primary care services, which will translate into reductions in the overuse and misuse of ED and IP services. The remaining sections of this chapter will be organized as follows: discussions of the results: chronic disease burden levels in $\mathrm{PCPCHs}$, primary care expenditures in PCPCHs, emergency department and inpatient expenditures in PCPCHs; conclusions of the research; limitations of the research; implications for policy development; recommendations for future research. 


\section{Chronic Disease Burden Levels in PCPCHs}

This research hypothesized that PCPCHs will initially have chronic disease burden levels that are equal to their community comparators, but the chronic disease burden in PCPCHs will be higher after clinics' recognition as PCPCHs. This hypothesis was based on the expectation that the model's emphasis on comprehensive primary care through improved care coordination, integration, and continuity is designed to engage and improve care for patients who have chronic physical and behavioral health conditions. Primary care clinics that become PCPCHs may be inclined to engage more chronic care patients and provide such patients with comprehensive primary care services that will reduce their dependence on ED and IP for primary care services and translate into reductions in ED and IP expenditures.

The results suggest that the chronic disease burden in PCPCHs was significantly different than their community pre-policy implementation, but the chronic disease burden in PCPCHs did not change after PCPCH recognition. Compared to their communities, the chronic disease burden in PCPCHs decreased after clinics were recognized as PCPCHs by about 0.3 percent, but this change was not statistically significant; therefore, the null hypothesis that there was no change was not rejected. The results suggest that primary care clinics that became PCPCHs did not change the patients they served either alone or with their communities. The expectation was that PCPCHs would engage more chronic care patients after recognition. 
Among the likely explanations for this result include the availability of information for patients to evaluate and determine the benefits of PCPCHs for their care, and the way patients can identify and switch to PCPCHs for their care. The results also suggest that some $\mathrm{PCPCHs}$ may not have the capacity to engage more chronic care patients because their environments are fixed. Such clinics may be operating in communities that predetermine the patient populations from which they draw. For example, PCPCH practice specialization could predetermine the types of patients they engage and the chronic disease burden levels of such patients. There could be other fixed practice level characteristics that help explain why the chronic disease burden in PCPCHs are not changing.

While the results suggest that the chronic disease burden in PCPCHs and their communities were similar on most of the markers of chronic disease burden measures (Table 4.1), they were significantly different on other behavioral health conditions. This statistically significant difference appears to be the main driver of the difference between PCPCHs and their communities because they were relatively similar on all the other markers of chronic disease burden measures. The size of the difference in other behavioral health conditions is important because PCPCHs are designed to continuously coordinate and integrate chronic physical and behavioral health care as part of providing comprehensive primary care services for their patients. Insights into who these patients are and why they are not engaged in PCPCHs will be important because the expectation was that PCPCHs would engage such patients. 
Since PCPCHs are not changing the patients they serve, some of the other behavioral health condition patients who need PCPCH care but are currently not engaged or receiving PCPCH care may have gaps in their receipt of appropriate care. This result implies that in some respects, access to and the use of primary care services may be uneven in unexpected ways for other behavioral health condition patients. The results on the non-changing chronic disease burden levels in PCPCHs imply that PCPCHs' capacity to address such care gaps, i.e. coordinating and integrating physical and behavioral health care for other behavioral health conditions patients, may be currently limited. PCPCH practices may therefore need some help to address such gaps.

The theory of risk selection provides some insights on the results for chronic disease burden levels in PCPCHs. This theory lens posits that health risks are heterogeneous, not prized, and unevenly distributed in populations, and due to the transactions cost associated with separating and pricing health risks, most reimbursements are based on the average expected cost approach. Since health risks are not prized, the expectation may not be realistic that PCPCHs will engage high chronic disease burden patients without well-aligned and explicit incentives, reimbursements, or accountability metrics to encourage $\mathrm{PCPCHs}$ to engage a higher case mix of chronic disease burden patients. While various alternative payments were implemented to incentivize PCPCHs to improve care for all patients, most of the payment incentives were not specifically aligned to incentivize PCPCHs to engage a higher case mix of chronic care patients. Without well-aligned incentives to 
encourage PCPCHs to engage a higher case mix of chronic care patients, PCPCHs may choose to focus on engaging a low average case mix of chronic disease burden patients. However, the results of the research do not suggest evidence on active risk selection.

The heterogeneity of health risks and the uneven distribution of health risks suggest that health risks may not be evenly distributed across communities, but they may be evenly distributed within communities. The chronic disease burden in PCPCHs, therefore, suggests that PCPCHs are largely like their communities, and the distribution of the chronic disease burden is similar among primary care providers within the communities in which PCPCHs are located. The results on chronic disease burden, i.e. PCPCHs are not changing the chronic disease burden mix of the patients they are engaging, could be explained by the presence of information asymmetry in the health care delivery system environment that affects the information patients have and need to make informed choices about PCPCH providers. Patients would choose PCPCHs for their care if they have information about the benefits of PCPCH care. This statement also presumes that high chronic disease burden patients would choose PCPCHs, but this is not clear. Providing more information about PCPCHs could be beneficial for patients and helpful for changing the chronic disease burden mix of PCPCHs in both the short and medium terms.

\section{Primary Care Expenditures in PCPCHs}

At the core of the Medical Home (MH) model is the delivery of comprehensive primary care upstream to create downstream delivery system benefits. 
The model's emphasis on primary care was to demonstrate the value of primary care, especially for chronic care patients. Based on the expected benefits of primary care delivery in PCPCHs, this research tested the hypothesis that average primary care expenditures will be higher for high average chronic disease burden PCPCHs based on the expectation that PCPCHs will provide more comprehensive primary care services to higher average chronic disease burden patients.

The results on average primary care expenditures suggest that primary care expenditures decreased per patient per practice quarter for low and high chronic disease burden PCPCHs. However, these changes were not statistically significant; therefore, the null hypothesis of no changes in primary care expenditures was not rejected. This conclusion implies that there was no significant difference in primary care expenditures for high and low chronic disease burden PCPCHs. The hypothesis that average primary care expenditures will increase for high chronic disease burden PCPCHs because they will have more high chronic disease burden patients and provide such patients with more comprehensive primary care services relative to low chronic disease burden patients was not supported by the results. The results on primary care expenditures could imply that the levels of primary care services in PCPCHs are already high, and patients are receiving appropriate primary care services. Therefore, average primary care services or their associated expenditures did not change significantly.

Among the conjectures from the results on average primary care expenditures in PCPCHs are practice level adaptive behaviors that ensured the delivery of effective 
and efficient primary care services based on patients' health care needs. As a result, the volume of primary care services may not have changed, but the quality and value of primary care services may have changed in other important ways that were not the focus of this research and therefore not measured. For example, Ralston et al. (2009) and Reid et al. (2009) reported in their evaluations of Medical Homes in Group Health Cooperatives that in-person primary care utilization decreased while overall primary care access increased through secure phone and email communications. Health care utilization decreased by 29 percent as a result, but the quality of care remained the same.

\section{Emergency Department and Inpatient Expenditures in PCPCHs}

The construct of improving primary care services upstream to reduce downstream demand for ED and IP has been used consistently to describe the contributions of Medical Homes to delivery systems improvement. While this relationship appears intuitive, the research evidence is mixed on improvements in ED and IP utilization, especially for chronic care patients. Based on this knowledge, this research tested the hypothesis that $\mathrm{PCPCHs}$ will achieve higher average reductions in ED and IP expenditures after recognition based on the expectation that patients with higher average chronic disease burden will have greater response to upstream primary care services that translate into decreases in average ED and IP expenditures downstream.

The results on average ED expenditures suggest that average expenditure increases per patient per practice quarter for high chronic disease burden PCPCHs 
were statistically significant in magnitude and percentage relative to average ED expenditure decreases per patient per practice quarter for low chronic disease burden PCPCHs that were statistically significant, while expenditures for high chronic disease burden PCPCH patients remained flat. The results do not support the hypothesis that ED expenditures will decrease for high chronic disease burden PCPCHs. The results could imply that high chronic disease burden patients may have less overuse and misuse of ED expenditures to relinquish in the short term because of their health conditions and need for such services. Low chronic disease burden patients, on the other hand, may have more overuse and misuse of ED and IP to relinquish as reflected in the average changes in expenditures by the different chronic disease burden groups.

The results suggesting that the largest decreases in average ED expenditures per patient per practice quarter were from low chronic disease burden PCPCHs imply that focusing on low chronic disease burden patients in PCPCHs may help reduce the inappropriate utilization of ED services and their associated expenditures. Similarly, the results on IP expenditures suggest that average IP expenditures increased significantly in rate of change for high chronic disease burden PCPCHs compared to low chronic disease burden PCPCHs. The results on ED and IP expenditures do not support the hypothesis that high chronic disease burden PCPCHs will achieve higher average reductions in ED and IP expenditures than before $\mathrm{PCPCH}$ recognition.

The results on the additive effects of the changes in average ED, IP, and PC for high chronic disease burden PCPCHs reflect the results on their individual effects. 
Other research confirms the mixed results for ED and IP utilization for high chronic disease burden patients (Dorr, Wilcox, Brunker, Burdon, \& Donnelly, 2008; Jackson et al., 2013; Friedberg et al., 2014). Based on the results from comparing high and low chronic disease burden PCPCHs, it can be argued that the expectations that high chronic disease burden PCPCHs will reduce ED and IP expenditures and exert downward pressure on health care costs were not supported. The results do suggest that the distribution of chronic disease burden in PCPCHs is important for the evaluation of ED and IP expenditures in the short term, but in the opposite direction than expected. Focusing on low and medium chronic disease burden patients may help reduce $\mathrm{ED}$ and IP expenditures in the short and medium terms.

The relatively different results on average ED and IP expenditure reductions for high versus low average chronic disease burden PCPCHs confirm generally the mixed evidence in the literature on ED and IP expenditure reductions, and specifically from high chronic disease burden patients. The results also suggest that the interrelationships between the health conditions of patients in PCPCHs, the care such patients receive in PCPCHs, and how their care translates into ED and IP utilization and expenditures may be more complex than initially conceived by this research. The expectation by proponents of the model that providing improved upstream care could translate into reductions in ED and IP utilization and expenditures downstream may be ambitious and need refinement and further insights based on the evolving evidence from the model's applications. 
The results on ED and IP expenditures could be explained from the perspective of CAS, which suggests that health care delivery in PCPCHs may have complex equilibria based on the different health conditions of patients and interactions in their environments (Holden, 2005). Because patients are heterogeneous in their health conditions, they will likely have different health care service needs and interact differently with existing and new health care delivery structures for their service needs. The outcomes of patients' interactions with the different health service structures may be different as well. The complexity of patients' health needs, the choices they make to pursue health care, and the interactions in the health system environment that impact patients and the outcomes of their care may not be fully understood.

Our expectations of the process and outcomes of PCPCH care, especially for chronic care patients, may need to evolve based on the emerging evidence. For example, the expectation that PCPCHs could help reduce ED and IP expenditures assumed that the overuse and misuse of ED and IP utilization would be higher for high chronic disease burden patients; therefore, engaging high chronic disease burden patients in PCPCHs could help reduce such inappropriate utilization. But the results suggest the opposite. The overuse and misuse of ED and IP utilization may be higher for low chronic disease burden patients. Engaging low chronic disease burden patients in PCPCHs, therefore, may help reduce average ED and IP expenditures because low chronic disease burden patients may have more inappropriate use to relinquish. High chronic disease burden patients, on the other hand, may have higher 
ED and IP use because they need such care, which can be considered appropriate (versus inappropriate) use of ED and IP services.

The results on average ED and IP expenditures per patient per practice quarter for high chronic disease burden PCPCHs also suggest that expenditures are not decreasing at the same proportion as low chronic disease burden PCPCHs, but they are not increasing at a higher proportion either (expenditures were unchanged). Based on the expectation that high chronic disease burden patients will need and use ED and IP services because of their health status, the emphasis may need to be on the efficient and effective utilization of ED, IP, and other non-ED and IP health care services by high chronic disease burden patients in PCPCHs. From this perspective, it can be argued that the results suggesting that average ED and IP expenditures for high chronic disease burden PCPCHs did not change, should be expected, because PCPCH patients may have effectively and efficiently used ED and IP services.

This research stratified PCPCHs into high and low chronic disease burden groups based on the chronic disease burden levels of their patients. The different results on average ED and IP expenditures suggest that there may be some inherent differences with the comparison of PCPCHs based on the high and low chronic disease burden groupings, or some misunderstanding of the complexity of the care transformations for high and low chronic disease burden patients or high and low chronic disease burden PCPCHs. Reviewing the appropriate comparators to use for the high and low groups may help capture the unobserved or poorly understood differences in the high and low chronic disease burden patient populations or the high 
and low chronic disease burden PCPCHs. Furthermore, the development and use of the chronic disease burden as a proxy health risk measure for this research assumed that high average chronic disease burden patients are homogenous. However, the results suggest that high average chronic disease burden patients in PCPCHs may be different. The theory of CAS suggests that high average chronic disease burden patients in PCPCHs may interact or be associated with different and complex environmental factors in different ways that lead to complex outcome paths.

The results in Tables 4.1 and 4.3 on the characteristics of patients in PCPCHs and high chronic disease burden patients suggest that these patients represent a larger proportion of Medicaid enrollees and a larger variation of age groups. This discovery suggests that there could be some unobserved and unmeasured differences in the social determinants of health for these patients that may be impacting the results on average ED and IP expenditures. Also, figure 3.1 shows the scope of social determinates of health including economic, neighborhood, environment, and education, their interactions, and their potential impacts on access to health care and potentially, the use of ED and IP especially for Medicaid patients. Differences in social determinants of health such as health literacy, healthy foods, poverty, access to shelter, and the availability and reliability of transportation have been found to impact continuous access to healthcare services including medication adherence, and health outcomes. Starfield, Shi \& Macinko (2005), Goodman et al. (2003), and Cowling et al. (2013) suggested that differences in social determinants of health have impacts on ED and IP utilization and expenditures. Further insights into the unobserved and 
unmeasured differences in the social determinants of health for high chronic disease burden patients in PCPCHs could help explain their utilization of ED, IP, and other services.

\section{Conclusions}

Based on the expectation that PCPCHs' approach to care would engage more high chronic disease burden patients and improve the outcomes of care for such patients, this research tested the hypotheses that the relative effectiveness of PCPCHs to reduce ED and IP expenditures would depend on engaging high chronic disease burden patients, providing such patients with increased PC services upstream that translate into decreases in average ED and IP expenditures downstream. The results of the research did not support the hypotheses but suggested that the distribution of chronic disease burden in PCPCHs is important for evaluating the relative effectiveness of the application of the model, but in the opposite direction than expected. The distribution of the chronic disease burden in PCPCHs impacted the magnitude and rate of decreases in average ED and IP expenditures in the short term. This insight is important for policy-related decisions in PCPCHs and for future research because low chronic disease burden patients in PCPCHs achieved more ED and IP savings compared to high chronic disease burden patients in PCPCHs, whose expenditures stayed flat.

The results suggest that the chronic disease burden in PCPCHs was unchanged after primary care clinics' recognition as PCPCHs even though it was assumed that the chronic disease burden would increase. This suggests that PCPCHs 
are not changing the types of patients they serve, even if they are changing the care they provide to their patients. Table 4.1 on the characteristics of PCPCH patients suggests that there are statistically significant differences in the proportions of other behavioral health conditions patients in PCPCHs and the community: 23.4 percent versus 30.5 percent, respectively. This result is important because part of the organization of care in PCPCHs is based on the effective coordination and integration of care across the care continuum for physical and behavioral health conditions patients. The difference in the proportion of other behavioral health patients in PCPCHs suggests that some of these patients who may need and can benefit from the PCPCH approach to care are not engaged in PCPCHs. Further research into who these patients are, why they are not engaged in PCPCHs, and how they can be engaged will provide important insights into the application of the model.

Average PC expenditures per patient per practice quarter for high chronic disease burden PCPCHs relative to low chronic disease burden PCPCHs did not change after policy implementation, but the hypothesis was that PC expenditures for high chronic disease burden PCPCHs would increase because clinics would engage more high chronic disease burden patients who need and use more PC services. One conjecture from this result is that both high and low chronic disease burden PCPCHs provided effective and efficient PC services, and PC services may have changed in other ways that were not the focus of this research and therefore not measured. Average ED and IP expenditures per patient per practice quarter for high chronic disease burden PCPCHs did not change in terms of magnitude or percentage 
relative to average ED and IP expenditures for low chronic disease burden PCPCHs, but average expenditures per patient per practice quarter for low chronic disease burden PCPCHs decreased while average expenditures for high chronic disease burden PCPCHs stayed flat. It can be inferred from these results that average ED and IP expenditures per patient per practice quarter for high chronic disease burden PCPCHs did not decrease as expected compared to low chronic disease burden PCPCHs. Therefore, focusing on low chronic disease burden PCPCHs could help achieve the expected reductions in average ED and IP expenditures in the short term. These results imply that chronic disease burden levels in PCPCHs are important for evaluating the relative effectiveness of the model's application because the distribution of the chronic disease burden in PCPCHs impacted average ED and IP utilization and savings. Stratifying PCPCHs into high average and low average chronic disease burden PCPCHs provided important insights into ED and IP expenditures by high average and low average chronic disease burden groups.

Furthermore, the results suggest that current knowledge on the performance of PCPCHs may not capture the dynamic interactions of the model's application. The different results on average ED and IP expenditures by high and low average chronic disease burden PCPCHs suggest that the interrelationships among the health status of patients in PCPCHs and specific PCPCH effects, such as the type of care patients receive and how such care translates into decreases in ED and IP utilization and expenditures, may be more complex than initially envisioned by this research. The expectation by proponents of the model that providing improved upstream care would 
translate into reductions in ED and IP utilization and expenditures downstream may need further refinement based on the evolving evidence from the model's application in different delivery systems.

The approach to constructing the chronic disease burden measure and stratifying the chronic disease burden measure into high average and low average chronic disease burden PCPCHs assumed that patients are homogenous within chronic disease burden levels; therefore, high or low chronic disease burden patients in high average chronic disease burden PCPCHs resemble high or low chronic disease burden patients in low average chronic disease burden PCPCHs. However, the relatively different results on the average ED and IP expenditures per patient per practice quarter for high average chronic disease burden PCPCHs suggest that there may be some unobserved differences in the health system environment of these PCPCHs that interact with patient chronic disease burden, or with the patients themselves, and distinguishes them from patients in other PCPCHs.

The proportion of Medicaid patients in the high chronic disease burden PCPCH group suggests that the differences may be related to higher social determinants of health and related health systems deficiencies (displayed and explained in figure 3.1) that impact average ED and IP utilization and expenditures for patients in high average chronic disease burden PCPCHs. The use of the chronic disease burden measure and the approach to matching and comparing high and low chronic disease burden PCPCHs in this research did not envision or measure the 
different effects of the dynamic interactions in the environments of high and low chronic disease burden PCPCHs.

It can be inferred from the results of this research that the complex interactions in the health care delivery system environment in which PCPCHs operate and the effects of such complexity on expected outcomes may not have been anticipated by proponents of the model or adjusted for in their expectations. The differences in outcomes, the actual and expected, could be attributed to the initial expectations about the model's application. Crabtree et al. (2011) observed from historical evidence on primary care transformation that improving outcomes of care is complicated and may only be achieved from "major changes to the interdependent relationships among agents" (p. S30). This insight suggests that PCPCHs may not be able to engage all high chronic disease burden patients in ways that result in significant and sustainable decreases in average ED and IP utilization and expenditures because some high average chronic disease burden patients live in different or difficult social environments that have limited health system support beyond PCPCHs; such patients may therefore need and use ED and IP services accordingly.

\section{Limitations of the Research}

Based on the design of this research, the sources of data, and the results, several limitations emerged. The main limitations include the definition of community, the use of PCPCHs as the unit of analysis, matching and comparing high and low chronic disease burden PCPCHs to their control groups, and the use of the 
chronic disease burden measure as a proxy measure for health risks. This research evaluated the chronic disease burden of PCPCHs and their effects on specific outcomes; the results, therefore, constitute practice-level instead of individual-level effects. The individual results are also different from an overall evaluation of the PCPCH program. Since PCPCHs may have different patient population sizes across different practices, the average effects (changes) may be very different among practices than on individual patients. The use of PCPCHs as the unit of analysis for this research was important for determining the policy effects of the application of the model. The results on the average changes in PCPCHs are therefore different from the results that would be obtained from an evaluation of the overall effects of the PCPCH program, which would have been based on weighting the number of individuals who are receiving primary care services in PCPCHs.

Primary care communities in this research were defined more narrowly than the Office of Rural Health Policy (2016) in Oregon's definition of primary care service areas, which are "contiguous ZIP codes within specified primary care service areas" (p. 3). This research constructed community comparators through case matching patients' ZIP codes, age, gender, and health insurance types. This approach to creating community comparators helped match and compare high and low average chronic disease burden PCPCH clinics to comparators based on the types of patients served in their respective community locations. This matching approach assumed that patients are homogenous within high average and low average chronic disease burden practices; therefore, high and low chronic disease burden patients in PCPCHs would 
reflect the PCPCHs' communities. The results, however, suggest that high and low chronic disease burden PCPCHs do not currently reflect the communities in which they operate.

This research compared the chronic disease burden on three levels: (1) high average chronic disease burden PCPCHs to low average chronic disease burden PCPCHs, (2) low average chronic disease burden PCPCHs to their low average community comparators, and (3) high average chronic disease burden PCPCHs to their high average community comparators. However, the results of the research suggest that this approach to comparing PCPCHs (high average chronic disease burden PCPCHs to low average chronic disease burden PCPCHs) may have affected the outcomes achieved. Additionally, the use of the All Payer All Claims (APAC) secondary data system-imposed limitations because the data omitted many health risk scores for patients whose conditions were not yet considered high risk because their health care episodes had not reached chronic thresholds for generating health risk scores.

A chronic disease burden measure was therefore developed and used as a proxy health risk measure for this research. This chronic disease burden measure was limited because it was based on 10 variable markers of chronic conditions that were available in the APAC data. Other studies have used similar measures on patients' chronic conditions as proxy health risk measures (Ash et al., 2000; Hornbrook \& Goodman, 1996), but while health risk scores and the number of patients' chronic conditions are similar, they produced relatively different results in several ways. As 
measures of health risks, risk scores are more comprehensive than chronic conditions; they have more predictive power because they include more variables that help comprehensively evaluate current and future health risks.

This research is also a short-term study that focused on measuring the effects of high and low average chronic disease burden in PCPCHs on a limited set of outcome variables that are used by policymakers in policy decision-making. The results were based on data on the first wave of $\mathrm{PCPCH}$ attestation from October 2011 through September 2014, but PCPCH attestation data collection at the practice level has improved since the first wave of data collection. The model has also been implemented more broadly across the state over time. The results of this research may not capture such improvements and may also have limited generalizability because they are exclusive to the application of Oregon's PCPCH model.

\section{Implications for Policy Development}

This research evaluated the relative effectiveness of the application of PCPCHs with a focus on high chronic disease burden patients to determine whether engaging high chronic disease burden patients in PCPCHs decreased average ED and IP expenditures. The results of this research have some implications for PCPCH policy development, specifically for broad policy development on the applications of the model.

The results suggest that focusing on chronic conditions of patients in PCPCHs is important for evaluating the relative effectiveness of the model's application because decreases in average ED and IP expenditures per patient per practice quarter 
were impacted by the distribution of the chronic disease burden in PCPCHs.

Decreases in average ED and IP expenditures per patient per practice quarter were comparatively greater for low average chronic disease burden PCPCHs, suggesting that lower chronic disease burden PCPCHs may be associated to greater ED and IP savings. This insight is important for policy-related decisions in PCPCHs.

Specifically, to reduce short-term ED and IP expenditures in PCPCHs, policies must focus on the distribution of chronic conditions among patients in PCPCHs. The implementation of policies that focus on the engagement of a broader pool of patients in $\mathrm{PCPCH}$ or policies that encourage $\mathrm{PCPCH}$ clinics to attract and engage a broader pool of chronic conditions patients, instead of policies that emphasize focusing on high chronic disease burden patients, could improve the relative effectiveness of the model's application to reduce downstream health care utilization and expenditures. Policies for aligning payer incentives in $\mathrm{PCPCHs}$ to reduce inappropriate use (overuse and misuse) of ED and IP and expenditures can be improved based on the results of this research. The results suggest that incentives that narrowly focus on high chronic disease burden patients or higher average chronic disease burden PCPCHs may not achieve the expected short-term decreases in average ED and IP utilization and expenditures because the savings accrue from engaging a low chronic disease burden mix of patients. Payment policies that focus on high chronic disease burden patients could have unintended consequences, such as high chronic disease burden PCPCHs appearing ineffective in the short term while low chronic disease burden PCPCHs appear very effective in the short term. Such approaches to the design of 
payment policies could create adverse incentives for high chronic disease burden PCPCHs to focus on engaging low chronic disease burden patients for short-term benefits.

The use of differential payment incentives could help reduce these unintended effects. Such payment policies could use differential payment scales, for example, rewarding clinics that achieve savings from engaging low chronic disease burden patients less than clinics that achieve savings from engaging high chronic disease burden patients. PCPCH payment incentives should be risk-adjusted. The use of various risk-adjusted payment mechanisms can help improve the value and efficiency of care, reduce the incentives for attracting and engaging low chronic disease burden patients, and increase the average chronic disease burden of $\mathrm{PCPCH}$ clinics. Incentive policies that focus on broadening the chronic disease burden mix of PCPCHs specifically, and all Medical Homes in general, could also help reduce average ED and IP expenditures and discourage potential active-risk selection practices.

Proponents of the model expected that high chronic disease burden patients would be engaged in PCPCHs because of the patient-centered approaches to coordinating and integrating care for high chronic disease burden patients (Peikes et al., 2011; Rittenhouse, Thom, \& Schmittdiel, 2010). The results of this research, however, suggest that average chronic disease burden levels in PCPCHs did not change after $\mathrm{PCPCH}$ recognition, implying that $\mathrm{PCPCHs}$ are not changing the types of patients they engage. This result has broad policy implications in terms of the types of policies to enact, and the ability of such policies to influence or change where 
people seek their health care services. At the patient level, policies to educate high chronic disease burden patients about the benefits of PCPCH care, resources to guide such patients on how to identify and choose PCPCHs, and incentives to encourage specifically high chronic disease burden patients (and all patients in general) to enroll in PCPCHs may be important.

At the provider level, educating patients about seeking care in PCPCHs and the benefits of such care, using patient navigators to help engage and enroll patients, and sponsoring health promotion campaigns in the community locations of PCPCHs may help identify and enroll a broader mix of chronic care patients in PCPCHs. At the system level, recognizing more primary care clinics as PCPCHs across different regions of the state and providing more technical assistance on $\mathrm{PCPCH}$ attestation to support smaller primary care clinics that are either going through the process of PCPCH recognition or want to become PCPCHs will help improve access and provide more choices for patients. Additionally, policies to reduce co-pays or deductibles for high and low chronic disease burden patients who seek care in PCPCHs may be important for engaging a broader mix of chronic disease burden patients who can benefit from PCPCH care. Such policies would help engage more high chronic disease burden patients and lead to lower inappropriate ED and IP utilizations and expenditures.

The results on average ED and IP expenditures for high chronic disease burden PCPCHs raise the possibility of unobserved differences in the environments of high chronic disease burden patients in PCPCHs. The characteristics of high 
chronic disease burden patients in PCPCHs suggest that such unobserved differences may be based on the higher social determinants of health and related factors (shown figure 3.1) that exists in the environment for higher chronic disease burden patients in PCPCHs. Policies and incentives to support PCPCHs' approach to care may need to be specifically tailored to the types of PCPCHs, the communities where they operate, and the patients they engage because high average chronic disease burden PCPCHs may need additional or different support systems than low chronic disease burden PCPCHs.

\section{Recommendations for Further Research}

This research evaluated the levels of chronic condition-based health risks in PCPCHs to determine the engagement of patients who have high health risks in PCPCHs and to determine whether high average chronic disease burden PCPCHs achieve more savings from decreases in average ED and IP expenditures. The results imply that the distribution of chronic conditions of patients in PCPCHs is important and related to savings in ED and IP expenditures, but in different ways than expected. Further research to understand the application of PCPCHs and the relative effectiveness of the applications of the model will be needed.

While the chronic disease burden in PCPCHs did not change after the recognition of clinics as $\mathrm{PCPCHs}$, the proportion of other behavioral health patients in PCPCHs was low compared to the community. Since PCPCHs are designed to coordinate and integrate care for physical and behavioral health patients as part of delivering comprehensive primary care services, the results indicating that the 
proportion of other behavioral health patients in PCPCHs was significantly lower than the community are important. Research to determine the characteristics of such patients, their care preferences, and reasons they are not engaged in PCPCHs will provide insights into appropriate ways to engage such patients in PCPCHs. Such research can also help inform how PCPCHs can effectively engage other chronic condition patients who can benefit from the PCPCH approach to coordinating and integrating primary care services upstream.

Since coordinated care organizations (CCOs) were expected to mobilize and direct patients to receive coordinated and integrated care in $\mathrm{PCPCH}$, further research on patients' health care preferences, especially high chronic disease burden patients, may help improve existing knowledge on patient engagement, the preferences of such patients for PCPCH care, the different systems and mechanisms for engaging such patients, and the relative effectiveness of existing systems for engaging patients.

The focus of this research on the levels of chronic conditions in PCPCHs, based on their patients, assumed the measurement of chronic disease burden would be the same at the $\mathrm{PCPCH}$ practice level and the individual patient level; therefore, aggregating chronic conditions in PCPCHs would be adequate. Since different PCPCHs have different proportions of patients, though, their chronic disease burden may be different at the PCPCH and individual patient levels. The results also suggest that there may be unobserved heterogeneity in chronic disease burden levels. For example, the characteristics of high chronic disease burden patients, i.e. more Medicaid patients and of varying ages, suggest that high average chronic disease 
burden PCPCHs may be different based on unobserved differences in the higher social determinants of health for the patients who receive care in high average chronic disease burden PCPCHs. These unmeasured differences may have impacted the results on average ED and IP expenditures for high average chronic disease burden PCPCHs. Further research focus on measuring these unobserved differences and evaluating their differential effects on the PCPCH approach to care as well as ED and IP utilization and expenditures will help improve existing knowledge on the applications of the model for this population group.

The results also suggest the need for further studies into the classifications of appropriate and inappropriate care for high and low chronic disease burden patients in PCPCHs. From the results, savings from average ED and IP expenditures were comparatively greater for low average chronic disease burden patients in PCPCHs, but the expectation was that savings would be greater for higher average chronic disease burden patients in PCPCHs. Further research on what constitutes appropriate and inappropriate care in the receipt of primary care services by high and low chronic disease burden patients, and how changes in the receipt of such health care services translates into downstream decreases in ED and IP use and savings, will be important. Future research can also focus on measuring the differences in social determinants of health and other potentially unmeasured factors that affect the engagement of patients in PCPCHs and savings from ED and IP expenditures. The results of such research will help improve existing knowledge on the model as well as our understanding of 
the relative effectiveness of the model's application in the current delivery system environment.

A reflection on the methods used and the unexpected research results suggests the use of other methods to determine whether they provide different results. For example, the use of different comparators could provide different contexts for interpreting the research results. The comparison of high average chronic disease burden PCPCHs to low average chronic disease burden PCPCHs (to determine relative decreases in average ED and IP expenditures by the different chronic disease burden groups) suggests some misunderstanding of the trajectories of care transformation for high and low chronic disease burden patients in PCPCHs. For high chronic disease burden patients in PCPCHs, the results that ED and IP expenditures did not change may be preferable in terms of inappropriate utilization.

The results on higher savings from low average chronic disease burden patients further suggest that low chronic disease burden patients in PCPCHs may have more inappropriate use of ED and IP than expected. Using different comparators, such as comparing high average chronic disease burden $\mathrm{PCPCH}$ to comparable high average chronic disease burden comparators rather than comparing high and low average chronic disease burden $\mathrm{PCPCH}$ practices, may provide better contexts for the results and contribute to existing knowledge on what constitutes appropriate and inappropriate ED and IP utilization for high average and low average chronic disease burden patients in PCPCHs. Such research will also improve existing 
knowledge by helping to clarify the use of and accounting for ED and IP utilization and expenditures in PCPCHs.

The focus of this research on chronic conditions in PCPCHs assumed that the delivery of comprehensive primary care services in PCPCHs for high chronic disease burden patients (upstream care) would translate into increased primary care use, increased primary care expenditures upstream, and savings from reduced ED and IP utilization downstream. However, the results suggested that ED and IP use and expenditures for high chronic disease burden patients in PCPCHs remained flat, while primary care use and expenditures did not change. Since chronic care patients have complex health care needs and may interact differently with different sections of the delivery system, including their need for and benefits from referrals to specialty care, evaluating the use and expenditures for specialty care services by high and low chronic disease burden PCPCH patients could provide a broader context for interpreting the research results. Chronic care patients in PCPCHs may need and use chronic disease management, and it is expected that effective chronic disease management in PCPCHs could reduce their need and use of specialty care services. Insights into the use and expenditures for specialty services by chronic care patients in PCPCHs could provide another dimension for contextualizing the results of this research as well as evaluating the relative effectiveness of chronic disease management in PCPCHs.

The design and use of a chronic disease burden measure for this research was intended to additionally help determine whether the chronic conditions of PCPCH 
patients was a stable measure for evaluating the relative effectiveness of the model's application. The results suggesting the distribution of chronic conditions in PCPCHs is important and related to outcomes provide a foundation for further exploratory research into the relative effectiveness of the different applications of PCPCHs, for example, using the chronic disease burden measure for evaluating the distribution of chronic conditions in different PCPCH tier practices, different PCPCH geographic locations and regions, and different specialties of the model's application. Such evaluations could provide further insights into the different applications of the PCPCH model, the levels of chronic conditions, and the relative effectiveness of the different applications as well as the process to appropriately align payment incentives to increase the engagement of a broader mix of chronic care patients in the different applications of the model.

While the design and use of the chronic disease burden measure provided flexibility to stratify PCPCH clinics into high and low groups to determine utilization and expenditures by the high and low groups, the different results on ED and IP use and expenditures compel the need for further insights on the chronic disease burden measure to determine the potential weight of each of the ten markers of chronic conditions in the chronic disease burden measure and the strength of association between the markers of chronic conditions and ED and IP use and expenditures. Such insights could be useful for aligning payment incentives for PCPCHs to improve care for patients whose specific chronic conditions predispose them to increased ED and IP utilization and expenditures. 
The design of this research can be improved in other areas as well. The use of different health risk measures that are more comprehensive, can determine current and future health risks, reflect the appropriate utilization (reducing overuse and misuse) of ED and IP instead of the overall utilization of ED and IP; or the use of other measures that are more sensitive to ED and IP utilizations and expenditures may help to improve the results of this research. This research was designed as a shortterm relative effectiveness evaluation based on data constraints. The availability of more data over time can help undertake a more comprehensive longitudinal study to determine chronic disease burden levels in PCPCHs in the medium to long terms and their association to reducing ED and IP expenditures. 


\section{References}

Abrams, M. K., Davis, K., \& Haran, C. (2009). Can patient-centered medical homes transform health care delivery? Retrieved from (http://www.commonwealthfund.org/Content/From-the-President/2009/CanPatient-Centered-Meidcal -Home-Tranfrom-Health-Care-Delivery.aspx) on June 5, 2009.

Agency for Healthcare Research and Quality (AHRQ). (2011). National healthcare disparities report. Department of Health and Human Services publication \# 12-0006.

Altman, D (2015) How to think about higher growth in healthcare spending. The Wall Street Journal, retrieved from (http://www.blogs.wsj.com/washwire) on July 30, 2015.

Alexander, J. A., Hearld, L. R., Mittler, J. N., \& Harvey, J. (2012). Patient-physician role relationships and patient activation among individuals with chronic illness. Health Services Research, 47(3pt1), 1201-1223.

American Medical Association (2009) An introduction to risk assessment and risk adjustment models, retrieved from (http://www.ama-assn.org/go/pfp) on June 20, 2015.

Anderson, G. (2010). Chronic care: Making the case for ongoing care. Robert Wood Johnson Foundation. Retrieved from (http://www.rwjf.org) on October 10, 2011.

Ansari, Z., Carson, N., Serraglio, A., Baretti, T., \& Cicuttini, F. (2002). The Victorian ambulatory care sensitive conditions study: Reducing demand on hospital services in Victoria. Australian Health Review 25 (2): 71-77.

Ansari, Z., Laditka, J.N., \& Laditka, S.B. (2006). Access to health care and hospitalization for ambulatory care sensitive conditions. Medical Care Research and Review, 63:719 -741. 
Ansari, Z. (2007). The concept and usefulness of ambulatory care sensitive conditions as indicators of quality and access to primary health care. Australian Journal of Primary Health, 13(3):91-110.

Antos, J., Bertko, J., Chernew, M., Cutler, D., Goldman, D., McClellan, M....Shortell, S. (2009). Bending the curve: Effective steps to address long term health care spending growth. Engelberg Center for Health Care Reforms at Brookings. Retrieved from (http://www.brookings.edu/healthreform) on January 10, 2010.

Arrow, K. J. (1963). Uncertainty and the welfare economics of medical care, American Economic Review, 53(5): 941-973.

Ash, A. S., \& Ellis, R. P. (2012). Risk-adjusted payment and performance assessment for primary care. Medical care, 50(8), 643.

Ash, A.S., Randall, P.E., Pope, G.C., Ayanian, J.Z., Bates, D.W., Burstin, H....Yu,W. (2000). Using diagnosis to describe populations and predict costs. Health Care Financing Review, 21(3):7-28.

Asch, S.M., Baker, D.W., Keesey, J.W., Broder, M., Schonlau, M., Mayde, R.... Keeler, E. B. (2005). Does the collaborative model improve care for chronic heart failure Medical Care, 43(7):667-75.

Baicker, K., \& Chandra, A. (2004). Medicare spending, the physician workforce and beneficiaries' quality of care. Health Affairs, W4: 184 187. Retrieved from (http://www.content.healthaffairs.org/cgi/reprint/hlthaff.w4.pdf) on June 20, 2011.

Baseman, S., Boccuti, C., Moon, M., Griffin, S., Dutta, T (2016) Payment and Delivery System Reforms in Medicare: A Primer on Medical Homes, Accountable Care Organizations, and Bundled Payments. A Kaiser Family Foundation Report. Retrieved from (https://kaiserfamilyfoundation.files.wordpress.com/2016/02/8837-paymentand-delivery-system-reform-in-medicare1.pdf) on March 20, 2017. 
Basu, J., Friedman, B., \& Burstin, H. (2004). Managed care and preventable hospitalizations among Medicaid adults, Health Services Research, 39(3):489509.

Bebinger, M. (2014). Massachusetts patients can 'shop' for health care: at least in theory. Retrieved from (http://www.kaiserhealthnews.org/stories/2014/February/26) on March 3, 2014.

Behl-Chadha, B., Savageau, J. A., Bharel, M., Gagnon, M., Lei, P. P., \& Hillerns, C. (2017). Comparison of Patient Experience between a Practice for Homeless Patients and Other Practices Engaged in a Patient-Centered Medical Home Initiative. Journal of Health Care for the Poor and Underserved, 28(3): 11511164.

Berenson, R.A., \& Rich, E.C. (2010). U.S approaches to physician payment: The deconstruction of primary care, Society of General Internal Medicine, 25(6): 616-18.

Bernstein, J., Chollet, D., Peikes, D., \& Peterson,G. G. (2010). Medical Homes: Will they improve primary care? Issue brief by Mathematica Policy Research. Retrieved from (http://www.mathematica-mpr.com/health/series.asp) on June 25, 2010.

Berry, L.L., \& Mirabito, A.M. (2009). Innovative health care delivery. Science Direct, 53: 157-169.

Bertrand, M., Duflo, E., \& Mullainathan, S. (2001). How Much Should We Trust Difference-in-differences Estimates?” mimeograph. Massachusetts Institute of Technology, July 20, 2001.

Berwick, D. M., Nolan, T.W., \& Whittington, J. (2008). The Triple Aim: Care, health and cost. Health Affairs, 27(3): 25-35.

Berwick, D.M \& Hackbarth, A. D. (2012). Eliminating waste in U.S health care. Journal of American Medical Association, 207(14): E1-E4. 
Bielaszka-DuVernay, C. (2011). Vermont's blueprint for medical homes, community health teams, and better health at lower cost. Health Affairs, 30(3): 383-386.

Billings, J., Anderson, G.M., \& Newman, L.S. (1996). Recent findings on preventable hospitalizations. Health Affairs, 15:239-249.

Bindman, A., Grumbach, K., Osmond, D., Komaromy, M., Vranizan, K., Lurie, N..... Stewart, A. (1995). Preventable hospitalizations and access to health care. Journal of American Medical Association 274 (4): 305-11.

Bipartisan Policy Center (2012) What is driving U.S health care spending? America's unsustainable health care cost growth. Retrieved from (http://bipartisanpolicy.org/wpcontent/uploads/sites/default/files/BPC\%20Health.202012.pdf) on January 12, 2014.

Bitton, A., Martin, C., \& Landon, B. E. (2010). A nationwide survey of Patient Centered Medical Home demonstration projects. Society of General Internal Medicine, 25(6):584-592.

Bodenheimer, T., Lorig, L.K., Holman, H., \& Grumbach, K. (2002). Patient selfmanagement of chronic disease in primary care. Journal of the American Medical Association, 288 (19): 2469-75.

Bodenheimer, T., MacGregor, K., \& Sharifi, C. (2005). Helping patients manage their chronic conditions. California Health Care Foundation. Retrieved from (http://www.chcf.org.reports) on January 20, 2012.

Bodenheimer, T., Chen, E., \& Bennett, H. D. (2009). Confronting the growing burden of chronic disease: Can the U.S health care workforce do the Job. Health Affairs, 28(1):64-74.

Bott, D. M., Kapp, M. C., Johnson, L.B., \& Magno, L.M. (2009). Disease management for chronically ill beneficiaries in traditional Medicare. Health Affairs, 28(1):86-98.

Blass, J.C., \& Weksler, M. E. (1983). Towards an effective treatment of Alzheimer's disease. Annals of Internal Medicine, 98:251-52. 
Brown, R., Peikes, D \& Chen, A (2007) The Evaluation of the Medicare Coordinated Care Demonstration: Findings from the first two years, Mathematica Policy Research. Retrieved from (http://www.mathematicampr.com/health/series.asp) on June 25, 2009.

Buckley, J., \& Shang, Y. (2003). Estimating policy and program effects with observational data: The "difference-in-difference" estimator. Practical Assessment Research and Evaluations, 8(24). Retrieved from (http://wwwPAREonline.net/getvn=24) on January 20, 2013.

Cabral, L., Sefton, L., \& Anderson, T (2013) Early findings from the Massachusetts patient centered medical home initiative. Retrieved from (https://www.researchgate.net/publication) on February 23, 2018.

Caminal, J., Starfield, B., Sanchez, E., Casanova, C., \& Morales, M. (2004). The role of primary care in preventing ambulatory care sensitive conditions. European Journal of Public Health, 14: 246-51

Cassalino, P.L. (2005). Disease management and the organization of physician practices. Journal of American Medical Association, 293(4):485-88.

Center for Disease Control and Prevention (2015) Leading causes of death and numbers of deaths by sex, race, and Hispanic origin: United States, 1980 \& 2014. Health, United States, 2015. Retrieved from (https://www.cdc.gov/nchs/data/hus/hus15.pdf\#019) on January 20, 2016.

CMS (2003) A guide to implementing a health-based risk-adjustment payment system for Medicaid managed care programs. Submitted by the Center for Health Program Development and Management. Retrieved from (http://www.chpdm.org/publications/risk\%20adjustment\%20manual\%20appe ndices.pdf) on March 20, 2013.

Center for Financing, Access, and Cost Trends, AHRQ Household Component of the Medical Expenditure Panel Survey 2012 in Cohen, S.B (2014) Statistical Brief \#448: Differentials in the Concentration of Health Expenditures across Population Subgroups in the U.S., 2012. Retrieved from 
(https://meps.ahrq.gov/data_files/publications/st448/stat448.shtml) on December 20, 2016.

Chassin, M.R. (1998). The urgent need to improve health care quality: Institute of Medicine national roundtable on health care quality. Journal of American Medical Association, 280: 1000 -005.

Chin, M.H., Drum, M.L., Guillen, M., Rimington, A., Levie, J.R...... Schaefer, C.T. (2007). Improving and sustaining diabetes care in community health centers with the health disparities collaborative. Medical Care, 45(12): 1135-1143.

Cilliers, P. (1998). Complexity and post modernism: Understanding complex systems. Routledge, London.

Claxton, G., Cox, C., Damico, A., Levitt, L., \& Pollitz, K. (2016). Pre-existing conditions and medical underwriting in the individual insurance market prior to the ACA. Menlo Park, CA: Kaiser Family Foundation.

Cohen, J.T., Neumann, J.P., \& Weinstein, M.C. (2008). Does preventive care save money: Health economics and the presidential candidates. New England Journal of Medicine, 358(7):661-64.

Collaborative, P. C. P. C. (2007). Joint principles of the patient-centered medical home. Washington DC: Patient Centered Primary Care Collaborative.

Coleman, K., Austin, B.T., Brach, C., \& Wagner, E. (2009). Evidence on the chronic care model in the new millennium. Health Affairs, 28(1):75-85.

Congressional Budget Office. (2004). An analysis of the literature on disease management programs, Washington, D.C.

Congressional Budget Office. (2012). Implications of rising health care costs: Presentation to the Heritage Foundation by Bilheimer, L.T. Retrieved from (http://www.cbo.gov/sites/default/files/cbofiles/attachments/HeritagePresentat ion.pdf) on January 30, 2013.

Conwell, L.J., \& Cohen, J. W. (2002). Characteristics of people with high medical expenses in the U.S non-institutionalized population, in Hall, M.A. (2011). 
Cooley, C.W., McAllister, J.W., Sherrieb, K., \& Kuhlthau, K. (2009). Improved outcomes associated with medical home implementation in pediatric primary care. Pediatrics, 124:358 -364.

Cowling, T.E., Cecil, E.V., Soljak, M.A., Lee, J.T., Millett, A.M., Wachter, R.M .... Harris, M.J. (2013). Access to primary care and visits to emergency departments in England: A cross-sectional, population-based study. PLos ONE 8(6): e666991-e666996. Retrieved from (http://www.plosone.org) on July 20, 2013.

Crabtree, B.F., McDaniel, R., Miller, W.L., \& Stange, K. (1998). Understanding change in primary care practices using complexity theory. Journal of Family Practice, 46(5): 369-381.

Crabtree, B.F., Nutting, P.A., Miller, W. L., McDaniel, R. R., Stange, K.C., Jaen, C.R., \& Stewart, E. (2011). Primary care practice transformation is hard work: Insights from a 15-year developmental program of research, Medical Care, 49(12): S28-S35.

Culler, S. D., Parchman, M. L., \& Przybylski, M. (1998). Factors related to potentially preventable hospitalizations among elderly. Medical Care, 36(6):804-817.

Cumming, R.B., Knutson, D., Cameron, B.A., \& Derrick, B. (2002). A comprehensive analysis of claims-based methods of health risk assessment for commercial populations. Society of Actuaries. Retrieved from (http://www.soa.org) on July10, 2013.

Currie, J., \& Grogger, J. (2002). Medicaid expansions and welfare contractions: offsetting effects on prenatal care and infant health. Journal of Health Economics, 21(2): 313-335.

Davey-Smith, G., \& Lynch, J. (2004). Commentary: Social capital, social epidemiology and disease etiology. International Journal of Epidemiology, 33:691-700. 
David, G., Gunnarsson, C., Saynisch, P. A., Chawla, R., \& Nigam, S. (2015). Do Patient-Centered Medical Homes Reduce Emergency Department Visits? Health Services Research, 50(2): 418-39.

Davis, K. (2007). Paying for care episodes and care coordination. The New England Journal of Medicine, 356 (11):1166-68.

Davis, K., Schoen, C., \& Stremikis, K. (2010). Mirror, mirror on the wall: How the performance of U.S health care system compares internationally. The Commonwealth Fund Publication \#1400.

Devers, K. \& Berenson, R. (2009). Can accountable care organizations improve the value of health care by solving the cost and quality quandaries? Urban Institute.

Diedhiou, A., Probst, J.C., Hardin, J.W., Martin, A.B., \& Xirasager, S. (2010). Relationship between presence of a reported medical home and emergency department use among children with asthma. Medical Care Research and Review, 67:450-475.

DiLia, D. (2003). Distributional issues in the analysis of preventable hospitalizations. Health Services Research, 38(6):1761-79.

Donaldson, M. S., Yordy, K. D., Lohr, K. N., \& Vanselow, N. A. (1996). IOM Committee on the Future of Primary Care. Primary care - America's health in a new era Washington, DC: National Academy Press.

Dorr, D. A., Wilcox, A. B., Brunker, C. P., Burdon, R. E., \& Donnelly, S. M. (2008). The Effect of Technology-Supported, Multi-disease Care Management on the Mortality and Hospitalization of Seniors. Journal of the American Geriatrics Society, 56(12): 2195-02.

Dudley, A. R., Medlin, C. A., Hammann, L.B., Cisternas, M.G., Brand, R., Rennie, D. J., \& Luft, H.S. (2003). The best of both worlds. Potential of hybrid prospective/concurrent risk adjustment. Medical Care, 41(1):56-69.

Dzau, V.J., McClellan, M., Burke, S., Coye, M.J., Daschle, T.A., Diaz, A............. Zerhouni, E (2017) Vital Directions for Health and Health Care: Priorities 
from a National Academy of Medicine Initiative, National Academy of Medicine. Retrieved from (http://www.NAM.edu/Perspectives.pdf) on March 21, 2017.

Eby, D. (2009). Medical Home: The triple aim Institute of Healthcare Improvement, IHI Medical Home Work Group Document. Retrieved from (http://www.hss.state.ak.us/healthcommission/meetings/200908/pdf/medicalh ome.pdf) on January 20, 2011.

Eddy, D.M., Schlessinger, L., \& Kahn, R. (2005). Clinical outcomes and costeffectiveness of strategies for managing people at high risk for diabetes. Annals of Internal Medicine,143:251-264.

Ellis, R.P., \& McGuire, T. G. (1986). Provider behavior under prospective reimbursement: Cost sharing and supply. Journal of Health Economics, 5(2):129-51.

Enthoven, A.C., Crosson, F.J., \& Shortell, S.M. (2007). Redefining health care: Medical homes or archipelagos to navigate. Health Affairs, 26(5):1366 -72.

Epstein, A.J. (2001). The role of public clinics in preventable hospitalizations among vulnerable populations. Health Services Research, 32(2):405-20.

Epstein, R.M., Fiscella, K., Lesser, C.S., \& Stange, K.C. (2010). Why the nation needs a policy push on patient-centered health care, Health Affairs, 29(8): 1489-95.

Ettner, S.L., Frank, R.G., McGuire, T.G., \& Hermann, R.C. (2001). Risk adjustment alternatives in paying for behavioral health care under Medicaid. Health Services Research, 36(4):793-811.

Falik, M., Needleman, J., Wells, B. L., \& Kerb, J. (2001). Ambulatory care sensitive hospitalizations and emergency visits: Experiences of Medicaid patients using federally qualified health centers. Medical Care, 39(6):551-61.

Falik, M., Needleman, J., Herbert, R., Wells, B., Politzer, R., \& Benedict, M.B. (2005). Comparative Effectiveness of health centers as regular sources of 
care: Application of sentinel ACSC events as performance measures. Journal of Ambulatory Care Management, 29(1):24-35.

Feldstein, P.J. (2005). Health Care Economics, 6th Edition. New York, Thomson Delmar Learning.

Ferrante, J. M., Balasubramanian, B. A., Hudson, S. V., \& Crabtree, B. F. (2010). Principles of the patient-centered medical home and preventive services delivery. The Annals of Family Medicine, 8(2):108-16.

Ferrante, J. M., Cohen, D. J., \& Crosson, J. C. (2010). Translating the patient navigator approach to meet the needs of primary care. The Journal of the American Board of Family Medicine, 23(6): 736-44.

Fields, D., Leshen, E., \& Patel, K. (2010). Analysis and commentary on driving quality gains and cost savings through adoption of medical homes. Health Affairs, 29(5):819-26.

Fireman, B., Bartlett, J., \& Selby, J. (2004). Can disease management reduce health care costs by improving quality? Health Affairs, 23(6):63-75.

Fisher, E. S. (2008). Building a medical neighborhood for the medical home. New England Medical Journal of Medicine, 359(12).

Fishman, P.A., Goodman, M.J., Hornbrook, M.C., Meenan, R.T., Bachman, D, J., \& Rosetti, C.O. (2003). Risk adjustment using automated ambulatory pharmacy data: The Rx risk model. Medical Care, 41(1):84-99.

Flottemesch, T.J., Fontaine, P., Asche, S., \& Solberg, L. (2011). Relationships of clinical medical home scores and health care costs. Journal of Ambulatory Care Management, 34(1):78-9.

Flottemesch, T.J., Anderson, L.H., Solberg, L., Fontaine, P., \& Asche, S. (2012). Patient-Centered Medical Home cost reductions limited to complex patients. American Journal of Managed Care, 18(11): 667-89.

Fontaine, P., Flottemesch, T. J., Solberg, L. I., \& Asche, S. E. (2011). Is consistent primary care within a patient-centered medical home related to utilization 
patterns and costs? The Journal of Ambulatory Care Management, 34(1):1019.

Franks, P., Clancy, C.M., \& Nutting, P.A. (1992). Gate keeping revisited - protecting patients from overtreatment. New England Journal of Medicine, 327: 424-29.

Frank, R.G., McGuire, T.G., \& Newhouse, J. P. (1995). Risk contracts in managed mental health care. Health Affairs, 14(3): 50-64.

Frank, R.G., Glazer, J., \& McGuire, T. G. (2000). Measuring adverse selection in managed health care. Journal of Health Economics, 19(2000):829-54.

Franks, P., Cameron, C., \& Bertakis, K.D. (2003). On being new to an insurance plan: Health care use associated the first years in a health insurance plan. Annals of Family Medicine, 1:156-161.

Friedberg, M.W., Schneider, E. C., Rosenthal, M. B., Volpp, K. G., \& Werner, R.M. (2014). Association between participation in a multi-payer medical home intervention and changes in quality, utilization, and cost of care. Journal of American Medical Association, 311(8):815-25.

Fries, J. F. (1983). The compression of morbidity. Milbank Quarterly, 61(3):397-419.

Gelmon, S., Wallace, N., Sandberg, B., Petchel, S., Bouranis, N (2016) Implementation of Oregon's PCPCH Program: Exemplary Practice and Program Findings. Retrieved from (http://www.oregon.gov/OHA/HPA/CSIPCPCH/Documents/PCPCH-Program-Implementation-Report-Final-Sept2016.pdf) on October 20, 2016.

Getzen, T. E (2014) Macroeconomics dynamics of health: Lags and variability in mortality, employment, and spending. In Culyer, A. J (ed.) Encyclopedia of Health Economics, 2(1):165-176.Oxford, MA: Elsevier.

Gilfillan, R. J., Tomcavage, J., Rosenthal, M. B., Davis, D. E., Graham, J., Roy, J. A., ... \& Weikel, K. M. (2010). Value and the medical home: effects of transformed primary care. The American Journal of Managed Care, 16(8): 607-14. 
Gilmer, T., Kronick, R., Fishman, P., \& Ganiats, T.G. (2001). The medical Rx model: Pharmacy-based risk adjustment for public programs. Medical Care, 39(11):1188-202.

Glasgow, R.E., Orleans, C.T., Wagner, E.H., Curry, S.J., \& Solberg, L.I. (2001). Does the chronic care model serve also as a template for improving prevention? The Milbank Quarterly, 79(4):579-612.

Glasgow, R.E., Funnell, M.M., Bonomi, A.E., Davis, C., Beckham, V., \& Wagner, E.H. (2002). Self-management aspects of the improving chronic illness care breakthrough series: Implementation with diabetes and heart failure teams. Annals of Behavioral Medicine, 24(2):80-87.

Glazer, J., \& McGuire, T. (2000). Optimal risk adjustment in market with adverse selection: An application to managed care. American Econometric Review, 90(4):1055-71.

Goetzel, R.Z., Anderson, D.R., Whitmer, R.W., Ozminkowski, R.J., Dunn, R.L., Wassserman, J., \& The Health Enhancement Research Organization [HERO] (1998). Research Committee, Journal of Occupational \& Environmental Medicine, 40(10):843-54

Goetzel, R.Z. (2009). Do prevention or treatment services save money: The wrong debate? Health Affairs, 28(1): 37-45.

Goodman, D. C., Mick, S. S., Bott, D., Stukel, T., Chang, C. H., Marth, N., ... \& Carretta, H. J. (2003). Primary care service areas: a new tool for the evaluation of primary care services. Health Services Research, 38(1p1):287309.

Goodman, D.C., \& Grumbach, K. (2008). Does having more physicians lead to better health system performance? Journal of American Medical Association, 299 (3): 335-37.

Graves, B. (2012). To save money on health care, Oregon Health Plan looks to trim hospital, emergency room visits. The Oregonian. Retrieved from (http://www.oregonlive.com/health/index) on March 20, 2012. 
Grumbach, K., \& Bodenheimer, T. (2004). Can health care teams improve primary care practice? Journal of American Medical Association, 291:1246-51.

Grumbach, K., \& Grundy, P. (2010). Outcomes of implementing Patient-Centered Medical Home interventions: A review of the evidence from prospective evaluation studies in the U.S. Retrieved from (http://www.pcpcc.net/guide/benefits-implementing-primary-care-medicalhome) on June 10, 2010.

Gunterman, S. (2007). Enhancing value in Medicare: Chronic care initiatives to improve the program. Testimony to the U.S Senate special committee on aging. May 9, 2007. Commonwealth Fund. Retrieved from (http://www.commonwealthfund.org) on January 20, 2012.

Gunterman (2011). In Towards a Shared Vision of Payment Reform. Rosenthal, M.B. (2011). Massachusetts Medical Society, Harvard Scholl of Public Health, Commonwealth Fund Report. Retrieved from (http://www.commonwealthfund.org) on March 10, 2011.

Guy, D., Gunnarsson, C., Saynisch, P., Chawla, R., \& Nigam, S. (2013). Do PatientCentered Medical Homes reduce emergency department visits. Retrieved from (http://www.jhsph.edu/academics/degree-programs in-healtheconomics/shesg/david_guy.pdf) on February 20, 2014.

Harbrecht, M.G., \& Latts, L. M. (2012). Colorado's Patient-Centered Medical Home pilot met numerous obstacles yet saw results such as reduced hospital admissions. Health Affairs, 31(9):2010-17.

Health Maintenance Associates. (2012). Financial model and analysis of potential statewide savings from statewide adoption of the CCO health care delivery system redesign. Retrieved from (http://www.oregon.gov/oha/legactivity/2012/hma-report.pdf) on May 28, 2013. 
Healthy People (2020) Determinants of Health. Retrieved from (https://www.healthypeople.gov/2020/about/foundation-healthmeasures/Determinants-of-Health) on March 1, 2018.

Hearld, L. R., \& Alexander, J. A. (2012). Patient-centered care and emergency department utilization: a path analysis of the mediating effects of care coordination and delays in care. Medical Care Research and Review, 69(5): 560-80.

Hebert, P. L., Liu, C. F., Wong, E. S., Hernandez, S. E., Batten, A., Lo, S., ... Fihn, S. D. (2014). Patient-centered medical home initiative produced modest economic results for Veterans Health Administration, 2010-12. Health Affairs (Project Hope), 33(6): 980-87.

Hester, J. (2010). Designing Vermont's pay-for-population health system. Preventing Chronic Disease, 7(6):1-6. Retrieved from (http://www.cdc.gov/pcd/issues/2010/) on March 15, 2011.

Higgins, A., Zeddies, T., \& Pearson, S. D. (2011). Measuring the performance of individual physicians by collecting data from multiple health plans: The results of a two-state test. Health Affairs, 30 (4):673-81.

Hing, E., Kurtzman, E., Lau, D. T., Taplin, C., \& Bindman, A. B. (2017). Characteristics of Primary Care Physicians in Patient-centered Medical Home Practices: United States, 2013.

Hoffman, C., \& Paradise, J. (2008). Health insurance and access to health care in the United States. Annals of the New York Academy of Sciences, 1136(1):149-60.

Holden, L.M. (2005). Complex adaptive systems: Concept analysis. Journal of Advanced Nursing, 52(6):651-57.

Holman, H., \& Lorig, K. (2000). Patients as partners in managing chronic disease. British Medical Journal, 320:526 -27.

Homer, C.J., Forbes, P., Horvitz, L., Peterson, L.E., Wypij, D., \& Heinrich, P. (2005). Impact of a quality improvement program on care and outcomes for children 
with asthma. Journal of American Medical Association Pediatrics, 159(5):464-69.

Homer, C.J., \& Baron, R. J. (2010). How to scale up primary care transformation: What we know and what we need to know. Society of General Internal Medicine, 25(6):625- 29.

Horner, R.D (2012) Risk Adjusted capitation in an era of personalized medicine: A dangerous opportunity to bend the health care cost curve. Medical Care 50(8): 633-34.

Hornbrook, C. M., \& Goodman, M. J. (1996). Chronic disease, functional health status and demographics: A multi-dimensional approach to risk adjustment. Health Services Research, 31(3):283-307.

Horrocks, S., Anderson, E., \& Salisbury, C. (2002). Systematic review of whether nurse practitioners working in primary care can provide equivalent care to doctors. British Medical Journal, 324(7341):819-23.

McGinnis, P.B., Howk, S., Ong, E., \& Simeon, J (2016) Health Districts in Oregon, Oregon Rural Practice-based Research Network (ORPRN) White Paper. Retrieved from (http://www.ohsu.edu/xd/outreach/oregon-ruralhealth/data/publications/upload/2016-Health-District-White-Paper.pdf) on February 28, 2018.

Hroscikoski, M.C., Solberg, L.I., Sperl-Hillen, J.M., Harper, P.G., McGrail, M.P., \& Crabtree, B.F. (2006). Challenges of change: A qualitative study of chronic care model implementation. Annals of Family Medicine, 4(4):317-26.

Hsiao, W. C., Knight, A. G., Kappel, S., \& Done, N. (2011). What other states can learn from Vermont's bold experiment: embracing a single-payer health care financing system. Health Affairs, 30(7), 1232-41.

Huber, P. J. (1967). The behavior of maximum likelihood estimates under nonstandard conditions. In proceedings of the Fifth Berkeley Symposium on Mathematical Statistics and Probability (pp.221-233). Berkeley California. University of California Press. 
Huang, E.S., Zhang, Q., Sydney, E. S., Brown, M. L., Drum, D. O., \& Chin, H.M. (2007). The cost-effectiveness of improving diabetes care in U.S. federally qualified community health centers. Health Services Research; 42:6.

Institute of Medicine. (1999). To Err Is Human: Building a safer health system, Washington National Academy Press.

Institute of Medicine. (2001). Crossing the quality chasm: A new health system for the twenty-first century. Washington national academy press, Washington D.C.

Jackson, G. L., Powers, B. J., Chatterjee, R., Bettger, J. P., Kemper, A. R., Hasselblad, V., ... \& Gray, R. (2013). The patient-centered medical home: a systematic review. Annals of Internal Medicine, 158(3), 169-78.

Jackson, G. L., \& Williams, J. W. (2015). Does PCMH "Work"? The need to use implementation science to make sense of conflicting results. JAMA Internal Medicine, 175(8), 1369-70.

Jencks, S.F., Williams, M.V., \& Coleman, E. A. (2009). Re-hospitalizations among patients in the Medicare fee-for service program. The New England Journal of Medicine, 360(14):1418-28.

Johnson, M.L (2013) Risk Assessment and Adjustment: Adjusting for sick patients or sick system? Medical Care 41(1): 4-7.

Jones, C., Finison, K., McGraves-Lloyd, K., Tremblay, T., Mohlman, M. K., Tanzman, B., ... \& Samuelson, J. (2016). Vermont's community-oriented allpayer medical home model reduces expenditures and utilization while delivering high-quality care. Population Health Management, 19(3), 196-205.

Kaiser Family Foundation. (2012). Health care costs, a primer: Key information on health care costs and their impact. Retrieved from (http://www.kff.org/insurance/upload/7670-03.pdf) on February 5, 2013.

Kansagara, D., Englander, H., Salanitro, A., Kagen, D., Theobald, C., Freeman, M., \& Kripalani, S. (2011). Risk prediction models for hospital readmission: A 
systematic review. Journal of American Medical Association, 306(15):168898.

Kanton, W.J., Schoenbaum, M., Ming, F.Y., Callahan, M.C., Williams, J., Hunkeler, E. ...Unutzer, J. (2005). Cost effectiveness of improving primary care treatment of late life depression. Archives of General Psychiatry, 62: 1313-20.

Kaye, N., Buxbaum, J., \& Takach, M. (2011). Building medical homes: Lessons from eight states with emerging programs. National Academy for State Health Policy. Retrieved from (http://www.nashp.org/resources) on April 15, 2013.

Kernisan, L (2013) Patient Engagement: On metrics and meaning. The Health Care Blog. Retrieved from (http://thehealthcareblog.com/blog/2013/09/12/patientengagement-on-metrics-and-meaning) on September 8, 2014.

Kilo, C. M., \& Wasson, J. H. (2010). Practice redesign and the patient-centered medical home: history, promises, and challenges. Health Affairs, 29(5), 773 78.

Klitzner, T. S., Rabbitt, L. A., \& Chang, R. K. R. (2010). Benefits of care coordination for children with complex disease: a pilot medical home project in a resident teaching clinic. The Journal of Pediatrics, 156(6), 1006-10.

Koehler, B.E., Richter, K.M., Youngblood, L., Cohen, B.A., Prengler, I.D., Cheng, D., \& Masica, A.L. (2009). Reduction of 30-day post discharge hospital readmission or emergency department (ED) visit rate in high-risk elderly medical patients through delivery of a targeted care bundle. Journal of Hospital Medicine, 4(4):211-18.

Kogan, M. D., Alexander, G. R., Teitelbaum, M. A., Jack, B.W., Kotelchuck, M., \& Pappas, G. (1995). The effects of gaps in health insurance on continuity of a regular source of care among pre-school aged children in the United States. Journal of American Medical Association, 274:1429-35.

Kronick, R., Gilmer, T., Dreyfus, T., \& Lee, L. (2000). Improving health-based payment for Medicaid beneficiaries: CDPS. Health Care Financing Review, 21(3):29-63. 
Kuzel, A.J., \& Skoch, E. M. (2009). Achieving a patient-centered medical home as determined by the NCQA: At what cost and to what purpose. Annals of Family Medicine, 7(1):85-86.

Lee, C., \& Rogal, D. (1997). A special report on risk adjustment: A key to changing incentives in the health insurance market. Robert Wood Johnson Foundation. Retrieved from (http://www.rwjf.org) on March 30, 2012.

Leggetter, S., Chaturvedi, N., Fuller, J.H., \& Edmonds, M.E. (2002). Ethnicity and risk of diabetes-related lower extremity amputation: A population-based, casecontrol study of African Caribbean's and Europeans in the United Kingdom. Archives of Internal Medicine, 162(1):73-8.

Lemak, C. H., Nahra, T. A., Cohen, G. R., Erb, N. D., Paustian, M. L., Share, D., \& Hirth, R. A. (2015). Michigan's fee-for-value physician incentive program reduces spending and improves quality in primary care. Health Affairs, 34(4):645-52.

Lewis, V.A., McClurg, A.B., Smith, J., Fisher, E., \& Bynum, J. P. W. (2012). Attributing patients to Accountable Care Organizations: Performance year approach aligns stakeholders' interests. Health Affairs, 32(3):587-95.

Litaker, D., Tomolo, A., Liberatore, V., Stange, K.C., \& Aron, D. (2006). Using complexity theory to build interventions that improve health care delivery in primary care. Journal of General Internal Medicine, 21: S30-4.

Love, D., Custer, W., \& Miller, P. (2010). All-payer claims databases: State initiatives to improve health care transparency. The Commonwealth Fund, 1439(99):1-14.

Lubitz, J., Cai, L., Kramarow, E., \& Lentzner, H. (2003). Health, life expectancy, and health care spending among the elderly. New England Journal of Medicine, 349(11):1048-55.

Luft, H.S. (2009). Economic incentives to promote innovation in health care delivery. Clinical Orthopedics and Related Research, 467: 2497-505. 
Ma, C.T. A. (1994). Health Care Payment Systems: Cost and Quality Incentives, Journal of Econometric and Management Strategy, 3(1):93-112.

Maciejewski M.L., Liu C.F., Derleth A., McDonell M., Anderson S., and Fihn S.D (2005) The performance of administrative and self-reported measures for risk adjustment of Veterans Affairs expenditures. Health Services Research, 40(3):887-904.

Maeng, D. D., Graham, J., Graf, T. R., Liberman, J. N., Dermes, N. B., Tomcavage, J., ... \& Steele, J. G. (2012). Reducing long-term cost by transforming primary care: evidence from Geisinger's medical home model. The American journal of managed care, 18(3):149-55.

Maeng, D. D., Khan, N., Tomcavage, J., Graf, T. R., Davis, D. E., \& Steele, G. D. (2015). Reduced acute inpatient care was largest savings component of Geisinger Health System's patient-centered medical home. Health Affairs, 34(4), 636-44.

Manning, W.G (1998) The logged dependent variable, heteroskedasticity, and the retransformation problem. Journal of Health Economics, 17(3):283-95.

Marshall, M.N., Shekelle, P.G., Leatherman, S., \& Brook, R. H (2000). The public release of performance data. What do we expect to gain? A review of the evidence. Journal of American Medical Association, 283:1866-74.

Martin, J.C., Avant, R.F., \& Bucholtz, J. R. (2004). The Future of Family Medicine: A collaborative project of the Family Medicine community. Annals of Family Medicine, 2(1): S3-32.

Martin, K.E., Rogal, D.L., \& Arnold, S. B. (2004). Health-based risk assessment: Risk adjustment payments and beyond. Health Care Financing and Organization. Retrieved from (http://www.academyhealth.org/Publications) on July 20, 2013.

Mattke, S., Seid, M., \& Ma, S. (2007). Evidence for the effect of disease management: Is one billion a year a good investment. American Journal of Managed Care, 13: 670-76. 
McCall, N., Cromwell, J., \& \& Bernard, S. (2007). Evaluation of phase one of Medicare health support pilot program under traditional fee-for-service. CMC report to Congress, Triangle Park, NC.

McGrath, R. J., Laflamme, D. J., Schwartz, A. P., Stransky, M., \& Moeschler, J. B. (2009). Access to genetic counseling for children with autism, Down syndrome, and intellectual disabilities. Pediatrics, 124(Supplement 4), S44349.

Medicare Payment Advisory Commission. (2008). Report to Congress: Reforming the delivery system. Retrieved from (http://www.medpac.gov/documents/june_EntireReport.pdf) on June 12, 2008.

Merrill, C.T., Owens, P.L., \& Stocks, C. (2008). Emergency department visits for adults in community hospitals from selected states, 2005. Healthcare Cost and Utilization Project, Statistical Brief \# 47.

Meyer, B. D. (1995). Natural and quasi-experiments in economics. Journal of Business \& Economic Statistics, 13(2):151-161.

Meyer, H. (2010). Group Health's move to the medical home: for doctors, it's often a hard journey. Health Affairs, 29(5):844-51.

Minnesota Department of Health [MDH]. (2012). Health care homes: Annual report on implementation to the Minnesota legislature 2011. Retrieved from (http://www.health.state.mn.us/healthreform/homes/legreport/2011HCHLegR eport.pdf) on May 9, 2014.

Murty, S., Beglay, C.E., \& Swint, J.M. (2011). Inpatient preventable hospitalizations for ambulatory care sensitive conditions in Harris County. University of Texas Health Sciences Center, Houston.

National Committee on Quality Assurance [NCQA] (2014). NCQA recognition directory. Retrieved from (http://recognition.ncqa.org) on May 20, 2014. 
Nawar, E.W., Niska, R.W., \& Xu, J. (2007). National hospital ambulatory medical care survey: 2005 emergency department summary. National Center for Health Statistics \# 386, Hyattsville, MD.

Neal, J., Chawla, R., Colombo, C. M., Snyder, R. L., \& Nigam, S. (2015). Medical homes: cost effects of utilization by chronically ill patients. The American Journal of Managed Care, 21(1): e51-61.

Neff, J.M., Sharp, V.L., Muldoon, J., Graham, J., \& Myers, K. (2004). Profile of medical charges for children by health status group and severity level in a Washington State health plan. Health Services Research, 39(1):73-89.

Nelson, K. M., Helfrich, C., Sun, H., Hebert, P. L., Liu, C.-F., Dolan, E., ... Hernandez, S. E. (2014). Implementation of the patient-centered medical home in the Veterans Health Administration: Associations with patient satisfaction, quality of care, staff burnout, and hospital and emergency department use. JAMA Internal Medicine, 174(8):1350-58.

Newhouse, J.P. (1994). Economic analysis: Patients at risk, health reform and risk adjustment. Health Affairs, Spring (1):132-46.

Newhouse, J. P. (1996a). Reimbursing health plans and health providers: Efficiency in production versus selection. Journal of Economic Literature, XXXIV: 1236-63.

Newhouse, J. P. (1996b). Health reform in the United States. The Economic Journal, 106:1713-24.

Newhouse, J.P., Buntin, M.B., \& Chapman, J. (1997). Risk adjustment and Medicare: Taking a Closer Look, Health Affairs, 16(5): 26-43.

Nielsen, M., Langner, B., Zema, C., Hacker, T., \& Grundy, P. (2012). Benefits of implementing the PCMH: A review of cost \& quality results. Retrieved from (http://www.pcpcc.net/guide/benefits-implementing-primary-care-medicalhome) on October 20, 2012.

Nielsen, M., Buelt, L., Patel, K., Nichols, L. M., \& Fund, M. M. (2016). The patientcentered medical home's impact on cost and quality. Annual Review of 
Evidence: 2014-2015. Retrieved from (http://www.pcpccc.org) on November 13, 2017.

Nutting, P.A., Miller, W.L., Crabtree, B.F., Jaén, C.R., Stewart, E.E., \& Stange, K.C. (2009). Initial lessons from the first national demonstration project on practice transformation to a Patient-Centered Medical Home. Annals of Family Medicine, 7(3):254-60.

Oklahoma Health Care Authority. (2012). SoonerCare physician agreement for choice primary care providers. Retrieved from (http://www.okhca.org/pdf/physicianaddendum) on January 10, 2012.

O’Malley, A.S., Hoangmai, H.P., Schrag, D., Wu, B., \& Bach, P. B. (2007). Potentially avoidable hospitalizations for COPD and pneumonia: The role of physician and practice characteristics. Medical Care, 45(6):562-570.

Oregon Health Authority [OHA] (2010). Oregon's action plan for health. Retrieved from (http://www.oregon.gov/OHA/action-plan/rpt-2010.pdf) on January 10, 2011.

Oregon Health Authority [OHA] (2012a). Standards and measures for PatientCentered Primary Care Home: Final report. Retrieved from (http://www.dhcs.ca.gov/provgovpart/Documents/Waiver\%20Renewal/Final PCPCH_Report_2\%2023\%2012_(2).pdf) on January 24, 2013.

Oregon Health Authority [OHA] (2012b). Oregon public health division: State health profile. Retrieved from (http://public.health.oregon.gov/About/Documents/oregon-state-healthprofile.pdf) on January 31, 2013.

Oregon Health Authority [OHA] (2013). Oregon's health system transformation: Quarterly progress report. Retrieved from (http://www.oregon.gov/OHA/quarterly-progress/rpt-2013pdf) on May, 25, 2013.

Oregon Medicaid Advisory Committee (2017) Definition of the Social Determinants of Health for Oregon's Coordinated Care Organizations. Retrieved from 
(http://www.oregon.gov/oha/HPA/HP-

MAC/MACmeetings/Materials\%20Definition\%20of\%20the\%20Social\%20De terminants\%20of\%20Health\%20for\%20Oregon\%20CCOsV2.pdf) on March $1,2018$.

Oster, A., \& Bindman, A.B. (2003). Emergency department visits for ambulatory care sensitive conditions: Insights into preventable hospitalizations. Medical Care, 41(2):198-207.

Orszag, P.R., \& Ellis, P. (2007). Addressing rising health care costs - A view from the Congressional Budget Office. New England Journal of Medicine, 357(19): 1885-1887.

Ouwens, M., Wollershheim, H., Hermens, R., Hulscher, M., \& Grol, R. (2005). Integrated care programmes for chronically ill patients: A review of systematic reviews. International Journal for Quality in Health Care, 17(2):141-45.

Parchman, M.L., \& Culler, S. (1994). Primary care and avoidable hospitalizations. Journal of Family Practice, 39:123-28.

Park, E. (2011). Ensuring effective risk adjustment: An essential step for the success of the health insurance exchanges and market reforms under the Affordable Care Act. Center on Budget and Policy Priorities. Retrieved from (http://www.cbpp.org) on May 21, 2013.

Patient Protection and Affordable Care Act. (2010). Public Law 111-148 passed by the $111^{\text {th }}$ Congress of the United States. Retrieved from (http://democrats.senate.gov/reform/patient-protection-affordable-care-act-aspassed.pdf) on November 24, 2010.

Patient-Centered Primary Care Homes [PCPCH]. (2013). Patient-Centered Primary Care Home Program: Supplemental payment options for recognized clinics. Retrieved from (http://www.oregon.gov/oha/pcpch/payment-incentives.aspx) on May 15, 2014. 
Pearson, M.L., Wu, S., Schaefer, J., Bonomi, A.E., Shortell, S.M., Mendel, P.J...... Keeler, E.B. (2005). Assessing the implication of the chronic care model in quality improvement collaborative. Health Services Research, 40(4):978-96.

Paulus, R. A., Davis, K., \& Steele, G. D. (2008). Continuous innovation in health care: Implications of the Geisinger experience. Health Affairs, 27(5):1235-45.

Peikes, D., Dale, S., Lundquist, E., Genevro, J., \& Meyers, D. (2011). Building the evidence base for the medical home: What sample and sample size do studies need? Agency for Healthcare Research and Quality, Publication \#11-0100EF. Rockville MD.

Pines, J. M., Keyes, V., van Hasselt, M., \& McCall, N. (2015). Emergency department and inpatient hospital use by Medicare beneficiaries in patientcentered medical homes. Annals of Emergency Medicine, 65(6), 652-60.

Plesk, P. (2001). Redesigning health care with insights from the science of complex adaptive systems. In Institute of Medicine (Ed.), Crossing the quality chasm: A new health system for the 21st century, Washington DC: National Academy Press.

Plesk, P., \& Greenhalgh, T. (2001). The challenges of complexity in healthcare. British Medical Journal, 323:625-28.

Politzer, R.M., Yoon, J., Shi, L., Huges, R.G., Regan, J., \& Gaston, H. M. (2001). Inequality in America: The contribution of health centers in reducing and eliminating disparities in access to care. Medical Care Research and Review, 58:234-48.

Ralston, J. D., Rutter, C. M., Carrell, D., Hecht, J., Rubanowice, D., \& Simon, G. E. (2009). Patient use of secure electronic messaging within a shared medical record: a cross-sectional study. Journal of General Internal Medicine, 24(3), 349-55.

Randall, I., Mohr, D. C., \& Maynard, C. (2014). VHA Patient-Centered Medical Home Associated with Lower Rate of Hospitalizations and Specialty Care 
Among Veterans with Posttraumatic Stress Disorder. Journal for Healthcare Quality.

Raskas, R.S., Latts, L.M., Hummel, J.R., Wenners, D., Levine, H., \& Nussbauum, S. R. (2012). Early results show WellPoint's Patient-Centered Medical Home pilots have met some goals for cost, utilization and quality. Health Affairs, 31(9):2002-09.

Reid, R.J., Fishman, P.A., Yu, O., Ross, T. R., Tufano, J.T., Soman, M.P.... \& Larson, E.B. (2009). A Patient-Centered Medical Home demonstration: A prospective, quasi-experimental, before and after evaluation. American Journal of Managed Care, 15(9): e71-87.

Reid, R.J., Coleman, K., Johnson, E.A., Fishman, P.A., Hsu, C., Soman, M. P... \& Larson, E.B. (2010). The Group Health medical home at year 2: Cost savings, higher patient satisfaction and less burn outs for providers. Health Affairs, 29 (5):835-43.

Reinhardt, W. E. (1998). Can efficiency in health care be left to the market? Journal of Health Politics, Policy and Law, 26(5):967-91.

Rice, K. L., Dewan, N., Bloomfield, H. E., Grill, J., Schult, T. M., Nelson, D. B., ... \& Caldwell, M. (2010). Disease management program for chronic obstructive pulmonary disease: a randomized controlled trial. American Journal of Respiratory and Critical Care Medicine, 182(7):890-96.

Rich, M.W., Beckham, V., Wittenberg, C., Leven, C., Freedland, K.E., \& Carney, R. M. (1995). A multidisciplinary intervention to prevent the readmission of elderly patients with congestive heart failure. New England Journal of Medicine, 333:1190-95.

Rissi, J. J., Gelmon, S., Saulino, E., Merrithew, N., Baker, R., \& Hatcher, P. (2015). Building the foundation for health system transformation: Oregon's patientcentered primary care home program. Journal of Public Health Management and Practice, 21(1):34-41. 
Rittenhouse, D.R., Shortell, S.M., \& Fisher, E.S. (2009). Primary care and accountable care-two essential elements of delivery system reform. The New England Journal of Medicine, 361(24):2301-03.

Rittenhouse, D.R., Thom, D.H., \& Schmittdiel, J. A. (2010). Developing a policyrelevant research agenda for the Patient-Centered Medical Home: A focus on outcomes. Journal of General Internal Medicine, 25(6):593-600.

Robert Graham Center. (2007). The Patient Centered Medical Home: History, seven core features evidence and transformational change. Retrieved from (http://www.graham-center.org) on October 30, 2011.

Robinson, J.C., \& Luft, H.S. (1988). Competition, regulation and hospital costs. 1982-86, Journal of American Medical Association, 260:2676-81.

Robinson, J. C. (1997). Physician-hospital integration and economic theory of the firm. Medical Care Research and Review, 53:3.

Robinson, J.C. (2001). The end of managed care. Journal of American Medical Association, 285(20): 2622-28.

Roblin, D.W., Howard, D.H., Becker, E.R., Adams, E.K., \& Roberts, M.H. (2004). Use of midlevel practitioners to achieve labor cost savings in primary care practices of an MCO. Health Services Research, 39(3):607-25.

Rolando, L., Byrne, D.W., McGown, P.W., Goetzel, R.Z., Elasy, T.A., \&Yarbrough, M. I. (2013). Health risk factor modification predicts incidence of diabetes in an employee population: Results of an 8-year longitudinal cohort study. Journal of Occupational \& Environmental Medicine, 55(4):410-15.

Rosenthal, M.B., Abrams, M.K., \& Bitton, A. (2012). Recommended core measures for evaluating the Patient-Centered Medical Home: Costs, utilization and clinical quality. The Commonwealth Fund: 1601, 12.

Rosenthal, M. B., Alidina, S., Friedberg, M. W., Singer, S. J., Eastman, D., Li, Z., \& Schneider, E. C. (2016). A difference-in-difference analysis of changes in quality, utilization and cost following the Colorado multi-payer patient- 
centered medical home pilot. Journal of General Internal Medicine, 31(3):289-96.

Rosenbaum, P.R \& Rubin, D.B (1983) The central role of the propensity score in observational studies for causal effects. Biometrika, 70:41-55

Rosero-Bixby, L. (2004). Spatial Access to health care in Costa Rica and its Equity: A GIS-based study. Social Science and Medicine, 58:1271-84.

Rothkopf, J., Brookler, K., Wadhwa, S., \& Sajovetz, M. (2011). Medicaid patients seen at Federally Qualified Health Centers use hospital services less than those seen by private providers. Health Affairs, 30(7):1335-42.

Russell, L.B. (1986). Is prevention better than cure? Brookings Institution, Washington, D.C

Russell E. G., Orleans, T.C., Wagner, E.H., Curry, S.J., \& Solberg, L.I. (2001). Does the chronic care model serve also as a template for improving prevention? Milbank Quarterly, 79(4):579-12.

Russell, L.B. (2009). Preventing chronic disease: An important investment, but don't count on cost savings. Health Affairs, 28(1):42-45.

Safran, D.G., Tarlov, A.R., \& Rogers, W.H. (1994). Primary care performance in feefor-service and prepaid health care systems. Journal of American Medical Association, 271(20):1579-86.

Saha, S., Solotaroff, R., Oster, A., \& Bindman, A. B. (2007). Are preventable hospitalizations sensitive to changes in access to primary care? The care of the Oregon Health Plan. Medical Care, 45(8):712-19.

Savage, A. I., Lauby, T., \& Burkard, J. F. (2013). Examining selected patient outcomes and staff satisfaction in a primary care clinic at a military treatment facility after implementation of the patient-centered medical home. Military Medicine, 178(2):128-34.

Shen, Y., \& Ellis, R. P. (2002). How profitable is Risk Selection: A comparison of four risk adjustment models. Health Economics, 11:165-74. 
Shi, L. (1994). Primary care, specialty care and life chances. International Journal of Health Services, 24:431-58.

Shi, L., \& Lu, N. (2000). Individual socio-demographic characteristics associated with hospitalization for pediatric ambulatory care sensitive conditions. Journal of Health Care for the Poor and Underserved, 11(4):373-84.

Shi, L., \& Starfield, B. (2000). Primary care, income inequalities and self- rated health in the U.S: A mixed-level analysis. International Journal of Health Services, 30:541-55.

Shi, L., Macinko, B., Starfield, R., Politzer, J., Wulu, \& Xu, J. (2005). Primary care, social inequalities and all cause, heart disease and cancer mortality in U.S counties. American Journal of Public Health, 95:674-80.

Shi, L., \& Singh, D.A. (2008). Delivering health care in America: A systems approach, $4^{\text {th }}$ Edition, Jones and Bartlett Publishers: MA.

Sia, C., Tonniges, T.F., Osterhus, E., \& Toba. (2004). History of the medical home concept. Pediatrics, 113:1473 -78.

Smith, A (2011) Overview of the Medicaid health home care coordination benefit. National Council webinar. Retrieved from (https://www.openminds.com/market-intelligence/executivebriefings/040912-homes) on February 20, 2018.

Sochalski, J., Jaarsman, T., Krumholz, H.M., Laramee, A., McMurray, J.V., Naylor, M.D.... Stewart, S. (2009). What works in chronic care management: The case of heart failure. Health Affairs, 28(1):179-89.

Solberg, L.I., Crain, L.A., Sperl-Hillen, J.M., Hroscikoski, M.C., Engebretson, K.I., \& O’Connor, P.J. (2006). Care quality and implementation of the chronic care model: A quantitative study. Annals of Family Medicine, 4:310-16.

Sperl-Hillen, J. M., Solberg, L. I., Hroscikoski, M. C., Crain, A. L., Engebretson, K. I \& O'Connor, P. J. (2004). Do all components of the chronic care model contribute equally to quality improvement? Joint Commission Journal on Quality and Patient Safety, 30(6):303-09. 
Stange, K. C., Nutting, P. A., Miller, W. L., Jaén, C. R., Crabtree, B. F., Flocke, S. A., \& Gill, J. M. (2010). Defining and measuring the patient-centered medical home. Journal of General Internal Medicine, 25(6):601-12.

Starfield, B., \& Shi, L. (2004). The medical home, access to care, and insurance: A review of evidence, Pediatrics, 113:1493-98.

Starfield, B., Shi, L., \& Macinko, J. (2005). Contributions of primary care to health systems and health. Milbank Quarterly, 83(3): 457-502.

Starfield, B. (2011). Is patient-centered care the same as person-focused care? Permanente Journal. 2011 Spring, 15(2):63-9.

Steele, G. D., Haynes, J. A., Davis, D. E., Tomcavage, J., Stewart, W. F., Graf, T. R., ... \& Shikles, J. (2010). How Geisinger's advanced medical home model argues the case for rapid-cycle innovation. Health Affairs, 29(11):2047-53.

Steiner, B., Denham, A.C., Ashkin, E., Newton, W.P., Wroth, T., \& Dobson, L.A. (2008). Community care of North Carolina: Improving care through community health networks. Annals of Family Medicine, 6(4):361-67.

Stenger, R. J., Smith, J., McMullan, J.B., Rodriguez, G.S., Dorr, D.A., Minniti, M..... Saultz, J. S. (2012). Defining the medical home: The Oregon experience. Journal of Ambulatory Board of Family Medicine, 25:869-77.

Sylvia, M.L. (2008). Guided care: Costs and utilization outcomes in a pilot study. Disease Management, 11(1):29-36.

Takach, M., Gauthier, A., Sims-Kastelein., \& Kaye, N. (2010). Strengthening primary care and chronic care: state innovations to transform and link small practices. National Academy for State Health Policy, A Commonwealth Fund Report. Retrieved from (http://www.michigan.gov/documents/mdch/shstate.innovations.to_transform link_small_practices_346168_7.pdf) on February 2, 2015.

Takach, M. (2011). Reinventing Medicaid: State innovations to qualify and pay for Patient-Centered Medical Homes show promising results. Health Affairs, 30(7):1325-34. 
The Commonwealth Fund. (2012). Rising to the challenge: Results from a scorecard on local health systems performance. Retrieved from (http://www.commonwealthfund.org) on June 20, 2012.

Thomas, J.W., Grazier, K.L., \& Ward, K. (2004). Economic profiling of primary care physicians: Consistency among risk-adjusted measures. Health Services Research, 39(4):985-1003.

Trapp, D. O. U. G. (2010). Medical homes for Medicaid: The North Carolina Model. American Medical News. Retrieved on August 2, 2015.

van Doorslear, E., Koolman, X., \& Jones, A.M. (2004). Explaining income-related inequalities in doctor utilization in Europe. Health Economics, 13:629-47.

Vanselow, N.A., Donaldson, M.S., \& Yordy, K. S. (1995). A new definition of primary care. Journal of American Medical Association, 273(3):192.

Vargas, R.B., Mangione, C.M, Asch, S., Keesey, J., Mayde, R., Schonlau, M., \& Keeler, E.B. (2007). Can a chronic care model collaborative reduce heart disease risk in patients with diabetes? Society of General Internal Medicine, $22: 215-22$.

Vermont Department of Health. (2008). Blueprint for Health: smart choices, powerful tools, Annual Report 2008. Retrieved from (http://www.healthvermont.gov/admin/legialature/documents/BlueprintAnnua 1Report0109.pdf) on March 15, 2011.

Villagara, V.G., \& Ahmed, T. (2004). Effectiveness of a disease management program for patients with diabetes. Health Affairs, 23:255-266.

Vogel, R.L., \& Ackermann, R.J. (1998). Is primary care physician supply correlated with health outcomes? International Journal of Health Services, 28:183-96.

Vogelli, C., Shields, E.A., Lee, T.A., Gibson, T.B., Marder, W.D., Weiss, K.B., \& Blumenthal, D. (2007). Multiple chronic conditions: Prevalence, health consequences, and implications for quality, care management and costs. Society of General Internal Medicine, 22(3):391-95. 
Wagner, E.H., Austin, B.T., Davis, C., Hindmarsh, M., Schaefer, J., \& Bonomi, A. (2001). Improving chronic illness care: Translating evidence into action. Health Affairs, 20(6):64-78.

Wagner, E.H., Sandhu, N., Newton, K.M., McCulloch, D.K., Ramsey, S.D., \& Grothaus, L.C. (2001). Effects of improved glycemic control on health care costs and utilization. Journal of American Medical Association, 285(2):18289.

Wallace, N.T., McConnell, J.K., Gallia, C.A., \& Smith, J.A (2008) How effective are copayments in reducing expenditures for low-income adult Medicaid beneficiaries? Experience from the Oregon Health Plan. Health Services Research, 43(2):515-30.

Weinberger, M., Oddone, E.Z., \& Henderson, W.G. (1996). Does increased access to primary care reduce hospital readmissions? Veterans Affairs cooperative study group on primary care and hospital readmissions. New England Journal of Medicine, 334:1441-47.

Weinstein, M.C., \& Stason, W.B. (1976). Hypertension: A policy perspective. Harvard University Press, Cambridge, MA

Weissman, J.S., Gatsonis, C., \& Epstein, A.M. (1992). Rates of avoidable hospitalization by insurance status in Massachusetts and Maryland. Journal of American Medical Association, 268(17): 2388-94.

Werner, R.M., Duggan, M., Duey, K., Zhu, J., \&Stuart, E.A. (2013). The PatientCentered Medical Home: An evaluation of a single private payer demonstration in New Jersey. Medical Care, 51(6):487-93.

Westfield, S.A., \& Winkelman, R. A. (2011). Risk adjustment under reform: 2011 health Meeting, Society of Actuaries. Retrieved from (http://www.soa.org) on June 20, 2015.

White, H (1980) A heteroskedasticity-consistent covariance matrix estimator and a direct test for heteroskedasticity. Econometrica, 48(4):817-38. 
Williamson, O.E. (1979). Transaction cost economics: The governance of contractual relations. Journal of Law and Economics, 22(2):233-61.

Williamson, O.E. (2005). The economics of governance. American Economic Review, 95(2):1-18.

Winkelman, R., \& Mehmud, S. (2007). A comparative analysis of claims-based tools for health risk assessment. Society of Actuaries. Retrieved from (http://www.soa.org) on July10, 2013.

Winkelman, R.A \& Damler, R. (2008). Risk adjustment in state Medicaid programs. Health Watch \#57, Society of Actuaries.

Winkelman, R. A., Peper, J., Holland, P., Mehmud, S., \& Woolman, J. (2011). Analysis of HHS proposed rules on reinsurance, risk corridors and risk adjustment. State Health Reform Assistance Network. Retrieved from (http://www.rwjf.org) on May 20, 2013.

Wolff, J. L., Starfield, B., \& Anderson, G. (2002). Prevalence, expenditures, and complications of multiple chronic conditions in the elderly. Archives of Internal Medicine, 162(20):2269-76.

Wu, S.Y., \& Green, A. (2000). Projections of chronic illness prevalence and cost inflation. RAND, Santa Monica, CA.

Yoon, J., Liu, C. F., Lo, J., Schectman, G., Stark, R., Rubenstein, L. V., \& Yano, E. M. (2015). Early changes in VA medical home components and utilization. The American Journal of Managed Care, 21(3):197-204.

Zaslavsky, A.M., \& Epstein, A.M. (2005). How patients' socio-demographic characteristics affect comparisons of competing health plans in California on HEDIS quality measures. International Journal on Quality in Health Care, 17 (1):67-74.

Zhan, C., Miller, M.R., Wong, H., \& Meyers, G.S. (2004). The effects of HMO penetration on preventable hospitalizations. Health Services Research, 39:345-61. 
Zimmerman, B.J., Lindberg, C., \& Plesk, P.E. (1998). Edge ware: Insights from complexity science for health care leaders, Dallas, TX: VHA Publishing. 